University of Tennessee Health Science Center

UTHSC Digital Commons

$12-2011$

\title{
Preclinical Pharmacology of the MDM2 Antagonist Nutlin-3a
}

Fan Zhang

University of Tennessee Health Science Center

Follow this and additional works at: https://dc.uthsc.edu/dissertations

Part of the Medicinal and Pharmaceutical Chemistry Commons, and the Pharmaceutics and Drug Design Commons

\section{Recommended Citation}

Zhang, Fan , "Preclinical Pharmacology of the MDM2 Antagonist Nutlin-3a" (2011). Theses and Dissertations (ETD). Paper 312. http://dx.doi.org/10.21007/etd.cghs.2011.0372.

This Dissertation is brought to you for free and open access by the College of Graduate Health Sciences at UTHSC Digital Commons. It has been accepted for inclusion in Theses and Dissertations (ETD) by an authorized administrator of UTHSC Digital Commons. For more information, please contact jwelch30@uthsc.edu. 


\title{
Preclinical Pharmacology of the MDM2 Antagonist Nutlin-3a
}

\begin{abstract}
Nutlin-3a is an MDM2-p53 interaction antagonist that is under investigation in preclinical models for a variety of pediatric malignancies, including neuroblastoma, retinoblastoma, leukemia, and rhabdomyosarcoma. In the current research, we conducted preclinical pharmacology studies of nutlin-3a to evaluate the synergistic effect of the nutlin-3a and topotecan combination on neuroblastoma cell growth, to assess the effect of nutlin-3a on breast cancer resistance protein (BCRP), and to characterize the disposition of nutlin-3a in the mouse plasma and multiple tissues.
\end{abstract}

Activating the p53 pathway might offer a new therapy for neuroblastoma. In the first part of the study, we assessed the effect of nutlin-3a on the cell viability of neuroblastoma both as a single agent and in combination with topotecan. We showed that targeting MDM2-p53 interaction using nutlin-3a reduced cell growth in neuroblastoma cells. p53 wild-type cells were much more sensitive to nutlin-3a treatment compared to p53 mutant cells. When nutlin-3a was combined with topotecan, a synergistic effect on neuroblastoma cell growth was observed. To explore the mechanism of synergy, we performed quantitative real-time polymerase chain reaction (qRT-PCR) and western blot analysis and found reduction of P-gp expression at both the message level and protein level in p53 wild-type neuroblastoma cells. This is the first study showing the synergistic effect of nutlin-3a in combination with topotecan in neuroblastoma cells and the reduction of P-gp expression by nutlin-3a in p53 wild-type cells.

Although nutlin-3a is currently under pre-clinical investigation as a p53 reactivation agent, it has been recently demonstrated also to have p53 independent actions in cancer cells. In the second part of the study, we first reported that nutlin-3a can inhibit the efflux function of BCRP. We observed that although the nutlin-3a IC50 did not differ between BCRP over-expressing and vector control cells, nutlin-3a treatment significantly potentiated the cells to treatment with the BCRP substrate mitoxantrone. Combination index calculations suggested synergism between nutlin-3a and mitoxantrone in cell lines over-expressing BCRP. Upon further investigation, it was confirmed that nutlin-3a increased the intracellular accumulation of BCRP substrates such as mitoxantrone and Hoechst 33342 in cells expressing functional BCRP without altering the expression level or localization of BCRP. Interestingly, nutlin-3b, considered virtually "inactive" in disrupting the MDM2/p53 interaction, reversed Hoechst 33342 efflux with the same potency as nutlin-3a. Intracellular accumulation and bi-directional transport studies using MDCKII cells suggested that nutlin-3a is not a substrate of BCRP. Additionally, an ATPase assay using Sf9 insect cell membranes over-expressing wild-type BCRP indicated that nutlin-3a inhibits BCRP ATPase activity in a dose-dependent fashion. In conclusion, our studies demonstrate that nutlin-3a inhibits BCRP efflux function, which consequently reverses BCRP-related drug resistance.

Understanding drug disposition is critical in preclinical drug development. In the third part of the study, we used physiologically-based pharmacokinetic (PBPK) modeling to characterize the disposition of nutlin-3a in mice. Plasma protein binding and blood partitioning were assessed by in vitro studies. After intravenous (10 and $20 \mathrm{mg} / \mathrm{kg}$ ) and oral $(50,100$, and $200 \mathrm{mg} / \mathrm{kg}$ ) dosing, tissue concentrations of nutlin-3a were determined in plasma, liver, spleen, intestine, muscle, lung, adipose, bone marrow, adrenal gland, brain, retina, and vitreous fluid. The PBPK model was simultaneously fit to all pharmacokinetic data using NONMEM. Nutlin-3a exhibited nonlinear binding to murine plasma proteins, with the unbound fraction ranging from 0.7 to $11.8 \%$. Nutlin-3a disposition was characterized by rapid absorption with peak plasma concentrations at approximately $2 \mathrm{~h}$ and biphasic elimination consistent with a saturable clearance process. The final PBPK model successfully described the plasma and tissue disposition of nutlin-3a. Simulations suggested high bioavailability, rapid attainment of steady state, and little accumulation when administered once or twice daily at dosages up to $400 \mathrm{mg} / \mathrm{kg}$. The final model was used to perform simulations of unbound tissue concentrations to determine which dosing regimens are 
appropriate for preclinical models of several pediatric malignancies.

In conclusion, our results showed that nutlin-3a synergistically inhibited the growth of neuroblastoma cells when combined with topotecan. Nutlin-3a reversed BCRP-mediated drug resistance by inhibiting the function of BCRP. A PBPK model was successfully established to describe the disposition of nutlin-3a in plasma and tissues of interest for pediatric malignancies.

\section{Document Type}

Dissertation

\section{Degree Name}

Doctor of Philosophy (PhD)

\section{Program}

Pharmaceutical Sciences

\section{Research Advisor}

Clinton F. Stewart, Pharm.D.

\section{Keywords}

ABC transporter, BCRP, Nutlin-3a, Pharmacokinetics, PBPK

\section{Subject Categories}

Medicinal and Pharmaceutical Chemistry | Medicine and Health Sciences | Pharmaceutics and Drug Design | Pharmacy and Pharmaceutical Sciences 


\title{
PRECLINICAL PHARMACOLOGY OF THE MDM2 ANTAGONIST NUTLIN-3A
}

\author{
A Dissertation \\ Presented for \\ The Graduate Studies Council \\ The University of Tennessee \\ Health Science Center
}

\author{
In Partial Fulfillment \\ Of the Requirements for the Degree \\ Doctor of Philosophy \\ From The University of Tennessee
}

By

Fan Zhang

December 2011 
Chapter 2 (C) 2011 by Elsevier.

Chapter 3 (C) 2011 by American Society for Pharmacology and Experimental Therapeutics.

All other material (C) 2011 by Fan Zhang.

All rights reserved. 


\section{DEDICATION}

I dedicate this dissertation to my parents, Hua Zhang and Yufang Zan, and to my husband and son, Fei Ma and Alexander Zhang Ma. 


\section{ACKNOWLEDGEMENTS}

I would like to take this opportunity to express my deepest gratitude to all those who supported and helped me during my research. First and foremost, I would like to thank my mentor, Dr. Clinton F. Stewart, for his guidance, support, encouragement, and inspiration. I am incredibly lucky to be able to be a student of Dr. Stewart and to learn from his wisdom. I sincerely appreciate Dr. Stewart for everything that he has taught me.

My gratitude is also extended to the other members of my dissertation committee: Drs. R. Kiplin Guy, Bernd Meibohm, Victor M. Santana, Michael A. Dyer, and former member Dr. Richard T. Williams for serving on my committee and providing excellent feedback and guidance throughout this project. I also would like to thank Guy laboratory for providing the nutlin-3a used in this study.

In addition, I want to thank every member and friend in the Stewart laboratory. Thanks very much to our lab supervisor Dr. Stacy Throm, former supervisor Laura Miller, and co-workers Dr. Laura Lea Murley, Dr. Michael Tagen, Dr. John Panetta, Dr. Fan Wang, Zaifang Huang, Dr. Feng Bai, Mohamed Elmeliegy, Dr. Steven Zatechka, Thandranese Owens, Jenkin Chen, Daniel Groepper, and Rachel Kennedy for their help throughout the project. In addition, I would also like to thank Jeremy Mallari, Katie Nemeth, Fangyi Zhu, Jiakun Zhang, Min Lu, Nidal Boulos, Lei Yang, Shelly Wilkerson, and Frederique Zindy for their help in the nutlin-3a pharmacokinetic study.

Further, I would like to thank the faculty members and friends in the Department of Pharmaceutical Sciences at St. Jude Children's Research Hospital and the University of Tennessee Health Science Center.

Finally, I extend deep thanks to my parents, my husband, and my parents -in-law for their love and support. 


\begin{abstract}
Nutlin-3a is an MDM2-p53 interaction antagonist that is under investigation in preclinical models for a variety of pediatric malignancies, including neuroblastoma, retinoblastoma, leukemia, and rhabdomyosarcoma. In the current research, we conducted preclinical pharmacology studies of nutlin-3a to evaluate the synergistic effect of the nutlin-3a and topotecan combination on neuroblastoma cell growth, to assess the effect of nutlin-3a on breast cancer resistance protein (BCRP), and to characterize the disposition of nutlin-3a in the mouse plasma and multiple tissues.
\end{abstract}

Activating the p53 pathway might offer a new therapy for neuroblastoma. In the first part of the study, we assessed the effect of nutlin-3a on the cell viability of neuroblastoma both as a single agent and in combination with topotecan. We showed that targeting MDM2-p53 interaction using nutlin-3a reduced cell growth in neuroblastoma cells. p53 wild-type cells were much more sensitive to nutlin-3a treatment compared to p53 mutant cells. When nutlin-3a was combined with topotecan, a synergistic effect on neuroblastoma cell growth was observed. To explore the mechanism of synergy, we performed quantitative real-time polymerase chain reaction (qRT-PCR) and western blot analysis and found reduction of P-gp expression at both the message level and protein level in p53 wild-type neuroblastoma cells. This is the first study showing the synergistic effect of nutlin-3a in combination with topotecan in neuroblastoma cells and the reduction of P-gp expression by nutlin-3a in p53 wild-type cells.

Although nutlin-3a is currently under pre-clinical investigation as a p53 reactivation agent, it has been recently demonstrated also to have p53 independent actions in cancer cells. In the second part of the study, we first reported that nutlin-3a can inhibit the efflux function of BCRP. We observed that although the nutlin-3a $\mathrm{IC}_{50}$ did not differ between BCRP over-expressing and vector control cells, nutlin-3a treatment significantly potentiated the cells to treatment with the BCRP substrate mitoxantrone. Combination index calculations suggested synergism between nutlin-3a and mitoxantrone in cell lines over-expressing BCRP. Upon further investigation, it was confirmed that nutlin-3a increased the intracellular accumulation of BCRP substrates such as mitoxantrone and Hoechst 33342 in cells expressing functional BCRP without altering the expression level or localization of BCRP. Interestingly, nutlin-3b, considered virtually "inactive" in disrupting the MDM2/p53 interaction, reversed Hoechst 33342 efflux with the same potency as nutlin-3a. Intracellular accumulation and bi-directional transport studies using MDCKII cells suggested that nutlin-3a is not a substrate of BCRP. Additionally, an ATPase assay using Sf9 insect cell membranes over-expressing wild-type BCRP indicated that nutlin-3a inhibits BCRP ATPase activity in a dose-dependent fashion. In conclusion, our studies demonstrate that nutlin-3a inhibits BCRP efflux function, which consequently reverses BCRP-related drug resistance.

Understanding drug disposition is critical in preclinical drug development. In the third part of the study, we used physiologically-based pharmacokinetic (PBPK) modeling to characterize the disposition of nutlin-3a in mice. Plasma protein binding and blood 
partitioning were assessed by in vitro studies. After intravenous (10 and $20 \mathrm{mg} / \mathrm{kg}$ ) and oral $(50,100$, and $200 \mathrm{mg} / \mathrm{kg})$ dosing, tissue concentrations of nutlin-3a were determined in plasma, liver, spleen, intestine, muscle, lung, adipose, bone marrow, adrenal gland, brain, retina, and vitreous fluid. The PBPK model was simultaneously fit to all pharmacokinetic data using NONMEM. Nutlin-3a exhibited nonlinear binding to murine plasma proteins, with the unbound fraction ranging from 0.7 to $11.8 \%$. Nutlin-3a disposition was characterized by rapid absorption with peak plasma concentrations at approximately $2 \mathrm{~h}$ and biphasic elimination consistent with a saturable clearance process. The final PBPK model successfully described the plasma and tissue disposition of nutlin-3a. Simulations suggested high bioavailability, rapid attainment of steady state, and little accumulation when administered once or twice daily at dosages up to 400 $\mathrm{mg} / \mathrm{kg}$. The final model was used to perform simulations of unbound tissue concentrations to determine which dosing regimens are appropriate for preclinical models of several pediatric malignancies.

In conclusion, our results showed that nutlin-3a synergistically inhibited the growth of neuroblastoma cells when combined with topotecan. Nutlin-3a reversed BCRP-mediated drug resistance by inhibiting the function of BCRP. A PBPK model was successfully established to describe the disposition of nutlin-3a in plasma and tissues of interest for pediatric malignancies. 


\section{TABLE OF CONTENTS}

CHAPTER 1. INTRODUCTION .....................................................................................1

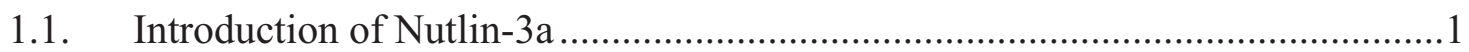

1.1.1. Restoring p53 as a therapeutic strategy ...............................................

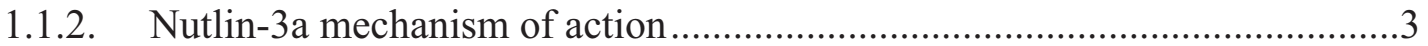

1.1.3. Reactivation of the p53 pathway by nutlin-3a in vitro ...............................

1.1.4. In vivo anti-tumor effect of nutlin-3a.....................................................

1.2. ABC Transporters and Drug Interaction.....................................................

1.2.1. ABC transporter family.......................................................................

1.2.2. Role of ABC transporter in drug ADME ................................................9

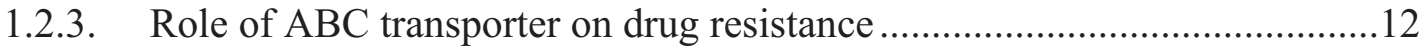

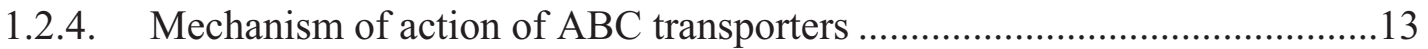

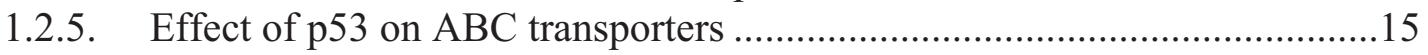

1.2.6. Evaluation of transporter mediated drug-drug interaction..........................16

1.3. Physiologically-based Pharmacokinetic (PBPK) Modeling and Simulation

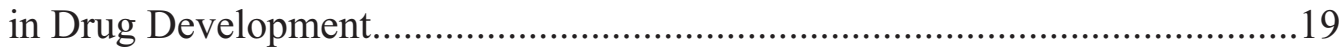

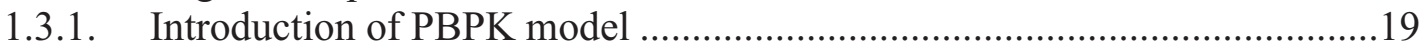

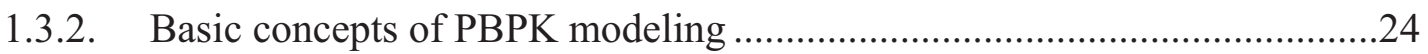

1.3.3. Applications of PBPK model in drug discovery and development .............29

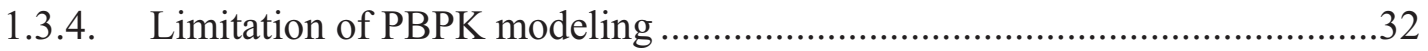

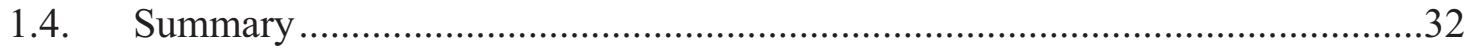

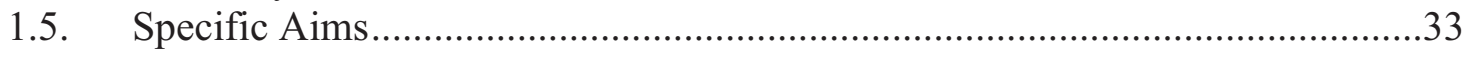

\section{CHAPTER 2. MDM2 ANTAGONIST NUTLIN-3A SYNERGISTICALLY INHIBITS NEUROBLASTOMA CELL GROWTH WITH TOPOTECAN ...........34}

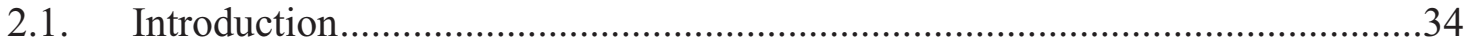

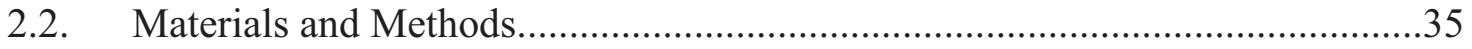

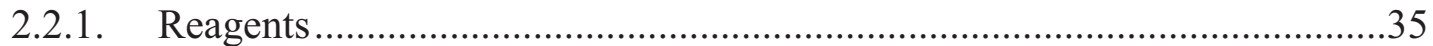

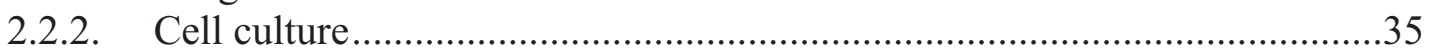

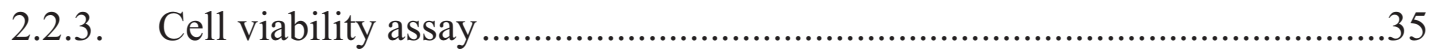

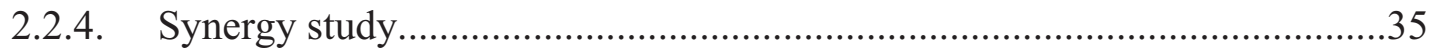

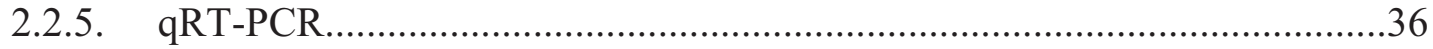

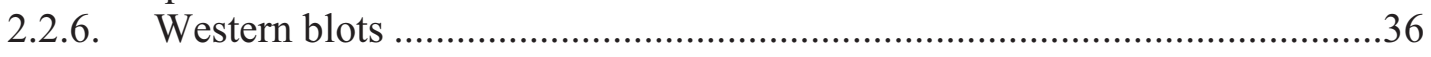

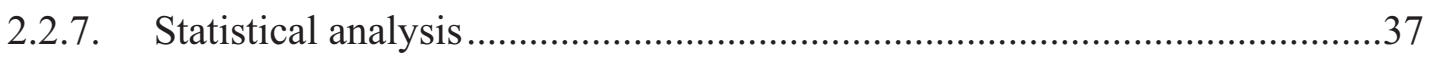

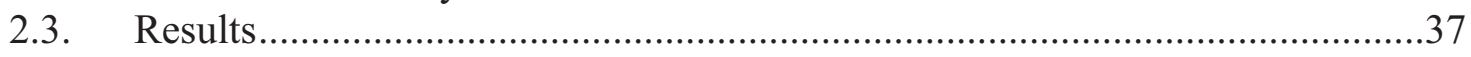

2.3.1. Effect of single agent nutlin-3a on the cell viability of neuroblastoma

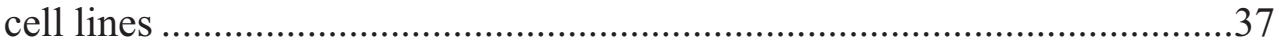

2.3.2. Combination of nutlin-3a with topotecan in NB1691 cells .........................37

2.3.3. Synergistic effect of nutlin-3a in combination with topotecan .....................37

2.3.4. MDRl gene expression change after nutlin-3a treatment ...........................41

2.3.5. $\quad$ P-gp protein expression after nutlin-3a treatment.....................................4

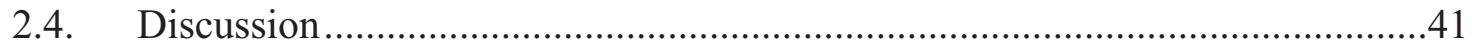




\section{CHAPTER 3. MDM2 ANTAGONIST NUTLIN-3A REVERSES MITOXANTRONE RESISTANCE BY INHIBITING BREAST CANCER RESISTANCE PROTEIN MEDIATED DRUG TRANSPORT}

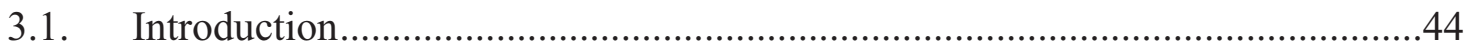

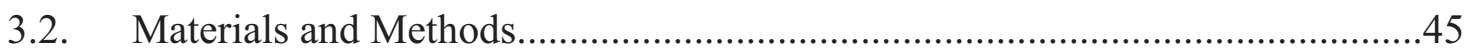

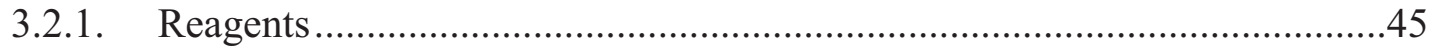

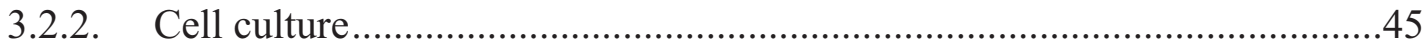

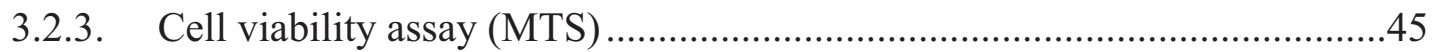

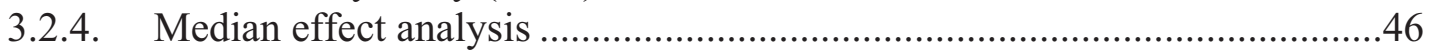

3.2.5. Intracellular accumulation and efflux of mitoxantrone by confocal

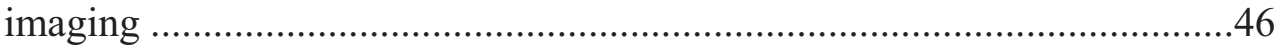

3.2.6. Hoechst 33342 dye accumulation and efflux studies by flow cytometry ....46

3.2.7. Intracellular accumulation and efflux of Hoechst 33342 by widefield

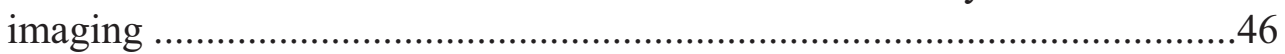

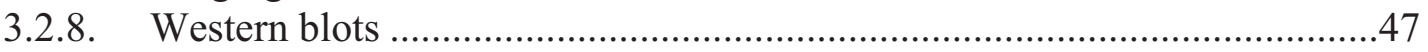

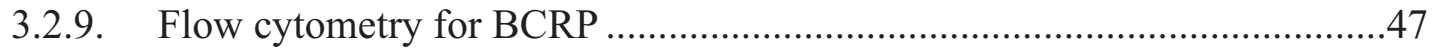

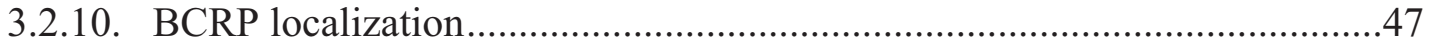

3.2.11. Intracellular accumulation of nutlin-3a.................................................48

3.2.12. Bi-directional transport across MDCKII monolayer cells ..........................48

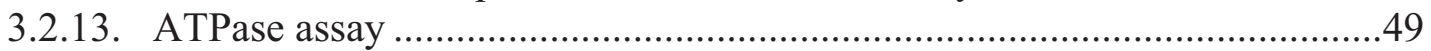

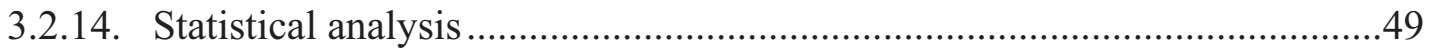

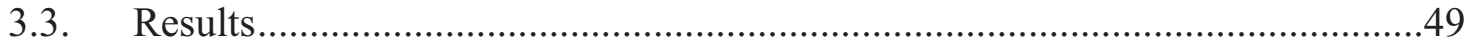

3.3.1. Nutlin-3a sensitizes BCRP expressing cells to mitoxantrone treatment......49

3.3.2. Nutlin-3a inhibits BCRP-mediated transport of mitoxantrone ....................50

3.3.3. Nutlin-3a inhibits BCRP-mediated transport of Hoechst 33342 ..................55

3.3.4. Nutlin-3a treatment does not alter BCRP expression or localization ..........55

3.3.5. Nutlin-3a is not a substrate for BCRP.......................................................63

3.3.6. Nutlin-3a inhibits the ATPase activity of BCRP .....................................63

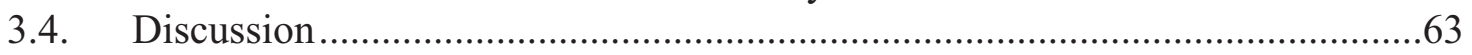

\section{CHAPTER 4. WHOLE-BODY PHYSIOLOGICALLY BASED}

PHARMACOKINETIC MODEL FOR NUTLIN-3A IN MICE AFTER INTRAVENOUS AND ORAL ADMINISTRATION ..........................................69

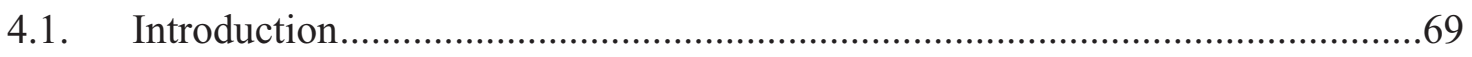

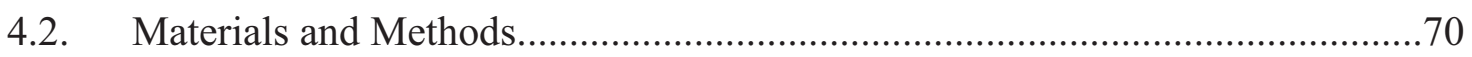

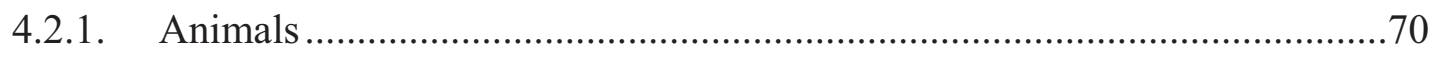

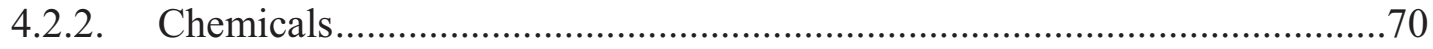

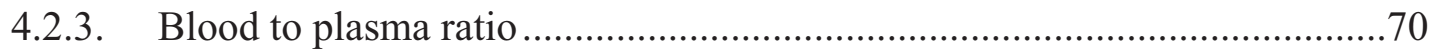

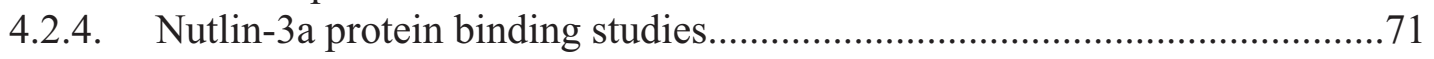

4.2.5. Drug administration and sample collection ........................................... 71

4.2.6. Quantitative analysis of nutlin-3a ..................................................... 72

4.2.7. Whole body PBPK model development .............................................. 72

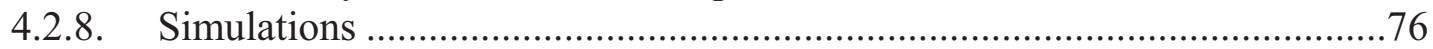

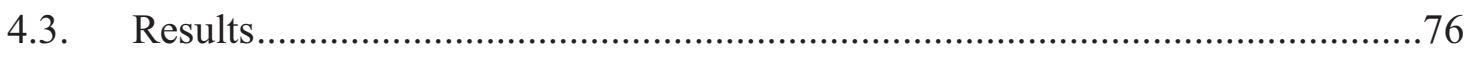

4.3.1. Blood to plasma partitioning and plasma protein binding of nutlin-3a .......76

4.3.2. Nutlin-3a pharmacokinetics in mice ................................................... 78 
4.3.3. Application of PBPK model to the design of nutlin-3a dosing regimens

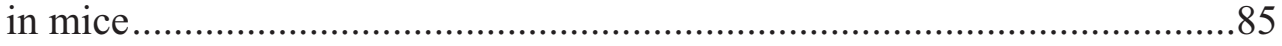

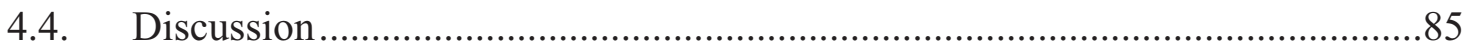

CHAPTER 5. SUMMARY AND FUTURE DIRECTIONS .......................................93

LIST OF REFERENCES ...........................................................................................97

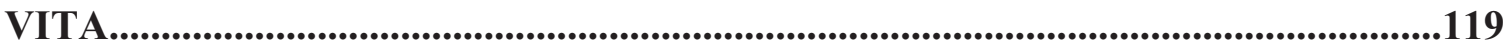




\section{LIST OF TABLES}

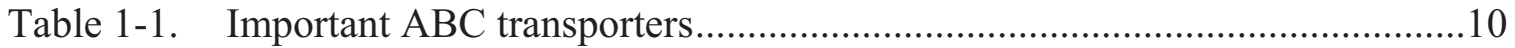

Table 2-1. Combination of nutlin-3a with topotecan in NB1691 cells ..........................38

Table 2-2. Synergistic effect of nutlin-3a in combination with topotecan....................39

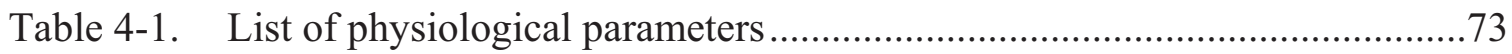

Table 4-2. $\quad$ Estimated PBPK model parameters ......................................................82

Table 4-3. Nutlin-3a IC $_{50}$ for cell survival in different cell types ..............................86

Table 4-4. Percent time unbound tissue concentration is above unbound $\mathrm{IC}_{50} \ldots \ldots \ldots \ldots . . . .87$ 


\section{LIST OF FIGURES}

Figure 1-1. p53 pathway and MDM2/MDMX p53 interaction ....................................2

Figure 1-2. Chemical structure of nutlin-3 .........................................................4

Figure 1-3. Nutlin-3a binds to the MDM2-p53 and MDMX-p53 binding pockets.........4

Figure 1-4. The structures of three categories of ABC transporter .............................

Figure 1-5. Mechanism of action of a typical ABC transporter ................................... 14

Figure 1-6. Decision trees for studying transporter-based drug-drug interactions .........17

Figure 1-7. Example model structure of the classical PK model ...............................21

Figure 1-8. Typical structure of a whole-body PBPK model ....................................22

Figure 1-9. Structures and equations for individual organ models.............................25

Figure 1-10. Schematic structure of open loop and closed loop.................................28

Figure 1-11. General schemes to incorporate drug dependent parameters into a PBPK

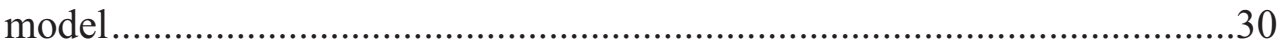

Figure 2-1. Effect of single agent nutlin-3a on the cell viability of neuroblastoma

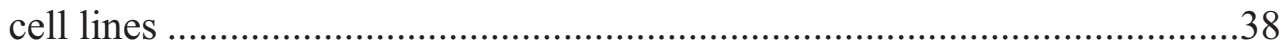

Figure 2-2. $\quad M D R 1$ gene expression change after nutlin-3a treatment ........................42

Figure 2-3. P-gp protein expression change after nutlin-3a treatment ........................42

Figure 3-1. BCRP expression does not confer resistance to nutlin-3a treatment ...........51

Figure 3-2. Co-treatment of cells with nutlin-3a and mitoxantrone strongly reverses BCRP mediated drug resistance to mitoxantrone .....................................52

Figure 3-3. Synergistic effects of nutlin-3a in combination with mitoxantrone.............53

Figure 3-4. Nutlin-3 treatment strongly increases the intracellular accumulation of mitoxantrone in Saos-2-BCRP cell lines.

Figure 3-5. Nutlin-3a dose-dependently inhibits BCRP efflux of Hoechst 33342,

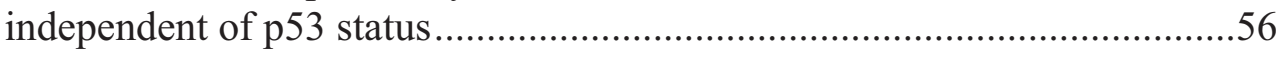

Figure 3-6. Effect of FTC on the BCRP efflux of Hoechst 33342 .............................57

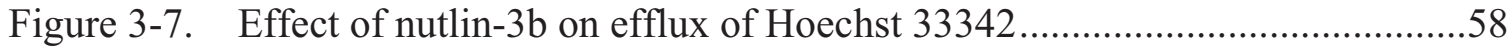


Figure 3-8. Viability ratio of Saos-2-BCRP/ Saos-2-pcDNA3.1 during the Hoechst 33342 efflux study.

Figure 3-9. Nutlin-3 treatment increases the intracellular accumulation of Hoechst 33342 in Saos-2-BCRP cell lines

Figure 3-10. Nutlin-3a does not alter levels of BCRP protein expression

Figure 3-11. Nutlin-3a does not alter cellular localization of BCRP in Saos-2 cells

Figure 3-12. No difference in intracellular accumulation of nutlin-3a in the presence of BCRP

Figure 3-13. Trans-epithelial transport of nutlin-3a $(10 \mu \mathrm{M})$ in MDCKII-pcDNA3.1 and MDCKII-BCRP cells indicates nutlin-3a is not a substrate of BCRP ..65

Figure 3-14. Nutlin-3a dose dependently inhibited BCRP ATPase activity .66

Figure 4-1. Schematic diagram of PBPK model for nutlin-3a in mice .74

Figure 4-2. Analysis of nutlin-3a characteristics in murine blood .77

Figure 4-3. Nutlin-3a binding to murine plasma proteins .79

Figure 4-4. Comparison of actual plasma and tissue concentrations of nutlin-3a..........80

Figure 4-5. Concentration-time plots of nutlin-3a in tissues . .81

Figure 4-6. Simulated concentration-time plot of plasma nutlin-3a after multiple oral doses with once-daily (QD) and twice-daily (BID) dosing.....

Figure 4-7. Plasma area under the concentration-time curve for $24 \mathrm{~h}$ at steady state (AUC0-24) versus nutlin-3a dosage when administered once daily (QD) and twice daily (BID).

Figure 4-8. Nutlin-3a binding to cell culture media . .86

Figure 4-9. Simulated concentration-time plot of unbound nutlin-3a in the retina and vitreous after multiple oral doses given once daily (QD) or twice daily (BID)

Figure 4-10. Simulated concentration-time plot of unbound nutlin-3a in the adrenal gland after multiple oral doses given once daily (QD) or twice daily (BID)

Figure 4-11. Simulated concentration-time plot of unbound nutlin-3a in the muscle after multiple oral doses given once daily (QD) or twice daily (BID). .88 
Figure 4-12. Simulated concentration-time plot of unbound nutlin-3a in the plasma, bone marrow, and spleen after multiple oral doses given once daily

(QD) or twice daily (BID)

Figure 5-1. Nutlin-3a inhibits the activity of CYP3A4 in vitro...................................94 


\section{LIST OF ABBREVIATIONS}

$\mathrm{ABC}$

ADME

ADP

AIC

ALL

AML

ATP

AUC

$\mathrm{BBB}$

BCEC

BCRP

BCS

BDDCS

CI

CL

CNS

$\mathrm{CSF}$

DDI

DMEM

DMSO

$\mathrm{Fa}$

FBS

FDA

$\mathrm{Fg}$

GST

HBSS

IND

LLOQ

MDCK

MDM2

MDR

MRP1

MXR

NB

NBD

NDA

NME

NSCLC
ATP-binding cassette

Adsorption, distribution, metabolism, and excretion

Adenosine diphosphate

Akaike's information criterion

Acute lymphoblastic leukemia

Acute myelogenous leukemia

Triphosphate

Area under the curve

Blood-brain barrier

Brain capillary endothelial cells

Breast cancer resistance protein

Biopharmaceutics classification system

Biopharmaceutics drug dispositionclassification system

Combination index

Clearance

Central nervous system

Cerebrospinal fluid

Drug-drug interaction

Dulbecco's modified eagle's medium

Dimethyl sulfoxide

Fraction of absorption

Fetal bovine serum

Food and drug administration

Fraction of intact drug escaping gut metabolism

Glutathione S-transferase

Hank's balance salt solution

Investigational new drugs

Lower limit of quantification

Madin-Darby canine kidney epithelial

Murine double minute-2

Multiple drug resistance

Multidrug resistance protein 1

Mitoxantrone resistance protein

Neuroblastoma

Nucleotide binding domain

New drug applications

New molecular entity

Non-small cell lung cancer 
OAT

OATP

OCT

PBPK

P-gp

PhRMA

Pi

PI

P-PBPK

PXR

QRT-PCR

SLC

SP

SULT

TMD

UGT

17-AAG
Organic anion transporter

Organic anion-transporting polypeptide

Organic cation transporter

Physiologically based pharmacokinetic

P-glycoprotein

Pharmaceutical research and manufacturers of America

Inorganic phosphate

Propidium iodide

Pediatric PBPK

Pregnane $\mathrm{X}$ receptor

Quantitative real time polymerase chain reaction

Solute carrier

Side population

Sulfotransferase

Transmembrane domain

Glucuronosyltransferases

17-(allylamino)-17-demethoxygeldanamycin 


\section{CHAPTER 1. INTRODUCTION}

\subsection{Introduction of Nutlin-3a}

\subsubsection{Restoring p53 as a therapeutic strategy}

Studies from the past three decades show tumor suppressor protein p53 plays important roles in organizing cell defense against cancerous transformation. In responses to stress conditions such as irradiation, DNA damages, and hypoxia, p53 works as a potent transcription factor that activates downstream genes, leading to cell cycle arrest, apoptosis, and inhibition of angiogenesis [1]. p53 can also exert a pro-apoptotic function independent of transcriptional functions $[2,3]$.

Tumor suppressor p53 is mutated in half of the human tumors [4]. In those tumors that retain wild-type p53, p53 is tightly regulated and maintained at low or undetectable levels. p53 protein has a short half-life of $\sim 20$ minutes [5] due to the key negative regulator oncogene murine double minute-2 (MDM2). MDM2 (also known as HDM2) acts as an E3 ubiquitin ligase that facilitates the export of p53 from the nucleus to the cytoplasm and targets p53 for ubiquitin-dependent proteasome degradation $[6,7]$. In addition, MDM2 inhibits 553 function by direct binding to the transcriptional binding site of $\mathrm{p} 53$, thereby preventing its interaction with the transcription machinery [8]. p53 and MDM2 interact to form an auto-regulatory loop, where increased p53 transcriptionally activates MDM2 and the latter in turn decreases the level of p53 [9] (Figure 1-1). MDMX (also known as a MDM4 or HDMX), sharing substantial structural homology with MDM2, also has an important role in regulating p53 [10-12]. In addition to inhibiting the transcriptional activity of p53, MDMX forms a heterocomplex with MDM2 that potentiates the ubiquitylation and degradation of $\mathrm{p} 53$ [13, 14]. Unlike MDM2, MDMX is not a transcriptional target of p53. Binding of MDMX with MDM2 can cause ubiquitination and degradation of MDMX.

Considering the important roles of MDM2/MDMX in p53 stability and function, restoration of the impaired function of $\mathrm{p} 53$ by inhibiting MDM2/MDMX was considered an attractive strategy to treat tumors with wild-type p53 [15]. Several inhibitors of MDM2/MDMX have been discovered and are currently under investigations. For example, a phase I study for JNJ-26854165 (developed by Johnson \& Johnson, USA) in patients with advanced stage or refractory solid tumors (ClinicalTrials.gov identifier number: NCT00676910) was completed last year. Phase I clinical trials for RO5045337 (RG7112, developed by F. Hoffmann-La Roche, USA) are ongoing for patients with solid tumors (ClinicalTrials.gov identifier numbers: NCT00559533 and NCT01164033), hematologic neoplasms (ClinicalTrials.gov identifier number: NCT00623870), and liposarcomas (ClinicalTrials.gov identifier number: NCT01143740). Other compounds such as MI-219 [16] (also known as AT219), nutlin-3a [17], SJ-172550 [18], and benzodiazepines [19] are in preclinical development stage. 


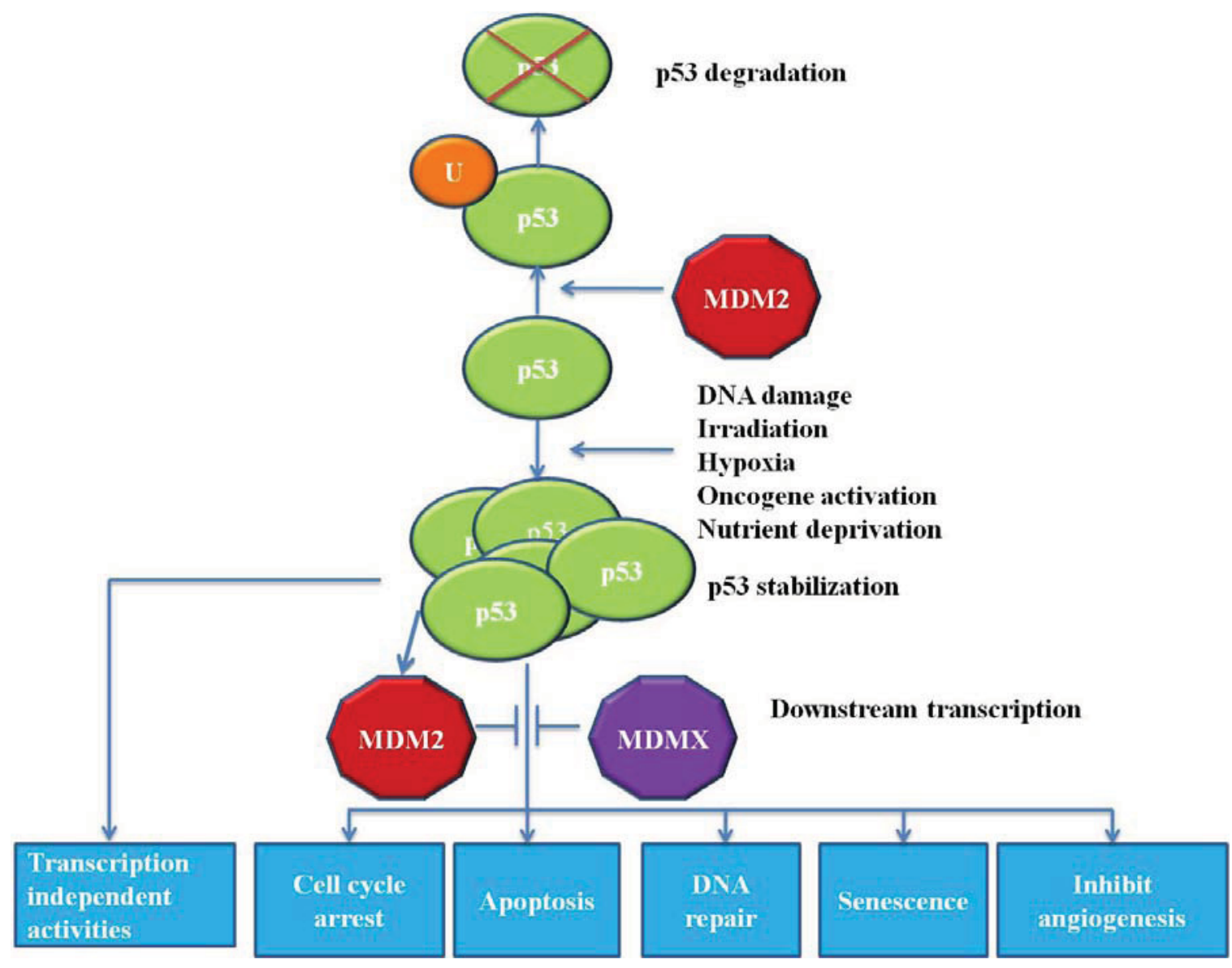

Figure 1-1. p53 pathway and MDM2/MDMX p53 interaction 


\subsubsection{Nutlin-3a mechanism of action}

In 2004, Vassilev and colleagues reported a group of imidazoline compounds called nutlins are able to inhibit MDM2-p53 binding with high binding potency and selectivity [17]. Nutlin-3 (Figure 1-2) is the most potent compound among the three nutlins (nutlin-1, nutlin-2, and nutlin-3). So far, nutlin-3 is the most widely published small molecule inhibitor of MDM2/MDMX-p53 interaction. Nutlin-3 is a racemic mixture of nutlin-3a (active enantiomer) and nutlin-3b (inactive enantiomer). The binding affinity for nutlin-3a to MDM2 is 150-fold higher than nutlin-3b [17].

Successful development of nutlin is based on understanding the structural biology of the p53-MDM2 interaction. Kussie and colleagues reported a relative deep p53 binding pocket on the surface of the MDM2 protein [20]. Specifically, they found that only three amino acid residues (Phe19, Trp23, and Leu26) of p53 are critical to the binding and fit tightly in the MDM2 binding pocket. This finding made de novo synthesis of small molecule inhibitors of the MDM2-p53 interaction possible. Nutlins were generated by combing structure-based screening of the 3D database, high-throughput screening of large chemical libraries, and extensive chemical modifications of the lead compounds. Crystal structure data of MDM2-nutlin complex proved the binding of nutlin to the p53 pocket $[17,21]$. The ethoxy group on the nutlin occupies the position of Phe19, the bromophenyl group occupies the position of Trp23, and the others occupy the position of Leu26 [22]. Since MDM2 and MDMX share structure/sequence similarity and MDMX binds to the similar region of p53 [23], nutlin-3 also binds to MDMX with lower affinity (Figure 1-3) [17, 24].

\subsubsection{Reactivation of the $\mathrm{p53}$ pathway by nutlin-3a in vitro}

Since 2004, many in vitro studies have been conducted to examine the effect of nutlin-3 on cell cycle arrest and apoptosis. For example, the effect of nutlin-3a and nutlin-3b $(1.25-10 \mu \mathrm{M})$ on cell cycle arrest was examined in a panel of cancer cell lines from different tumor types, including colorectal (HCT116 and RKO), lung (H460 and A549), breast (MCF7), prostate (LnCaP and 22Rv1), melanoma (LOX), osteosarcoma (SJSA-1), and renal cancer (A498) [25]. 24 hour treatment of nutlin-3a induced a reduction/depletion of the S-phase fraction, as well as G1 and G2 arrest in all the p53 wild-type cell lines tested. Expression of p21, an essential element of p53-induced cell cycle arrest, increased after nutlin-3a treatment. In contrast, these effects were not observed in inactive enantiomer nutlin-3b treatment groups. Colon cancer cells with mutant p53 (HT29) did not respond to nutlin-3a treatment in vitro and in vivo [25].

In contrast to cell cycle arrest, the pro-apoptotic effect is more variable. Apoptosis after nutlin-3a (or -3b) treatment (24 72 hours) was evaluated by Annexin V assay [25]. Annexin $\mathrm{V}$ positive fractions varied among p53 wild-type cells from as high as $80 \%$ (SJSA-1) to 10\% (A549 and HCT116). Since incubation cells with doxorubicin $(250 \mathrm{nM})$ for $48 \mathrm{~h}$ led to a dramatic increase of the Annexin $\mathrm{V}$-positive cell fraction in all of the tested lines (including the cell line that had low Annexin V-positive faction after nutlin-3 


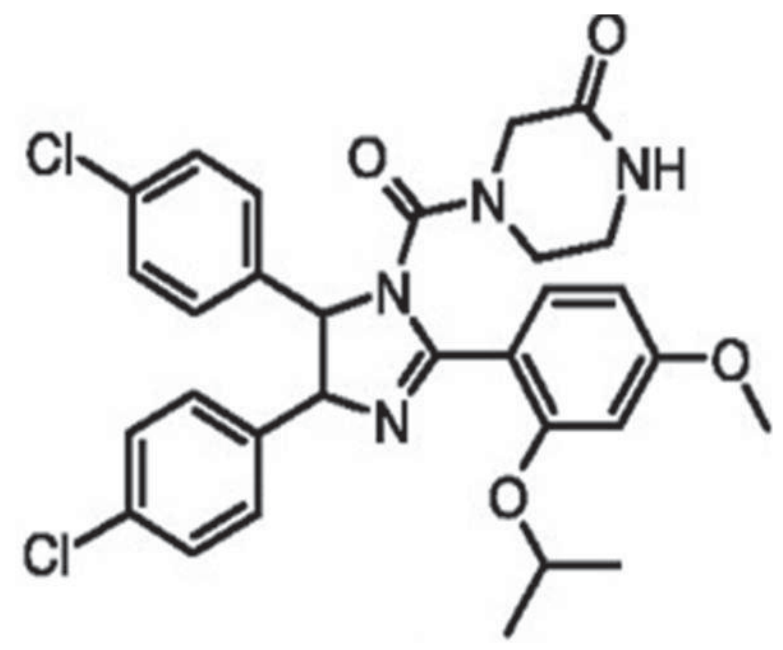

Figure 1-2. Chemical structure of nutlin-3
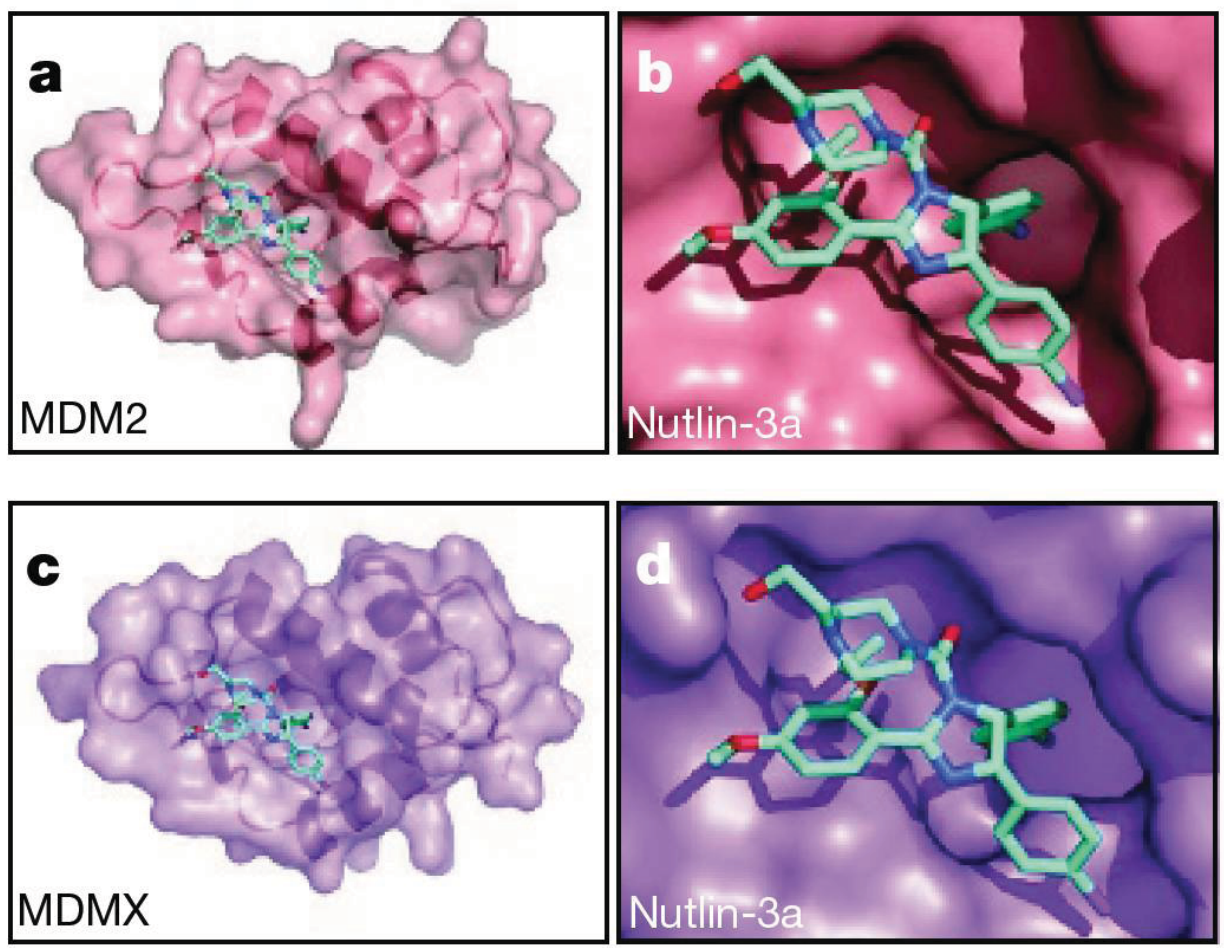

Figure 1-3. Nutlin-3a binds to the MDM2-p53 and MDMX-p53 binding pockets

Source: Reprinted with permission. Laurie, N.A., et al., Inactivation of the $p 53$ pathway in retinoblastoma. Nature, 2006. 444(7115): p. 61-6. 
treatment), the authors concluded that the low apoptotic level observed after nutlin-3 treatment is not caused by defects in the general components of the apoptotic machinery; rather these cells might have defects in p53-dependent apoptotic signaling.

Studies suggested correlations between high MDM2 expression and strong apoptosis response when p53 wild-type cells are treated with nutlin-3. A significant correlation between MDM2 expression levels and sensitivity to nutlin-3 in p53 wild-type cells was observed in 18 ALL cell lines and 30 primary leukemia samples [26]. Nutlin-3 potently killed wild-type p53 ALL cells over-expressing MDM2. Osteosarcoma cells SJSA-1 and MHM, p53 wild-type cells with 25- and 10- fold MDM2 gene amplification and high MDM2 expression, had the strongest apoptosis response among a panel of 10 p53 wild-type cell lines tested by Annexin-V and microarray analysis [25]. LNCaP (prostate cancer), 22Rv1 (prostate cancer), and RKO (colon cancer) cells with a single copy of MDM2 gene had intermediate levels of apoptotic response. HCT-116 (colon cancer) and U20S (osteosarcoma) cell lines, lacking the MDM2 gene amplification, had the lowest apoptosis response. Thus, MDM2 expression in tumors may be a valuable response biomarker in the clinic. However, studies for MDM2 might not directly translate to MDMX. In fact, Hu and colleges reported that MDMX over-expression prevents p53 activation by nutlin-3 [27].

In addition, some other characteristics of nutlin-3a are worth mentioning: 1).Unlike radiation and traditional chemotherapy drugs, nutlin-3 activates p53 in a nongenotoxic manner. 2). Nutlin-3 induces apoptosis in p53 wild-type cancer cells; however, it only causes cell cycle arrest in normal cells, which may help protect normal cells from cytotoxic chemotherapies. Thus nutlin-3 was proposed to act as a chemo-protective agent [28]. 3). Multiple studies have suggested synergistic/sensitizing effects of nutlin-3 with radiation [29] or other chemotherapeutic drugs including (but not limited to) topotecan (in retinoblastoma cells) [24], doxorubicin and selumetinib (in acute myeloid leukemia cells) [30, 31], chlorambucil, doxorubicin, fludarabine, dasatinib (in chronic lymphocytic leukemia cells) [32-35], R-roscovitine (in neuroblastoma cells) [36], vincristine, actinomycin D, doxorubicin, etoposide (in Ewing sarcoma cells), and bortezomib (in myeloma, thyroid, breast, and prostate carcinomas and colon carcinoma cells) $[37,38]$.

\subsubsection{In vivo anti-tumor effect of nutlin-3a}

In vivo, nutlin-3a monotherapy demonstrated anti-tumor efficacy in preclinical models of human osteosarcoma, prostate cancer, retinoblastoma, KSHV lymphoma, and neuroblastoma with wild-type p53 [17, 24, 25, 39-41]. Vassilev et al. first reported the in vivo activity of nutlin-3 in nude mice bearing subcutaneous human osteosarcoma xenograft (SJSA-1). Nutlin-3 (po. 200mg/kg BID for 3 weeks) was well tolerated without causing significant weight loss or any gross abnormalities upon necropsy at the end of the treatment. Compared to the vehicle control group, nutlin-3 treatment resulted in $90 \%$ inhibition of tumor growth [17]. 
Tovar et al. conducted in vivo study of nutlin-3a in nude mice bearing SJSA-1 (osteosarcoma), MHM (osteosarcoma), LNCaP (prostate cancer), 22Rv1 (prostate cancer) and HT29 (colon cancer) tumors [25]. SJSA-1-bearing mice were treated with an oral dose of $50 \mathrm{mg} / \mathrm{kg}, 100 \mathrm{mg} / \mathrm{kg}$ or $200 \mathrm{mg} / \mathrm{kg}$ nutlin-3a twice daily for 3 weeks. Nutlin-3a dose dependently suppressed SJSA-1 tumor growth, with substantial tumor shrinkage observed in the $200 \mathrm{mg} / \mathrm{kg}$ treatment group. The $200 \mathrm{mg} / \mathrm{kg}$ oral nutlin-3a twice daily regimen was also efficacious in MHM (3 weeks treatment), LNCaP (2 weeks treatment), and 22Rv1 (2 weeks treatment) models with average tumor growth inhibition $>98 \%$. In p53 mutant HT29 xenograft, nutlin-3a did not reduce the tumor size. The data showed a reasonable correlation between in vitro and in vivo tumor response. Similar to the report from Vassilev et al., no weight loss or significant pathological changes were observed during the study.

Laurie et al. conducted the first in vivo study to assess the effect of nutlin-3 on retinoblastoma [24]. Subconjunctival injections of $1 \mu 1$ nutlin-3 $(170 \mathrm{mM})$ and topotecan $(2 \mathrm{mM})$ both as a single agent and in combination were administered into each eye of tumor-bearing mice daily for 5 days. Total treatment amount per eye was 85 pmol nutlin-3 and 2 nmol topotecan. Both nutlin-3 and topotecan were effective as a single agent in the Y79-luc orthotopic model. The combination of subconjunctival topotecan and nutlin-3 resulted in an 82-fold tumor burden reduction with no ocular or systemic side-effects. Brennan et al. recently reported a study aimed to identify better chemotherapeutic combinations for the treatment of retinoblastoma in genetically engineered mouse models and orthotopic xenograft models of human retinoblastoma [42]. SCID mice bearing SJ-39 retinoblastoma tumor cells received vincristine/toposide/carboplatin, carboplatin(subconj)/topotecan(syst), or carboplatin(subconj)/topotecan(syst) alternating with nutlin-3a(OC)/topotecan(syst). The nutlin-3a(OC)/topotecan(syst)-containing group showed significantly better response. Subconjunctival administrations of nutlin-3a alone or in combination with topotecan were well tolerated without ocular or systemic toxicity.

Van Maerken et al. reported the effect of nutlin-3 on nude mice bearing chemo-resistant, MYCN-amplified neuroblastoma [43]. $200 \mathrm{mg} / \mathrm{kg}$ oral nutlin-3 twice daily treatment reduced tumor growth and metastasis in the p53 wild-type UKF-NB- ${ }^{3} \mathrm{rDOX}^{20}$ xenograft without causing signs of toxicity. No treatment effect was observed in p53 mutant UKF-NB- $3{ }^{\mathrm{r}} \mathrm{VCR}^{10}$ xenograft, suggesting p53 status significantly influences the in vivo response to nutlin-3 treatment.

\section{2. $\mathrm{ABC}$ Transporters and Drug Interaction}

\subsubsection{ABC transporter family}

Transporters are membrane proteins that play important roles in controlling the influx and efflux of ions, glucose, bile acids, vitamins, hormones, lipids, fatty acids, toxins, and drugs across cell membranes $[44,45] .5 \sim 7 \%(>2,000)$ of all human genes 
code for transporters or transporter-related proteins [46]. Among all the transporters, ATP-binding cassette (ABC) and Solute carrier (SLC) transporters are two major families of membrane proteins that are important for transporting drugs. So far, more than 400 membrane transporters in these two families have been annotated in the human genome [47].

$\mathrm{ABC}$ transporters are a family of active transporters relying on adenosine triphosphate (ATP) hydrolysis to pump substrates in (influx) and out (efflux) of the cell membranes. In prokaryotes, $\mathrm{ABC}$ transporters function as both uptake transporters and efflux transporters. However, in eukaryotes, $\mathrm{ABC}$ transporters function only as efflux transporters $[48,49]$. The ABC gene family is composed of 49 genes in 8 subfamilies in the human genome [45]. The basic structure of ABC transporters contains two types of domains: nucleotide binding domain (NBD) and transmembrane domain (TMD) (Figure 1-4) [50]. The NBD, the conserved domain among the ABC transporters, plays a critical role in ATP binding and hydrolysis. Unlike NBD, TMD varies significantly in terms of the sequence, length, and number of transmembrane helices [51]. TMD binds to substrates and determines the transporter specificity through substrate-binding sites [52]. Among the ABC family of transporters, P-glycoprotein (P-gp/MDR $1 / A B C B 1)$, multidrug resistance protein $1(\mathrm{MRP} 1 / A B C C 1)$ and breast cancer resistance protein $(\mathrm{BCRP} / \mathrm{MXR} / A B C G 2)$ are three major members associated with multidrug resistance $[53]$.

P-gp, the product of $M D R 1$ (or $A B C B 1$ ) gene, was one of the first members of the $\mathrm{ABC}$ superfamily studied. Long before P-gp was discovered, it had been reported that incubating cancer cells with chemotherapy agents will generate subline cells that are resistant to not only the selecting agents but also to other structurally different agents [5355]. 10 years after the first report of the $170 \mathrm{kDa}$ glycoprotein [53-55], Roninson et al. reported the cloning of the gene encoding P-gp [56]. P-gp contains 2 NBDs and 12 TMDs (Figure 1-4) [50]. In addition to tumor cells, P-gp is also expressed in multiple normal organs/cells, such as intestinal enterocytes, kidney proximal tubule, hepatocytes (canalicular), and brain capillary endothelial cells. P-gp transports a broad variety of substrates out of the cells, including endogenous substrates, and drugs such as vincristine, vinblastine, doxorubicin, topotecan, mitoxantrone, etoposide, paclitaxel, docetaxel, and digoxin.

BCRP, a 72-kDa protein product of $A B C G 2$ gene, is also called ABCP or MXR. As indicated by its most commonly used name, BCRP was identified from a multidrug-resistant human breast cancer subline (MCF-7/AdrVp) in 1998. In 1999, it was cloned from mitoxantrone selected cells; thus it was also named mitoxantrone resistance protein (MXR) [57]. Interestingly, the clones from the drug selected cells containing single nucleotide mutations at the position of amino acid 482 ( $\mathrm{R}$ for wild-type protein, $\mathrm{T}$ in BCRP, and G in MXR), causing changes in substrate specificity [50, 58]. Unlike P-gp and MRPs, BCRP is a "half-transporter." It contains only 1 NBDs and 6 TMDs (Figure 1-4) [50]. BCRP is expressed in tumor cells, hematopoietic stem cells, placenta, small intestine, mammary glands, testis, liver, blood brain barrier, and the adrenal gland 


\section{Structure}

a

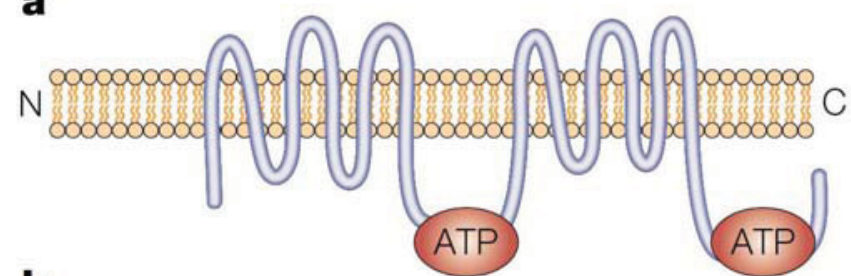

b

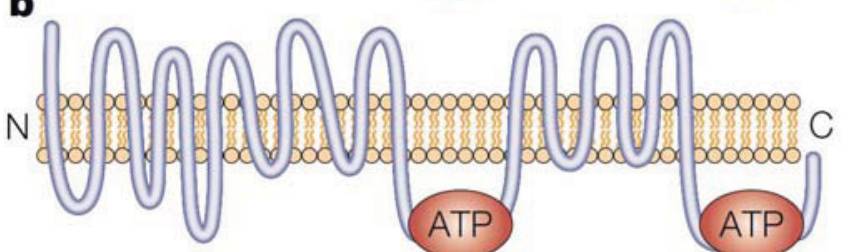

c

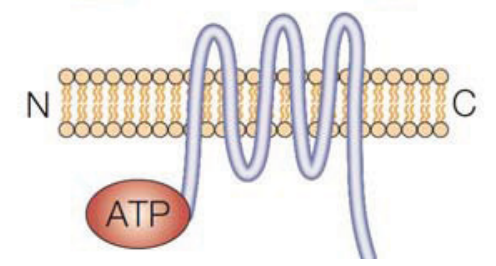

Examples

MDR1 (ABCB1)

MRP4 (ABCC4)

MRP5 (ABCC5)

MRP7 (ABCC1)

BSEP/SPGP (ABCB11)

MRP1 (ABCC1)

MRP2 (ABCC2)

MRP3 (ABCC3)

MRP6 (ABCC6)

MXR/BCRP/ABC-P

(ABCG2)

Figure 1-4. The structures of three categories of $\mathrm{ABC}$ transporter

a. $\mathrm{ABC}$ transporters such as multidrug resistance MDR1 and multidrug resistance associated protein 4 (MRP4) have 12 transmembrane domains and two ATP binding sites.

b. The structures of MRP1, 2, 3 and 6 are similar in that they possess two ATP binding regions. They also contain an additional domain that is composed of five transmembrane segments at the amino-terminal end, giving them a total of 17 transmembrane domains.

c. The 'half-transporter' ABCG2 contains 6 transmembrane domains and one ATP-binding region - in this case, on the amino-terminal side $(\mathrm{N})$ of the transmembrane domain. In other 'half-transporters', such as the transporter associated with antigen processing, the ATP-binding cassette is found on the carboxy-terminal (C) side of the transmembrane domain. Half-transporters are thought to homodimerize or heterodimerize to function.

Source: Reprinted with permission. Gottesman, M.M., T. Fojo, and S.E. Bates, Multidrug resistance in cancer: role of ATP-dependent transporters. Nature Reviews. Cancer, 2002. 2(1): p. 48-58. 
$[47,50,59]$. BCRP transports endogenous substrates and drugs such as mitoxantrone, topotecan, $\mathrm{SN}-38$, methotrexate, doxorubicin, and daunorubicin $[50,58]$.

In addition, P-gp, BCRP, and many other members of $\mathrm{ABC}$ transporter family play important roles in absorption, distribution, metabolism, and excretion (ADME) of chemotherapy drugs that are substrates of the $\mathrm{ABC}$ transporters.

\subsubsection{Role of $\mathrm{ABC}$ transporter in drug $\mathrm{ADME}$}

At least $10 \mathrm{ABC}$ transporters (P-gp, MDR3, BSEP, MRP1, MRP2, MRP3, MRP4, MRP5, MRP6, and BCRP) are involved in drug disposition [47, 59]. They are located on the apical or basolateral side of endothelial or epithelial cells in various organs (Table 1-1) $[47,59]$.

1.2.2.1. Effects on drug absorption. $A B C$ transporters located on the apical membrane of enterocytes, such as P-gp, MRP2, and BCRP [47], can pump their substrates back to the intestinal lumen - thus limiting the absorption of some orally administered drugs. For example, paclitaxel bioavailability in P-gp knockout mice increased from $11.2 \%$ to $35.2 \%$ compared to the wild-type mice [60]. Docetaxel bioavailability increased from $3.6 \%$ to $22.7 \%$ in P-gp knockout mice compared to the wild-type mice [61]. Leggas et al. reported that topotecan bioavailability increased in both BCRP and P-gp knockout mice and further increased with gefitinib (an inhibitor of BCRP and P-gp) treatment [62]. MRP1 and MRP3 are expressed at the basolateral side. Thus, theoretically, they could increase absorption of some drugs. A recent study suggests that MRP1 might facilitate the absorption of cobalamin in mice [63]. Several $\mathrm{ABC}$ transporters are expressed in the lungs and therefore may potentially affect the absorption of inhaled drugs. However, in vivo evidence is needed to support this hypothesis [64].

1.2.2.2. Effects on drug distribution. $A B C$ transporters located on the blood-brain barrier (BBB), blood-cerebrospinal fluid (CSF) barrier, blood-placental barrier, blood-testis barrier [65], blood-retina barrier [66], and heart [67] can limit the drug distribution to corresponding organs. $\mathrm{BBB}$ is a good example on how $\mathrm{ABC}$ transporters affect drug distribution. Before the 1990s, the BBB was considered a physical barrier formed by tight junctions between brain capillary endothelial cells (BCEC) that lack fenestrations [68]. Now, it is well established that P-gp on the apical membrane of BCECs is also an important component of BBB. The brain accumulation of many P-gp substrates can be much higher in P-gp knock-out mice than in wild-type mice, and inhibiting P-gp can increase the brain distribution. For example, amprenavir, an HIV protease inhibitor, is a substrate of P-gp [69] but not a substrate of BCRP [70]. Brain concentrations of $\left[{ }^{14} \mathrm{C}\right]$-amprenavir were 27 -fold higher in $m d r l \mathrm{a}^{-/-} / 1 \mathrm{~b}^{-/-}$mice compared to the wild-type mice. In the presence of P-gp and BCRP inhibitor GF120918, a 13-fold increase of $\left[{ }^{14} \mathrm{C}\right]$-amprenavir brain concentrations compared to the vehicle control treated 


\section{Table 1-1. Important $\mathrm{ABC}$ transporters}

\begin{tabular}{|c|c|c|}
\hline Name & Polarity & Locations \\
\hline P-gp & Apical & $\begin{array}{l}\text { Kidney, adrenal gland, liver, pancreas, intestine, } \\
\text { lung, BBB, placenta, prostate, skin, heart, } \\
\text { skeletal muscle, ovary, testis, retina }\end{array}$ \\
\hline MDR3 & Apical & Liver \\
\hline BCRP & Apical & $\begin{array}{l}\text { Placenta, mammary gland, BBB, liver, intestine, } \\
\text { kidney, lung }\end{array}$ \\
\hline MRP1 & $\begin{array}{l}\text { Apical (placenta, BBB) } \\
\text { Basolateral (others) }\end{array}$ & $\begin{array}{l}\text { Kidney, lung, testis, skeletal muscle, heart, } \\
\text { placenta, liver, intestine, brain }\end{array}$ \\
\hline MRP2 & Apical & Liver, kidney, intestine, placenta \\
\hline MRP3 & Basolateral & $\begin{array}{l}\text { Adrenal gland, intestine, pancreas, intestine, } \\
\text { gallbladder, placenta, liver, kidney }\end{array}$ \\
\hline MRP4 & $\begin{array}{l}\text { Apical (kidney, BBB) } \\
\text { Basolateral (prostate, } \\
\text { choroid plexus) }\end{array}$ & Prostate, kidney, liver, brain, pancreas \\
\hline MRP5 & $\begin{array}{l}\text { Apical (BBB) Basolateral } \\
\text { (others) }\end{array}$ & Heart, brain, neurons \\
\hline MRP6 & Basolateral & $\begin{array}{l}\text { Liver, kidney, skin, lung, heart, intestine, } \\
\text { pancreas, stomach }\end{array}$ \\
\hline BSEP & Apical & Liver \\
\hline
\end{tabular}

Sources: Adapted with permission.

1. Giacomini, K.M., et al., Membrane transporters in drug development. Nature Reviews. Drug Discovery, 2010. 9(3): p. 215-36.

2. Marquez, B. and F. Van Bambeke, ABC multidrug transporters: target for modulation of drug pharmacokinetics and drug-drug interactions. Current Drug Targets, 2011. 12(5): p. 600-20. 
group was observed [71]. Blood concentrations of $\left[{ }^{14} \mathrm{C}\right]$-amprenavir increased 1.1-fold and 2-fold in $m d r l \mathrm{a}^{-/ /} / 1 \mathrm{~b}^{-/-}$mice and GF120918 treated mice, respectively [71]. Although less prominent than P-gp, BCRP and some MRPs are also involved in the function of $\mathrm{BBB}$. These transporters can protect the brain from peripheral toxins but also hinder the delivery of central nervous system (CNS) drugs.

1.2.2.3. Effects on drug metabolism. $A B C$ transporters do not metabolize drugs themselves, but they affect metabolic clearance remarkably through interplay with drug metabolism enzymes. It has been proposed that $\mathrm{ABC}$ transporters and drug metabolism enzymes have undergone co-evolution toward a united xenobiotic defense system [45]. The relationship between P-gp and CYP3A4 is considered evidence of this theory. The $A B C B 1$ gene (encodes for P-gp) and the cluster of CYP $3 A 4$ genes are both at chromosome 7 and just $119 \mathrm{~kb}$ apart [45]. In addition, both genes are regulated by pregnane X receptor (PXR). P-gp and CYP3A4 also share similar substrate specificity and are co-localized in important drug-eliminating organs such as liver, kidney, intestine, and lung $[45,72]$. The most well-known effect of P-gp on CYP3A4 is exemplified by the intestinal first-pass metabolism. As mentioned, P-gp in enterocytes can pump back its substrate into the gut lumen, thus decreasing the fraction of absorption (fa). In addition, the P-gp-mediated efflux also helps decrease the likelihood of CYP3A4 saturation by lowering the intracellular peak drug concentration. The effluxed drug may undergo re-absorption, but the overall effect will be more opportunities for the CYP3A4 to metabolize the substrate drug. Therefore, P-gp can indirectly decrease the fraction of the intact drug escaping gut metabolism (fg) $[59,68,73]$. Drug conjugates formed by Phase II drug metabolism enzymes, e.g., UDP-glucuronosyltransferases (UGTs), sulfotransferases (SULTs), and glutathione S-transferases (GSTs), can also be effluxed by MRP2 and BCRP. This process is sometimes called "Phase III drug metabolism".

1.2.2.4. Effects on drug excretion. Drug excretion refers to the final removal of intact drugs or their metabolites from the body. Although this step can take place in several organs, biliary excretion and renal excretion are the most important routes [59]. Different $\mathrm{ABC}$ transporters are localized at the canicular or sinusoidal membrane of hepatocytes and in the kidney. Sinusoidal membrane transporters MRP1, MRP3, MRP4, and MRP6 extrude some drug metabolites (in most cases) or intact drugs back to the blood, and present them to bile or renal excretion. On the other hand, canicular membrane transporters P-gp, MRP2, and BCRP excrete their substrates directly into bile. P-gp is mainly responsible for cationic drugs or metabolites, while MRP2 and BCRP are for anionic drugs or metabolites [59]. However, there are also some controversies on the importance of MRP2 and BCRP, since it is difficult to differentiate their contribution from OATPs. ABC transporters are also located at both the apical and basolateral membrane of the renal epithelial cells. P-gp, MRP2, and MRP4 are expressed at the proximal tubular basolateral membrane facilitating the excretion of compounds into the urine. It seems that P-gp is responsible for excretion of digoxin and some hydrophobic cationic drugs, while MRP2 and MRP4 are for anionic drugs or metabolites. MRP1 is expressed at the apical cell membrane of distal tubules and collecting ducts. It may be 
part of a mechanism to prevent drug accumulation (which is toxic to nephron) after water re-absorption in that area.

\subsubsection{Role of $\mathrm{ABC}$ transporter on drug resistance}

Resistance to chemotherapy is one of the major hindrances to the current multimodal cancer treatment paradigms. The resistance can be either a result of changes in drug's ADME at a non-cellular level or the consequence of certain mechanisms within tumor cancer cells [74]. As implied by the names of their well-known members (e.g., Breast Cancer Resistance Protein, Multidrug Resistance Proteins, as well as the gene name of P-gp, Multidrug Resistance gene 1), ABC transporters are involved in drug resistance in cancers.

As mentioned in the previous section, some ABC transporters affect ADME of chemotherapeutic agents. Therefore, induction of these transporters by the chemotherapeutic agent itself or other co-administrated drugs can cause resistance to that agent at the non-cellular level. This can work together with other drug resistance mechanisms at this level, e.g., drug metabolism enzymes induction in drug-handling organs as well as poor vascularization and acidic $\mathrm{pH}$ in solid tumors $[75,76]$.

Cellular drug resistance can be further stratified into two types: the resistance to a class of drugs with a similar mechanism of action, and the resistance to various drugs with different structures and targets [75]. The former type of resistance obviously results from changes in drug targets, while the latter type, so-called multiple drug resistance (MDR), has been a topic of much discussion.

Multiple mechanisms have been proposed for MDR, including reduced sensitivity to apoptosis and existence of cancer stem cells, as well as high levels of expressions of drug metabolism enzymes (such as GST) and ABC transporters in cancer cells [74, 76]. MDR caused by ABC transporters is called "transport MDR" or "classical MDR" while MDR by other mechanisms is referred as "atypical MDR" or "non-classical MDR" [74, 76].

All 10 aforementioned ABC transporters that are important for ADME plus 3 additional members (MRP7, MRP8, and ABCA2) have been shown to cause drug resistance in cell lines in vitro $[77,78]$. In many different cancer tissues from both drug-naïve and treated patients, various ABC transporters frequently can be detected [79]. However, it is more challenging to get a clear picture of their significance in clinical MDR. In order to discern the contribution of $\mathrm{ABC}$ transporters from other factors, correlation analysis between poor chemotherapy outcome and expression of $\mathrm{ABC}$ transporters in a large cohort of cancer patients is a necessity. Another reason that makes the consensus on clinical relevance of some transporters hard to achieve is the large variety of tumor types and drug treatments [79]. 
Currently, most studies on the relationship between $\mathrm{ABC}$ transporters and clinical MDR are focused on P-gp, MRP1, and BCRP, which are also the most extensively studied ones in drug ADME. Correlation between P-gp expression and prognosis of chemotherapy in breast cancer, sarcoma, and acute myelogenous leukemia (AML) are well established [80]. However, the results of clinical trials using specific P-gp inhibitors are not very promising [81].

The expression level of MRP1 does not correlate with the clinical MDR of AML. Its significance on the chemotherapy outcome of chronic lymphocytic and promyelocytic leukemia, non-small cell lung cancer (NSCLC), and breast cancer has been a point of controversy [80]. There are conflicting data regarding the role of BCRP in clinical MDR in leukemia [53, 80, 81]. No or little clinical relevance of BCRP expression was found in breast cancer, ovarian cancer, and locally advanced bladder cancer. Correlation between BCRP expression and adverse prognosis of lung cancer, esophageal cancers, and some lymphomas has been reported [82].

Recently, the cancer stem cells paradigm has been incorporated into the drug resistance concept. Cancer stem cells are drug-resistant pluripotent cells expressing high levels of ABC transporters, especially BCRP. This subpopulation can survive chemotherapy when the other committed non-resistant cancer cells in the original tumor mass are killed and therefore serve as an unrestricted reservoir for drug resistant tumor relapse $[80,83]$. Thus, some conclusions on the relevance of BCRP in clinical MDR need to be re-evaluated, since BCRP expression in cancer stem cells was not examined in many previous solid tumor studies [82]. Some researchers have proposed that the failure in P-gp inhibitor development may be due to missing an important target - the BCRP [84].

\subsubsection{Mechanism of action of $\mathrm{ABC}$ transporters}

Current understanding of the mechanism of action of $\mathrm{ABC}$ transporters is briefly summarized below (Figure 1-5) [50, 51, 85-90]. As mentioned before, TMDs of ABC transporters bind to drug substrates, while NBDs bind to ATP. Binding of substrate to the TMD stimulates the ATPase activity. ATPase facilitates ATP hydrolysis and releasing of inorganic phosphate (Pi) and adenosine diphosphate (ADP). ATP hydrolysis provides the energy to cause a conformational change at the TMDs. TMDs will change from inward-facing conformation (facing the inside) to outward-facing conformation (facing the outside) and release substrate to the extracellular space. Experimental data supported that two ATP hydrolysis events are needed to transport one drug molecule. These two events do not happen simultaneously. The first ATP hydrolysis is needed to transport the substrate and the second ATP hydrolysis is needed to "reset" the transporter from outward facing back to forward facing so that the transporter can bind substrate again [91]. Formation of a homodimer or heterodimer is a prerequisite for functionality of half-transporters such as BCRP [50, 58, 92].

Based on this mechanism of action, the ATPase assay was developed to indirectly 


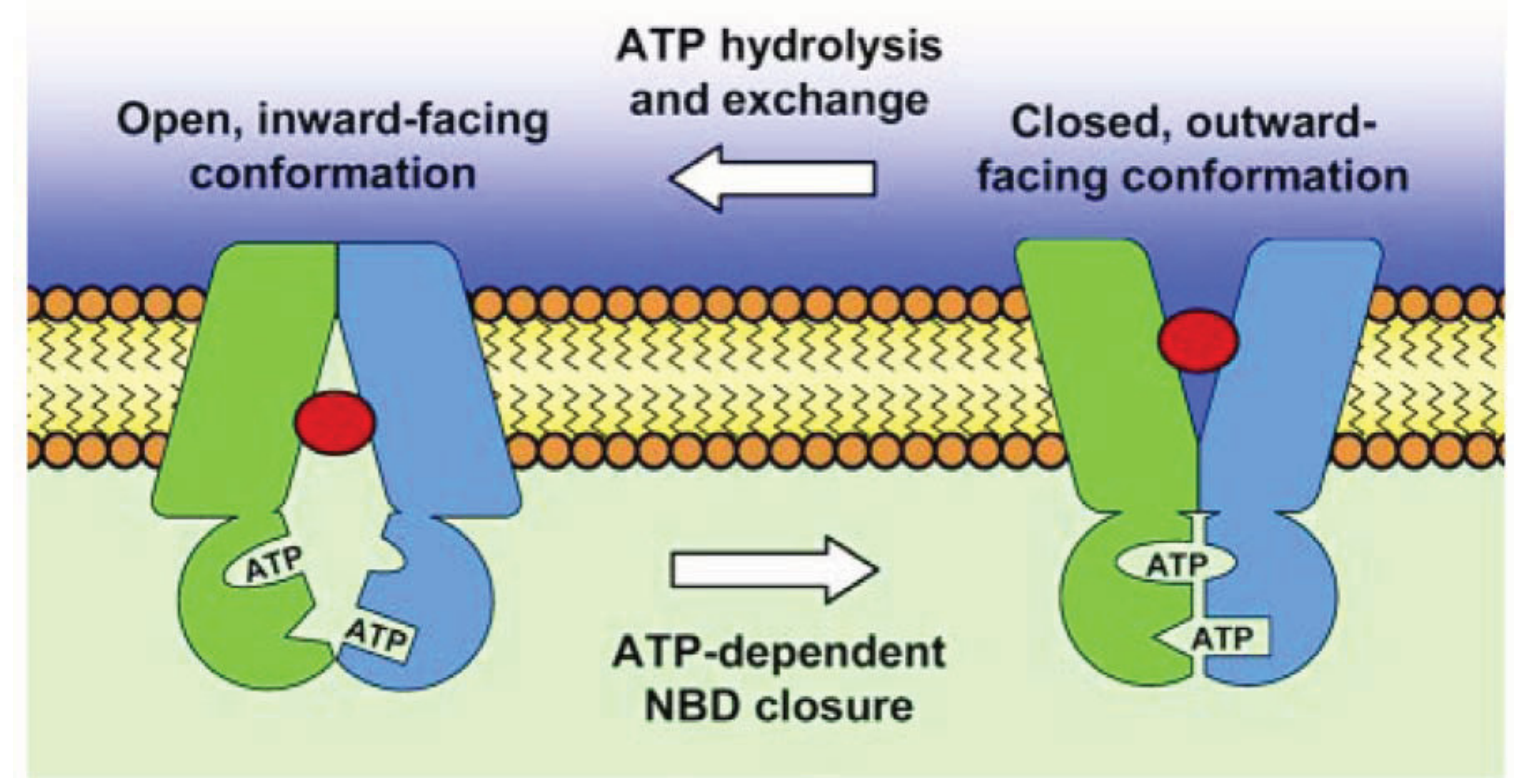

Figure 1-5. Mechanism of action of a typical ABC transporter

ATP dependent closure/dimerization of cytosolic NBDs provides the power stroke that pulls the TMDs from an inward- to outward facing conformation. Both exporters and importers probably use the same basic mechanism but shift which state binds the transport substrate (red) with high affinity. Most eukaryotic ABC transporters are heterodimers, with 2 homologous but nonequivalent halves (green and blue).

Source: Reprinted with permission. Procko, E., et al., The mechanism of $A B C$ transporters: general lessons from structural and functional studies of an antigenic peptide transporter. The FASEB Journal, 2009. 23(5): p. 1287-302. 
detect the activity of $\mathrm{ABC}$ transporters to determine substrates and inhibitors of $\mathrm{ABC}$ transporters. The ATPase assay contains two modes, activation mode and inhibition mode. Under activation mode, ABC transporter substrates bind to the TMD and stimulate ATPase activity, which further hydrolyzes ATP and releases Pi, which can be detected by a colorimetric reaction and is proportional to the ATPase activity. Increased Pi release suggests that a compound is a substrate of $\mathrm{ABC}$ transporters. Under inhibition mode, $\mathrm{Pi}$ release is detected in the presence of a known $\mathrm{ABC}$ transporter substrate with or without a test compound. If the test compound is an inhibitor of the transporter or slowly transported substrate, Pi release stimulated by the transporter substrate will be reduced $[93,94]$.

\subsubsection{Effect of p53 on $\mathrm{ABC}$ transporters}

Conflicting data suggested that p53 may also regulate the expression of $\mathrm{ABC}$ transporters $[95,96]$. In 1992, Chin et al. first reported that the MDR1 promoter was repressed by wild-type p53 [97]. Later, Stauss et al. reported that the MDR1 downstream promoter contains a wild-type p53-binding site [98]. Unlike wild-type p53, mutant p53 was found to activate the $M D R 1$ promoter [97]. Similarly, Zastawny et al. also reported that wild-type p53 represses $M D R 1$ promoter activity, and mutant p53 enhances $M D R 1$ promoter activity [99]. Further study showed the overlapping region on the MDRI promoter for mutant p53 transactivation and for basal promoter activity [100]. A study by Sampath et al. suggested while mutant p53 requires an Ets-1 transcriptional factor binding site to regulate $M D R 1$ promoter transcriptionally, wild-type p53 does not interact with Ets-1 [101]. The increased $M D R 1$ expression could be caused by both a loss of p53 repression on $M D R 1$ promoter and an increased transactivation of $M D R 1$ through a "gain of function" of mutant p53 [102]. However, the role of p53 in regulating MDR 1 is still controversial $[95,102]$. Transfection of wild-type p53 expression had been reported to stimulate $M D R 1$ promoter in p53 negative cell lines [103] or show no (or marginal) change in $M D R 1$ gene expression and function [104, 105].

In addition to $M D R 1$, wild-type p53 has been reported to suppress MRP1 reporter activity [106]. Unlike MDR1, mutant p53 did not up-regulate MRP1 [101, 106]. Data about regulation of MRP1 by p53 is also conflicting. Wild-type p53 enhanced MRP expression and activity instead of suppressing MRP1 expression in three of the five cell

lines, and no change was observed in other two of the five cell lines [96]. Recently, Wang et al. reported that wild-type, but not mutant $\mathrm{p} 53$, can reduce the expression of BCRP in MCF-7 cells [107].

Regulation of ABC transporter expression by p53 can have a functional effect on drug resistance. For example, wild-type p53 inactivation (introduced by expressing dominant negative mutant $\mathrm{p} 53$ ) increased the expression of MDR1. This leads to decreased uptake and increased resistance to vinblastine [102]. Increased vincristine sensitivity after changing the cells from expressing dominate negative mutant p53 to wild-type p53 was also reported [96]. 


\subsubsection{Evaluation of transporter mediated drug-drug interaction}

Because of the important roles of transporters on drug disposition, inhibition or activation of $\mathrm{ABC}$ transporter(s) may alter the PK of a compound that is substrate(s) of $\mathrm{ABC}$ transporter(s). For example, oral co-administration of P-gp inhibitor clarithromycin can lead to a $64 \%$ increase of AUC of P-gp substrate digoxin, which explains the clinical cases of clarithromycin-induced digoxin toxicity [108]. P-gp inhibitor dronedarone resulted in a 157\% increase of digoxin AUC [47]. BCRP and P-gp inhibitor GF120918 resulted in a 143\% increase of oral topotecan AUC [47]. Therefore, in vitro and sometimes in vivo follow-up studies are needed to understand transporter-mediated drug-drug interaction for avoiding serious adverse events.

In 2006, the Food and Drug Administration (FDA) published guidance for studying transporter-based drug-drug interactions. A decision tree regarding P-gp-mediated drug-drug interaction was included in the guidance [47]. In 2010, the International Transporter Consortium further broadened the decision tree (Figure 1-6) [47]. Approaches to answer important questions were addressed for both ABC transporters (P-gp and BCRP) and SLC transporters (organic cation transporter (OCT) and the organic anion transporter (OAT)) [47]. These questions include, for example, which transporters are important for drug absorption and disposition, what in vitro methods are recommended to evaluate drug-transporter interaction, and what are the criteria for conducting clinical studies based on in vitro data.

For P-gp and BCRP, the bidirectional transport assay using polarized monolayer cells is one of the important preferred functional studies to identify if a compound is a substrate and/or inhibitor of P-gp. Caco-2 cells are derived from human epithelial colorectal adenocarcinoma cells. When cultured under specific conditions, Caco-2 cells become differentiated, polarized, and functionally resemble the morphology, polarity, and expression patterns of the transporters and enzymes of the small intestine [109]. Caco-2 cells have been widely used as an in vitro model to evaluate intestinal drug absorption and efflux. Transporter transfected Madin-Darby canine kidney epithelial (MDCK) cells or porcine kidney epithelial cells (LLCPK1) cells are also routinely used in bidirectional transport assay. Similar to Caco-2 cells, these cells can also polarize and form a monolayer. Because P-gp and BCRP are expressed at the apical side of the polarized cells [109], B to A transport represents the passive diffusion and the efflux of a compound, and $\mathrm{A}$ to $\mathrm{B}$ transport represents the passive diffusion and the uptake of a compound. Therefore, it is important to look at both A to B and B to A direction for evaluating P-gp and BCRP efflux in the system. When interpreting data from these systems, one must consider that multiple endogenous drug transporters are expressed in Caco-2 (P-gp, BCRP, MRP1-6, ABCA1, ABCG1, HPT1 and many SLC family uptake transporters) [110], MDCK II (P-gp, MRP1, MRP2, and MRP5) [111, 112], and LLC-PK 1 (P-gp, MRP1 and MRP2) [113].

A decision tree for P-gp or BCRP substrate interactions is listed in Figure 1-6 [47]. An efflux ratio less than 2 indicates poor or non-P-gp (or non-BCRP) substrate. For 
Figure 1-6. Decision trees for studying transporter-based drug-drug interactions

A. Decision tree for P-gp or BCRP substrate interaction. A new molecular entity (NME) is considered to be a potential P-gp or BCRP substrate if the efflux ratio - basal to apical (B-A) to apical to basal (A-B) - is $\geq 2$ in an epithelia cell system that expresses one or both transporters (see (a) in the figure). A net flux ratio cut-off higher than 2 or a relative ratio to positive controls may be used to avoid false positives if a ratio of 2 is deemed non-discriminative as supported by prior experience with the cell system used. Additional corroboration that an NME may be a P-gp or BCRP substrate can be achieved with the use of inhibitors. Reduction of the flux ratio by the P-gp (or BCRP) inhibitors should be greater than $50 \%$ (see (b) in the figure). If the flux ratio is not reduced by P-gp (or BCRP) inhibitors, then other efflux transporters may be responsible for the observed net flux (see (d) in the figure).

B. Decision tree for transporter inhibitor interaction. [I]1 is the steady-state total Cmax at the highest clinical dose, and [I]2 is the theoretical maximal gastrointestinal drug concentration after oral formulation at the highest clinical dose in a volume of $250 \mathrm{ml}$.

Source: Adapted with permission. Giacomini, K.M., et al., Membrane transporters in drug development. Nature Reviews. Drug Discovery, 2010. 9(3): p. 215-36. 
A.

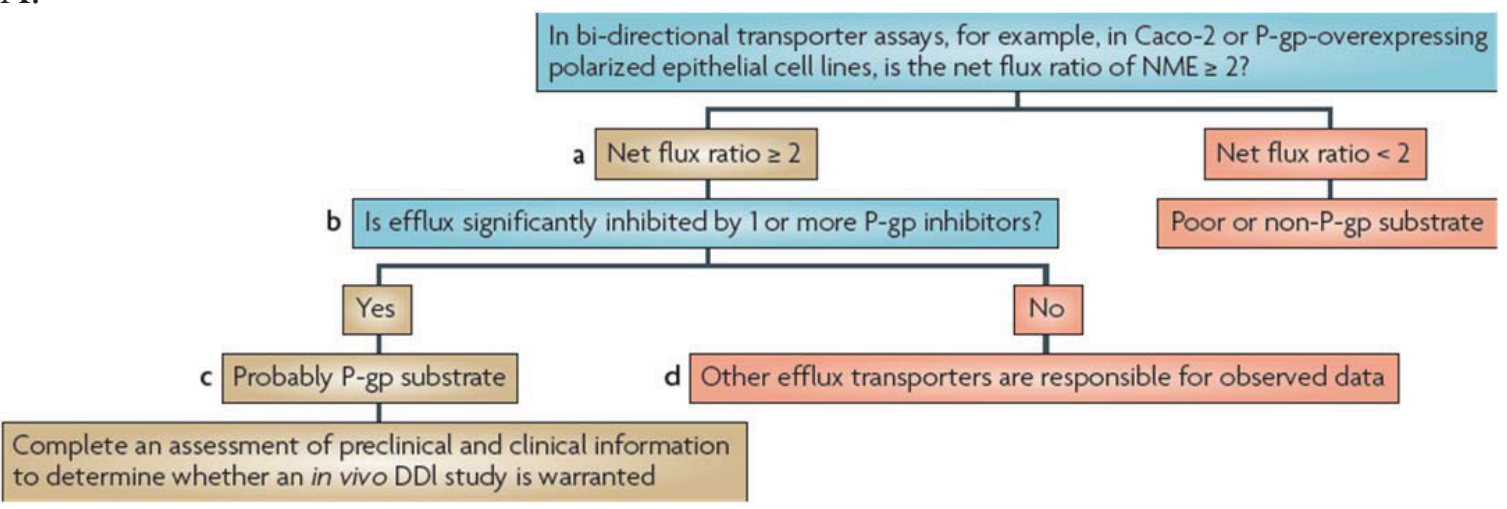

B.

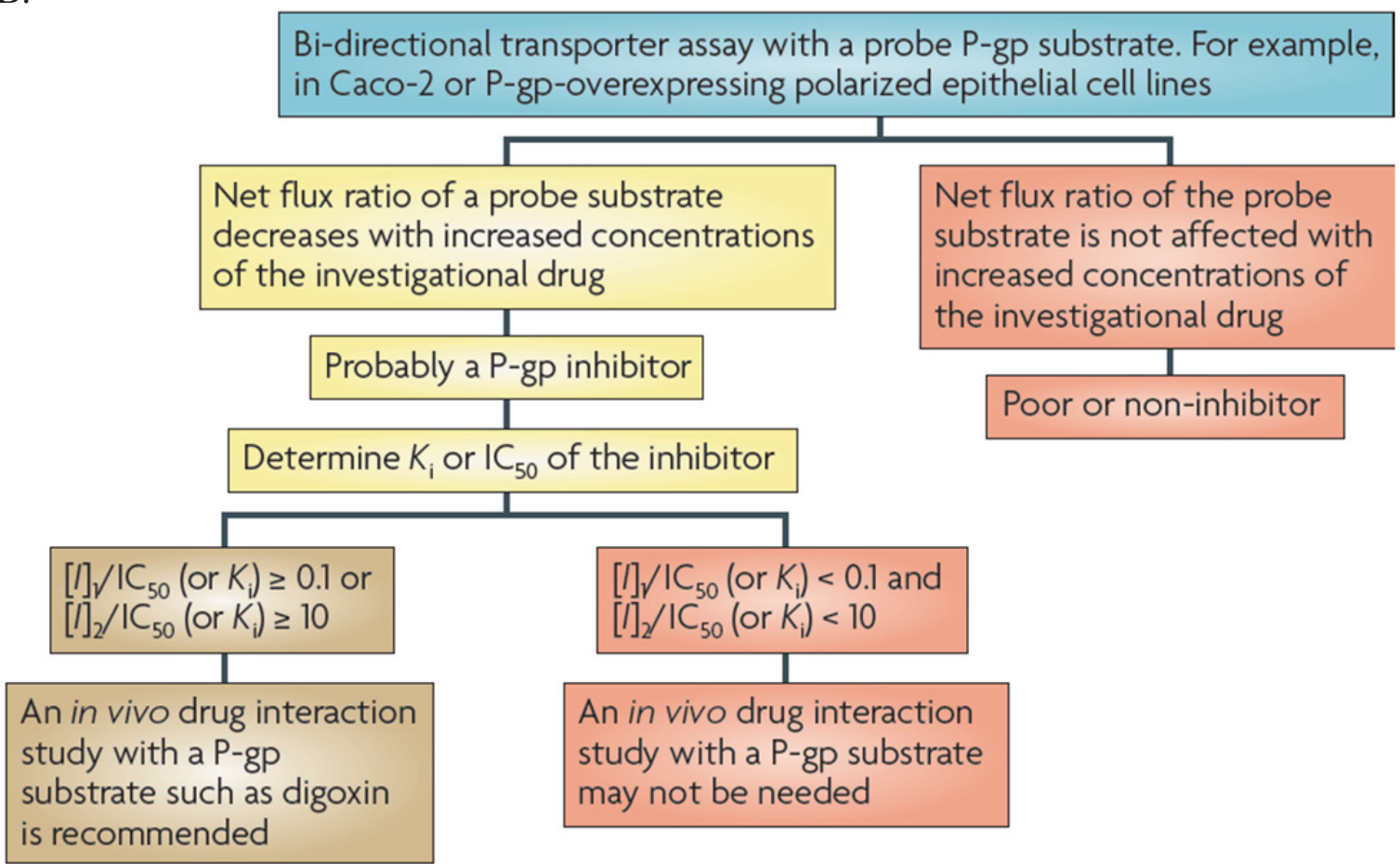


compounds with efflux ratio $\geq 2$, inhibitor (s) will be used. If the efflux ratio reduction is larger than $50 \%$ or the efflux ratio close to 1 can be achieved after adding P-gp (or BCRP) inhibitor(s) at effective concentration, the compound is probably a substrate of P-gp (or BCRP). Otherwise, other efflux transporters may be responsible for the high efflux ratio. If an in vitro study suggested that a compound is a substrate of $\mathrm{P}$-gp or $\mathrm{BCRP}$, an in vivo drug-drug interaction study may be needed, depending on the property of a compound. For a drug that falls into Biopharmaceutics Classification System (BCS) Class I (high solubility, high permeability) or Biopharmaceutics Drug Disposition Classification System (BDDCS) Class I (high solubility, extensive metabolism), even if it could be P-gp substrate in vitro (for example, verapamil), its bioavailability is not likely to be affected by P-gp modulators [114]. If disposition in kidney, brain, and tumor is not important for that drug, then no in vivo studies on drug-drug interaction are necessary [114].

If a compound reduced the efflux ratio of P-gp (or BCRP) in bi-directional transport assay, this compound might be a potential P-gp (or BCRP) inhibitor in vivo. The International Transporter Consortium recommended $[\mathrm{I}]_{1} / \mathrm{IC}_{50} \geq 0.1$ or $[\mathrm{I}]_{2} / \mathrm{IC}_{50}>10$ as cut-off values for further in vivo drug interaction studies (Figure 1-6B) [47]. $\mathrm{IC}_{50}$ is the in vitro $\mathrm{IC}_{50},[\mathrm{I}]_{1}$ is the steady-state unbound Cmax at the highest clinical dose, and $[\mathrm{I}]_{2}$ is the theoretical maximal gastrointestinal drug concentration after oral formulation at the highest clinical dose in a volume of $250 \mathrm{ml}$. If $[\mathrm{I}]_{1} / \mathrm{IC}_{50} \geq 0.1$ or $[\mathrm{I}]_{2} / \mathrm{IC}_{50}>10$ occur, an in vivo drug interaction study with P-gp or BCRP substrate is recommended. One possible substrate for an in vivo P-gp-mediated drug interaction study is digoxin, and possible substrates for an in vivo BCRP-mediated drug interaction study are sulphasalazine, rosuvastatin, pitavastatin, ciproflozacin, and dipyridamole.

\subsection{Physiologically-based Pharmacokinetic (PBPK) Modeling and Simulation in Drug Development}

\subsubsection{Introduction of PBPK model}

1.3.1.1. Classical and PBPK model. Disposition of a drug by the body is a complex process. A compound can be administered by various routes such as oral, intravenous, subcutaneous, intramuscular, or subconjunctival injection. Through various mechanisms of absorption, the drug is absorbed and then distributed to the tissues by the body fluids (e.g., blood, lymphatic system, CSF). The drug can be metabolized in the liver and/or other tissues to other compound(s) and/or eliminated unchanged through bile or the kidney. Protein binding in the plasma and different tissues can be different due to various protein contents. As discussed earlier, efflux and uptake transporters expressed throughout the body can impact the ADME process. In the same tissue, drug concentrations in the vascular, extracellular, and intracellular compartments can be different. Due to the complexity of this process, drug concentrations in the blood and in 
different parts of the body can be quite variable. Thus drug concentrations in the plasma do not always reflect target tissue concentrations.

An appropriate pharmacokinetic model that adequately describes and reasonably predicts the time-dependent drug concentrations in the body is critical in both preclinical and clinical drug development. Currently, two broad approaches to pharmacokinetic analysis are classical (sometimes called empirical or conventional) and PBPK models, although the two approaches do share similar features.

The classical pharmacokinetic model focuses mainly on the drug concentrations in blood (whole blood, plasma, or serum) or other easily accessible body fluids (urine, feces, CSF, or breast milk). It generalizes the complex drug distribution process into multiple theoretical compartments. An example of classic PK model structure is provided in Figure 1-7. Compartments in classical PK models do not represent real organ compartments and usually lack mechanistic insight. This limits their application in certain areas of drug discovery and development such as predicting human systemic and target tissue drug concentrations based on in vitro data or animal PK data, and predicting drug-drug interactions.

PBPK models, on the other hand, are more complex models that map the drug PK process onto physiologically realistic compartment structures. A typical structure of a PBPK model is given in Figure 1-8 [115]. In such a model, the body is usually modeled as a closed circulatory system consisting of tissues that are important for drug absorption, distribution, metabolism, elimination, or any other tissues that are of interest. Compartments that represent real tissues are connected by blood flow. In general, tissue-specific arterial blood flow serves as model input into the tissues and comes out of the tissues as venous blood, thus serving as model output, with some exceptions. For splanchnic organs such as stomach, intestine, spleen and pancreas, venous blood flows into the liver through the portal system. For the lung, the pooled venous blood flows in the lung and arterial blood flows out of the lung. In contrast to the classic PK model, the physiologically-based nature of the PBPK modeling allows us to address mechanistic questions with regard to the PK as well as extrapolating knowledge obtained from one species to another, including human.

However, the distinction between the classic and PBPK model is not always clear. As Nestorov mentioned in his reviews, it is impossible and unnecessary to define formally what a PBPK model is or to specify a clear distinction between the classic and PBPK model [116, 117]. The classic model (especially mechanistic PK model) contains more and more physiological information (e.g., including body weight in the allometric scaling, using bile empty time for modeling enterohepatic circulation). And the PBPK model usually incorporates classic PK model components. For example, PBPK modeling software PK-sim ${ }^{\circledR}$ incorporates the animal clearance value from classic PK model into the PBPK model to predict concentration time profile both in animal and in human. Also, one important step for establishing the whole body PBPK model is to generate PK model in the plasma using classic PK modeling. To distinguish between a predominantly classical and predominantly PBPK model, the convention is to look at the model 


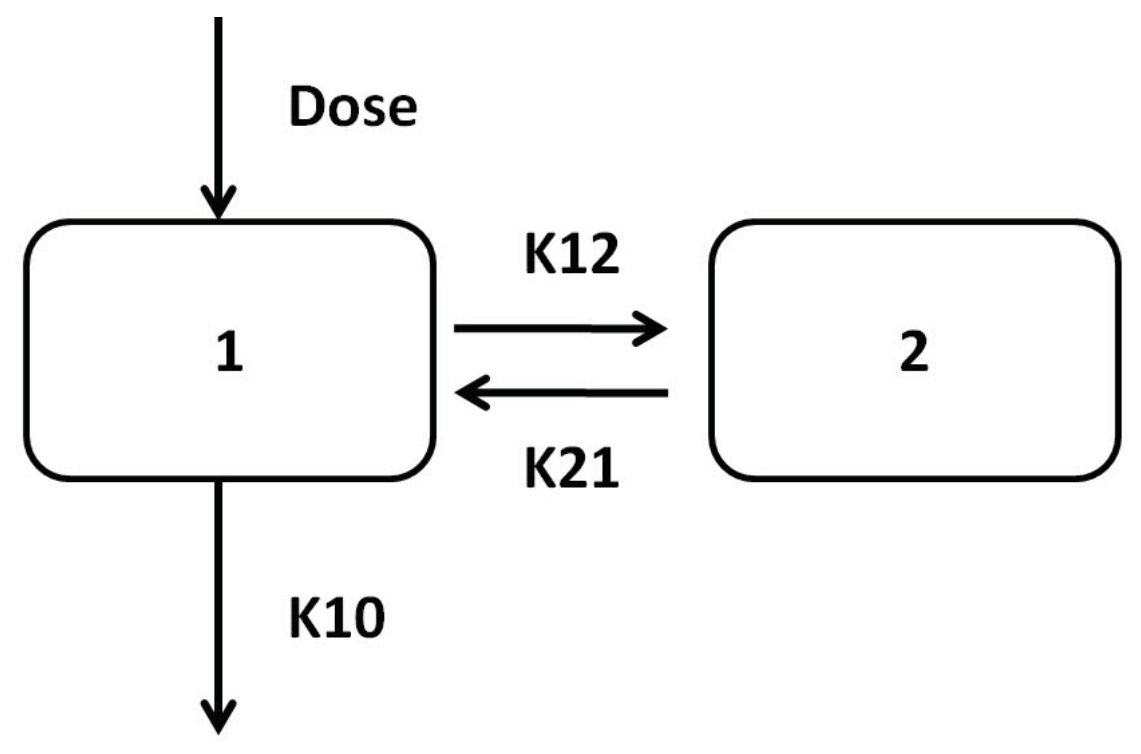

Figure 1-7. Example model structure of the classical PK model 


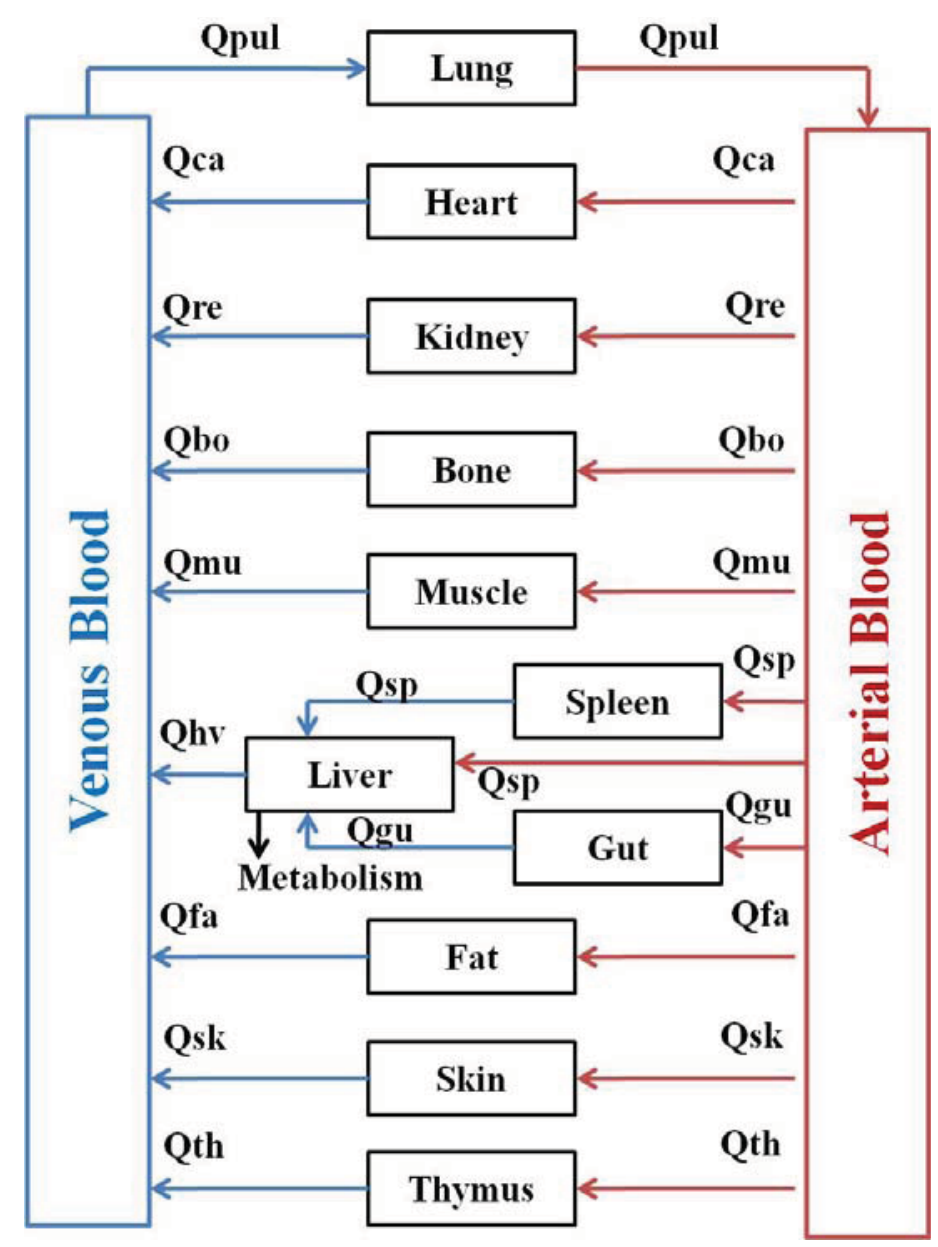

Figure 1-8. Typical structure of a whole-body PBPK model

Q refers to blood flow: to the lungs (Qpul), the heart (Qca), the kidneys (Qre), the bones (Qbo), the muscles (Qmu), the spleen (Qsp), the liver (Qha), the hepatic vein (Qhv), the gut (Qgu), the fat (Qfa), the skin (Qsk), and the thymus (Qth).

Source: Adapted with permission. Kawai, R., et al., Physiologically based pharmacokinetics of cyclosporine A: extension to tissue distribution kinetics in rats and scale-up to human. The Journal of Pharmacology and Experimental Therapeutics, 1998. 287(2): p. 457-68. 
structure. As mentioned in a review by Nestorov, if the model structure precedes the analysis of the compound specific data, and predominantly represents actual tissue or organ spaces, then it can be classified as a PBPK model [117]. It should be mentioned that both the classical PK model and the PBPK model belong to the compartment PK model hierarchy because both models classify the body into a number of subunits called "compartments" [117, 118].

1.3.1.2. PBPK application area. Due to the advances in computing power, PBPK models have been increasingly used in recent years [119]. The majority of all PBPK related publications $(60 \%)$ deal with issues pertaining to risk assessment of environmental chemicals [120]. Because the PBPK model is more mechanistically, anatomically, and physiologically relevant than the classic compartment modeling, the established PBPK model from animal study can be used to extrapolate to humans.

In the drug discovery and development area, there is a particular interest to use the PBPK model for estimating the human PK of drug candidates from in silico, in vitro, or in vivo animal PK data and to select the most promising compounds for further development. Methods for predicting human PK are quite variable, and many methods are under evaluation. For example, detailed scaling methods can be found in recently published papers from the Pharmaceutical Research and Manufacturers of America (PhRMA) group, including 24, 29, and 66 methods for predicting human volume of distribution, human clearance and oral area under the curve, respectively [121-125], based on in vitro and animal PK data. PBPK model has also been used for special population — such as pediatrics [126-134], elderly patients [135, 136], pregnant [137-139] and lactating [140,141] individuals, and for patients with organ impairments [142]. It has been applied to predict human drug-drug interaction [130, 143-151], inter-individual variability [120], and the effect of genetics [152-154]. In addition to small molecules, the applications of the PBPK model have also been extended to large molecules [155-163] and nano-particles [164-167].

1.3.1.3. PBPK model software. Software is a critical tool for establishing PBPK models and performing simulations. There are a number of commercial software products developed for building PBPK models, including Simcyp (Simcyp Ltd., Sheffield, UK), GastroPlus $^{\mathrm{TM}}$ (Simulations Plus Inc, Lancaster, CA) and PK-Sim ${ }^{\circledR}$ (Bayer Technology Services, Leverkusen Germany). These products usually do not require code writing and are capable of performing complex IVIVE simulation, DDI prediction, and extrapolating PK parameters from adults to pediatric population without in vivo experiment data. Software such as SAMM II, NONMEM, ADAPT, and Phoenix (WinNonlin 6) can be used for both conventional PK and PBPK modeling. Different software requires different coding. In some cases, such as in SAAMII and Phoenix WinNonlin 6, writing code may not be needed. Software products such as MATLAB have powerful programming flexibilities. 


\subsubsection{Basic concepts of PBPK modeling}

1.3.2.1. Major components of PBPK model. Inputs to PBPK models comprise drug-independent and drug-dependent information. Organ mass or volume, blood flow, tissue composition, and the anatomical arrangement of the tissues and organs of the body are drug-independent components. Drug-dependent information includes partition coefficients, protein binding, PK properties, membrane permeability, enzymatic stability, and transporter-drug relationships. Since drug-independent components and many of the drug-dependent components are not required in the classical PK model, it is believed that the PBPK model contains richer informational content.

Besides data collection, the general procedure for developing PBPK models consists of three major steps [116]: 1) specification of whole body structure and the tissue structure, 2) writing differential equations, and 3) estimating parameters.

\subsubsection{Specification of whole body structure and tissue structure}

1.3.2.2.1. Specification of whole body structure. To establish a PBPK model for a specific compound, the whole body structure needs to be built to meet specific study purposes. It is important to decide which tissues/organs to include in the model. On one hand, the PBPK model should contain a large amount/number of tissues/organ that are important for drug ADME and tissues of special interest. On the other hand, for practical reasons, too many tissue/organ compartments not only increase the need for a lot of experimental data and literature information but also increase the difficulty of the mathematical calculations. Although currently there is no definite rule for selection of the tissues to be included, in general "core tissues" such as blood, liver, kidney, adipose, and tissues of interest are included in the model [116]. All the rest of the tissues can be lumped into "rapid equilibrating" or "slow equilibrating" compartments. When both a parental drug and its metabolite(s) are studied, separate PBPK models should be developed for both the drug and the metabolite(s). The models are then linked through the metabolism compartment (usually liver) with one part of the parent drug elimination output serving as input for the metabolite(s) [168, 169].

1.3.2.2.2. Specification of tissue structure. After a whole body structure is defined, the next step is to specify the structure of a specific tissue. In most cases, a simple perfusion limited model is used for the tissues (Figure 1-9A) [168]. The underlying assumption is that the drug distributes instantly in the tissues and there is no concentration gradient existing in the tissues. Although making this assumption oversimplifies the real situation, it has the major advantage of reducing the model complexity. When permeability limits the distribution of a drug within a tissue, a diffusion-limited model can be applied (Figure 1-9B) [168]. One tissue compartment can be divided further into two or three compartments. For a two compartment model in a 
Figure 1-9. $\quad$ Structures and equations for individual organ models

A. Perfusion-limited model for non-eliminating organs. $\mathrm{V}_{\mathrm{T}}$ is the total volume of organ $\mathrm{T}$; $\mathrm{Q}_{\mathrm{T}}$ is the blood flow to the organ; $\mathrm{C}_{\mathrm{T}}$ is the concentration of the drug in the organ; $\mathrm{R}_{\mathrm{T}}$ is the partition coefficient; $\mathrm{C}_{\mathrm{Art}}$ is the concentration of drug in arterial blood; $\mathrm{C}_{\mathrm{T}}$, ven is the venous effluent drug concentration $\left(\mathrm{C}_{\mathrm{T} \text { Ven }}=\mathrm{C}_{\mathrm{T}} / \mathrm{R}_{\mathrm{T}}\right)$. In this model, the measured tissue concentration is $\mathrm{C}_{\mathrm{T}}$.

B. Diffusion-limited model for non-eliminating organs. $\mathrm{C}_{\mathrm{T}, \mathrm{V}}$ and $\mathrm{V}_{\mathrm{T}, \mathrm{V}}$ represent the drug concentration and volume for the organ vascular space; $\mathrm{C}_{\mathrm{T}, \mathrm{EV}}$ and $\mathrm{V}_{\mathrm{T}, \mathrm{EV}}$ represent the corresponding terms for the extra-vascular space; $\mathrm{k}_{\mathrm{V}, \mathrm{EV}}$ and $\mathrm{k}_{\mathrm{EV}, \mathrm{V}}$ represent the drug transport rates between the vascular and extra-vascular spaces; $f_{u b}$ is the fraction of unbound drug in the vascular space. In this model, the measured tissue concentration is $\mathrm{C}_{\mathrm{T}, \mathrm{EV}}$.

C. Perfusion-limited model for eliminating organ. $\mathrm{CL}_{\text {int }}$ represents the intrinsic clearance of the drug.

D. Diffusion-limited model for eliminating organs. $\mathrm{CL}_{\mathrm{int}} \mathrm{T}$ represents the intrinsic clearance of the drug.

Source: Adapted with permission. Xu, L., et al., Physiologically-based pharmacokinetics and molecular pharmacodynamics of 17-(allylamino)-17-demethoxygeldanamycin and its active metabolite in tumor-bearing mice. Journal of Pharmacokinetics and Pharmacodynamics, 2003. 30(3): p. 185-219. 
A Perfusion limited model for non-eliminating organs

$\stackrel{C_{T, \text { Ven }}}{\longleftarrow Q_{T}} \begin{gathered}C_{T} \\ R_{T} V_{T}\end{gathered} \quad V_{T} \frac{C_{A r t}}{d t}=Q_{T}\left(C_{A r t}-\frac{C_{T}}{R_{T}}\right)$

B Diffusion limited model for non-eliminating organs

\begin{tabular}{|c|c|c|c|}
\hline$C_{T, V e n}$ & $\overbrace{V, E V \_} C_{T, V} \quad V_{T, V}$ & $C_{A r t}$ & $V_{T V} \frac{d C_{T, V}}{d t}=Q_{T}\left(C_{A r r}-C_{T V}\right)-k_{V, E V} f u_{b} C_{T V} V_{T V}+k_{E V V} C_{T, E V} V_{T}$ \\
\hline$Q_{T}$ & ${ }_{k_{E V, V}} C_{T, E V} V_{T, E V}$ & $Q_{T}$ & $V_{T, E V} \frac{d C_{T, E V}}{d t}=k_{V, E V} f u_{b} C_{T, V} V_{T, V}-k_{E V, V} C_{T, E V} V_{T, E V}$ \\
\hline
\end{tabular}

C Perfusion limited model for eliminating organs

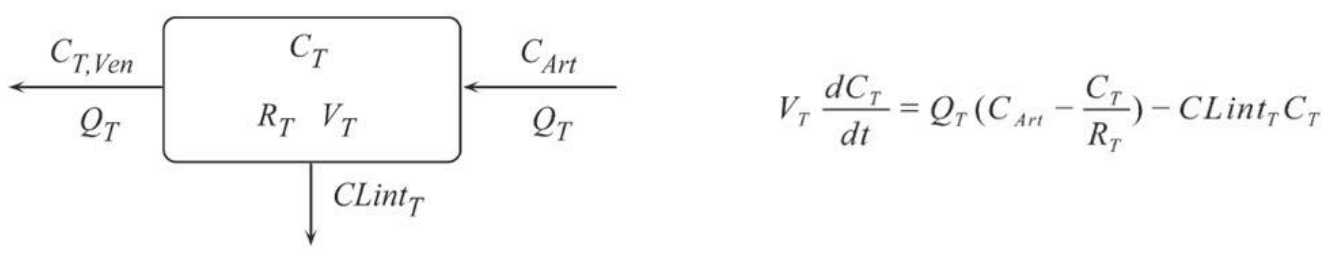

D Diffusion limited model for eliminating organs

\begin{tabular}{|c|c|c|c|}
\hline$C_{T, V e n}$ & $k_{V, E V} C_{T, V} \quad V_{T, V}$ & $C_{A r t}$ & \multirow{2}{*}{$\begin{array}{l}V_{T, V} \frac{d C_{T, V}}{d t}=Q_{T}\left(C_{A r t}-C_{T, V}\right)-k_{V, E V} f u_{b} C_{T, V} V_{T, V}+k_{E V, V} C_{T, E V} V_{T, E V} \\
V_{T, E V} \frac{d C_{T, E V}}{d t}=k_{V, E V} f u_{b} C_{T, V} V_{T, V}-k_{E V, V} C_{T, E V} V_{T, E V}-\operatorname{CLint}_{T} C_{T, E V}\end{array}$} \\
\hline$Q_{T}$ & $\checkmark{ }_{k_{E V, V}} C_{T, E V} V_{T, E V}$ & $Q_{T}$ & \\
\hline & & & \\
\hline
\end{tabular}


certain tissue, if the rate limiting step is assumed to happen in the capillary membrane, then the two compartments represent the vascular and extra-vascular compartments; if the rate limiting step is assumed to happen at the cell membrane, then the two compartments represent intra- and extra-cellular compartments. For the three compartment model in a certain tissue, it is assumed that both the capillary and cell membrane are rate-limiting steps for the drug. And the three compartments represent vascular, interstitial, and cellular compartments [116]. For eliminating organs, an eliminating function component is added to the model structures (Figure 1-9C, D) [168]. In reality, a whole body PBPK model can be composed of both perfusion- and diffusion-limited tissues. For example, the whole body PBPK model developed for 17-(allylamino)-17-demethoxygeldanamycin (17-AAG) contains both perfusion-limited organs (lung, brain, heart, spleen, kidney, and muscle) and diffusion-limited tissue (tumor) [168]. Whole body PBPK model developed for topotecan contains both perfusion-limited organs (lung, heart, muscle, adipose, skin, brain, liver, spleen, and gut) and diffusion-limited organs (kidney and testes) [170].

1.3.2.3. Writing differential equations. As in classic PK modeling, PBPK modeling equations are written following the mass balance equation. The difference is that the structure and components of the model represent real physiology. Examples of the equations are listed in Figure 1-9 [168]. During model development process, equations may be modified.

1.3.2.4. Estimating parameters. In order to estimate parameters, in addition to experimental data, physiological and anatomical data should be collected for modeling. However, obtaining reliable physiological parameters is not an easy task. Inaccurate physiological parameters used in the model will affect value of the final model. Among all the parameters, sensitivity analysis showed that blood flow is the most influential parameter [171]. Since various conditions such as anesthesia and stress can alter blood flow, using physiological data that are obtained under these conditions may bias the modeling results.

Because of the complexity of the PBPK model, PBPK parameter estimation usually is conducted from the open loop to closed loop (Figure 1-10). Blood concentration is first modeled using the empirical approach. The established blood PK model from the empirical approach is then used as a forcing function to each individual tissue. In this step, information such as blood flow, organ weight, and partition coefficient are used or estimated. Since individual tissues are still isolated, the model at this stage is open loop. Then the PBPK model is modified to a closed circulating system. Due to the computational challenges in modeling all the PBPK parameters at the same time, an open-loop approach followed by closed-loop approach is recommended [117]. 
Open Loop

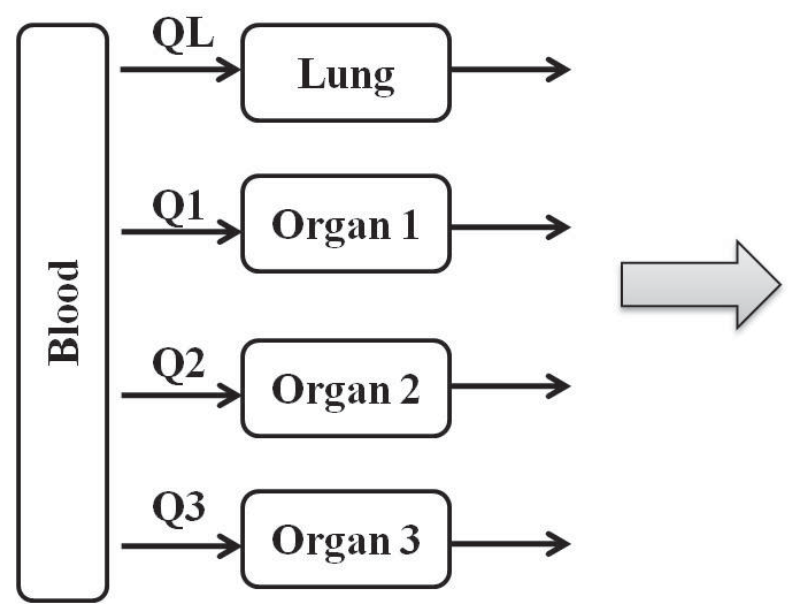

Closed Loop

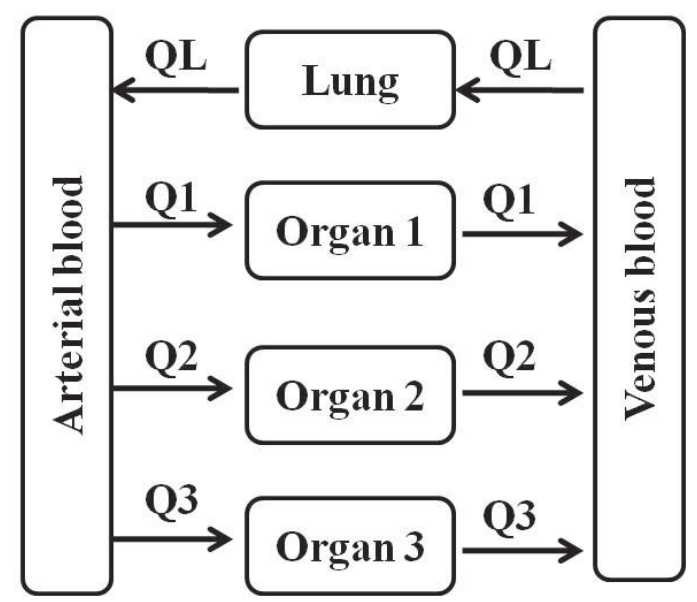

Figure 1-10. Schematic structure of open loop and closed loop

In the open loop form, separate PBPK models were established for individual organs. Later in the closed loop form, all the modeled organs are linked together to form an anatomically integrated PBPK model. Q refers to blood flow to the lung (QL), organ 1 $(\mathrm{Q} 1)$, organ $2(\mathrm{Q} 2)$, and organ $3(\mathrm{Q} 3)$. Input can be any site of the body. 


\subsubsection{Applications of PBPK model in drug discovery and development}

\subsubsection{Predicting human ADME and whole plasma PK profile using PBPK}

model. In early drug development stages, the measurements or simulation of drug-specific data - such as molecular weight, Log P (or Log D), pKa, plasma protein binding, and in vitro hepatic intrinsic clearance - can be incorporated into the PBPK model to predict animal and human ADME and facilitate candidate selection. Full plasma concentration-time profiles following different dosing schedules can be predicted by the PBPK models. For example, using the PBPK model, Jones et al. from F. Hoffmann-La Roche reported better prediction of human plasma concentration compared to commonly used allometric scaling (Dedrick approach) for compounds with distinct physicochemical and pharmacokinetic properties $[119,172]$. In addition to the report from Jones et al., various approaches had been reported to predict ADME [119, 125, 173-180]. These prediction methods are generally complex, and the new trend and preferred approach is to perform prediction using commercially available PBPK software such as Simcyp, Gastroplus, and PK-Sim (mentioned in section 1.3.1.3).

Figure 1-11 represents the general scheme for predicting human PK data using this approach. Human concentration-time profiles under various dosing schedules can be generated when drug-specific data are provided. This can greatly simplify the ADME prediction process. In addition, animal PK data can be incorporated into the PBPK model to generate a human PK profile or to validate the IVIVE prediction. In this case, a PK parameter (such as clearance) estimated using the classic PK model is incorporated into the PBPK model to obtain a better prediction. More studies are needed to validate the predictive ability of the software. And more improvement is needed, especially for predicting oral absorption and for dealing with complex transporters involved in the PK process.

1.3.3.2. Drug distribution in target tissues and tumors. PBPK models have been used to understand and predict target tissue and tumor concentrations. In the oncology area, a PubMed search (09/12/2011) using “physiologically based pharmacokinetic model cancer" or "physiologically based pharmacokinetic model tumor" returned 153 papers. PBPK models had been used for antibody [155-158, 160-163, 181-183], liposomal [184], imaging agents [185-187], radioimmunodetection and radioimmunotherapy [188, 189], and small molecules such as topotecan [170], docetaxel [190, 191], gefitinib [192-194], moxifloxacin [195], capecitabine [196, 197], temozolomide [198, 199], genistein [200, 201], doxorubicin [136], 17-AAG [168], and methotrexate $[202,203]$. General methods and principles of model development have been discussed in 1.3.1 and 1.3.2.

Partition coefficient is an important parameter in the PBPK model. Multiple methods for obtaining the partition coefficient values were applied as follows: 1. Estimation using PBPK model. 2. Estimation using non-compartmental modeling. Shah et al. reported a whole body PBPK model for topotecan [170]. In that model, a tissue to 


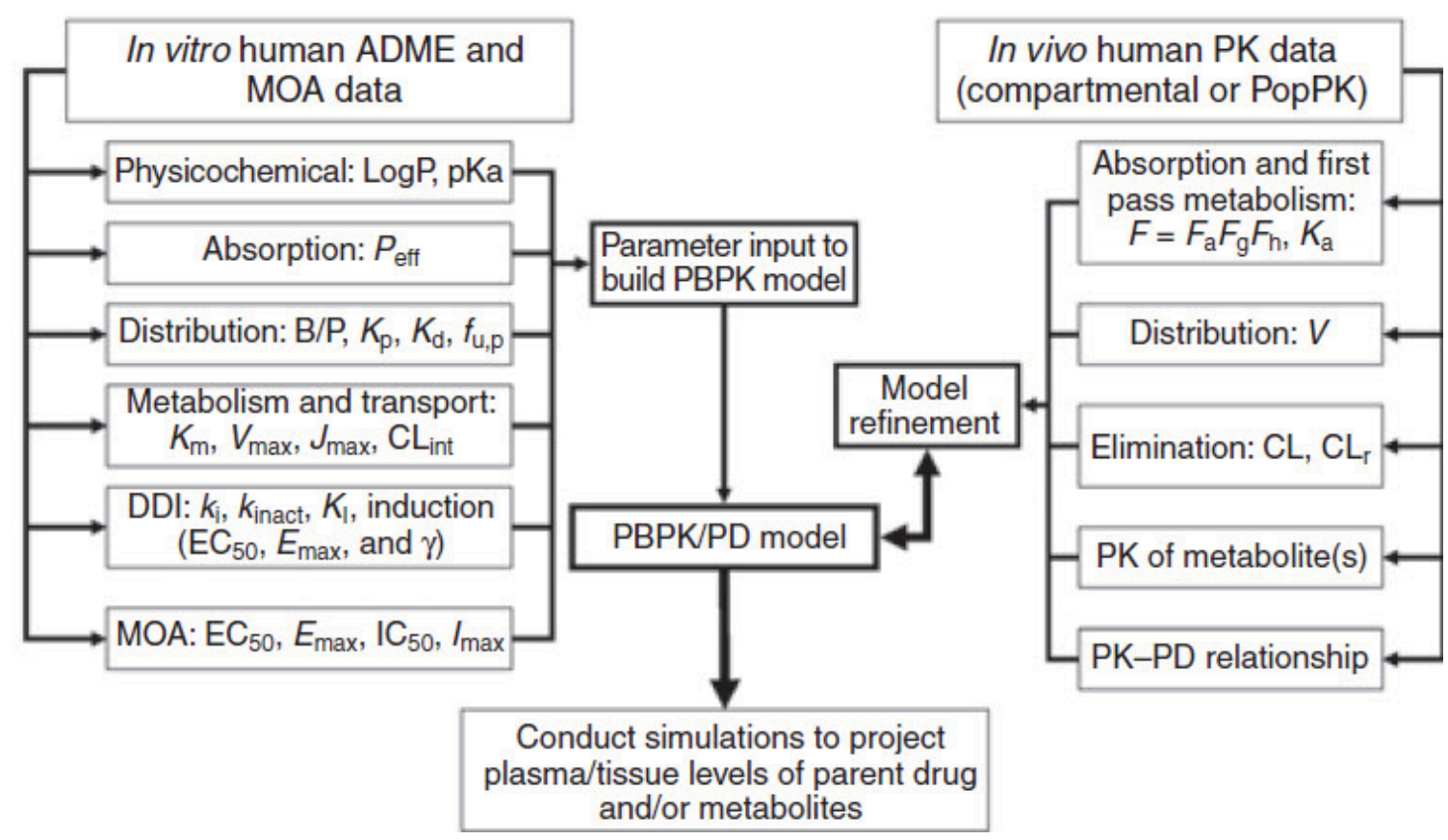

Figure 1-11. General schemes to incorporate drug dependent parameters into a PBPK model

Peff, jejunum permeability; AUC, area under the concentration vs. time curve; B/P, blood to plasma ratio; Cmax, maximum concentration; CL, clearance; CLint, intrinsic clearance; CLr, renal clearance; DDI, drug-drug interaction; $\mathrm{EC}_{50}$ or $\mathrm{IC}_{50}$, concentration causing half of the maximal effect of induction or inhibition; Emax or Imax, maximum effect of induction or inhibition; F, bioavailability; Fa, fraction absorbed; Fg, bioavailability in the gut; Fh, bioavailability in the liver; fu,p, unbound fraction in plasma; $\gamma$, Hill coefficient; Jmax, maximum rate of transporter-mediated efflux/uptake; $\mathrm{Ka}$, first-order absorption rate constant; $\mathrm{Kd}$, dissociation constant of drug-protein complex; Ki, reversible inhibition constant; KI, apparent inactivation constant, concentration causing half of the maximal inactivation; kinact, apparent maximum inactivation rate constant; Km, Michaelis-Menten constant, substrate concentration causing half of the maximal reaction or transport; Kp, tissue-to-plasma partition coefficient; LogP, logarithm of the octanol-water partition coefficient; MOA, mechanism of action; PD, pharmacodynamics; PK, pharmacokinetics; PopPK, population pharmacokinetics; V, volume of distribution; Vmax, maximum rate of metabolite formation.

Source: Reprinted with permission. Zhao, P., et al., Applications of physiologically based pharmacokinetic (PBPK) modeling and simulation during regulatory review. Clinical Pharmacology and Therapeutics, 2011. 89(2): p. 259-67. 
plasma partition coefficient for each tissue was first estimated using the ratio of AUC (estimated from non-compartmental modeling) for the tissue to the ratio in plasma. These values were then fixed and applied to the whole body PBPK model. 3. In vitro measurements. Bradshaw-Pierce et al. reported a PBPK model for docetaxel [204]. The PBPK model for docetaxel was developed incorporating specific binding of docetaxel to intracellular components, liver metabolism, biliary elimination, and fecal and urinary excretion. Tissue/blood partition coefficients were determined in vitro [204, 205]. Docetaxel was incubated with minced tissue at $37^{\circ} \mathrm{C}$ with gentle shaking for $24 \mathrm{~h}$. Samples were centrifuged to separate tissue from saline. Docetaxel concentration was measured in both the saline and tissue layer. The partition coefficient was determined by the ratio of docetaxel concentrations in the tissue layer to the saline layer. These values were fixed and applied to the whole body PBPK model. 4. Calculation. Sung et al. reported a PBPKPD model for UTF (5-FU, tegafur, and uracil) [206]. Partition coefficients for tissues were calculated based on the n-octanol-water partition coefficient developed by Poulin and Theil [207, 208]. The estimation of partition coefficient for tumor was adjusted during model development. Once a PBPK model is established, scaling the concentration from one species to another can be done by changing the physiological information but keeping the model structure and assuming identical partition coefficients between species [168, 192, 198].

1.3.3.3. Predicting the risk for drug-drug interactions. Drug-drug interactions can cause serious toxicities or loss of efficacy. Due to the fact that many drugs are substrates and/or inhibitors of various drug metabolizing enzymes and efflux transporters, the degree of drug-drug interaction is a concern when drugs are used in combination. To understand the potential for a drug-drug interaction for a certain drug, evaluations are usually conducted in clinical trials or in preclinical settings. Now, by combining the physiological information and the drug metabolism enzyme/transporter inhibition/or induction knowledge obtained in vitro experimentally, the PBPK model can be used to simulate and predict drug-drug interaction in humans. Multiple PBPK model applications for predicting drug-drug interactions have been reported [130, 143-150]. For example, Johnson et al. built a pediatric PBPK (P-PBPK) model to predict drug concentration-time profiles and drug-drug interactions in children [130]. Using Simcyp Pediatric ADME Simulator, concentration time profile was simulated for a 2-year-old child prescribed with CYP3A4 inducer, CYP3A4 inhibitor, and substrates with complex dosing schedules.

The FDA reported example cases of using a PBPK model during investigational new drugs (INDs) and new drug applications (NDAs) reviews between July 2008 and June 2010 [149]. In these cases, the PBPK approach had been used to address multiple specific regulatory questions, such as whether a drug-drug interaction study can be ruled out even though the ratio of in vivo inhibitor concentration I (maximum plasma concentration at the highest proposed dose) to the in vitro inhibition constant (Ki) (I/Ki) is $>0.1$. The software used in the study included GetData 2.24 (for digitizing concentration-vs.-time profiles), WinNonlin 5.2, NONMEM (for PK parameter estimation), and PBPK simulators (Simcyp, PK-Sim, Gastroplus, and SAAMII). At the same time, it should be mentioned that the PBPK model used in this case relies largely on 
in vitro and in silico data. Some important parameters needed may not be available in earlier drug development stage. As the compound progresses to later stage, the PBPK model can be refined incorporating the available data.

\subsubsection{Limitation of PBPK modeling}

So far, in comparison to the classical PK model, the application of the PBPK approach is still limited despite significant potential. Developing a PBPK model is demanding because of the investment of large amount of time and effort to obtain information needed to establish the model. In addition, data required to establish the model are not always available. Variations in physiological parameters obtained from the literature, our current limited knowledge about the underlying mechanism about drug ADME process, and wrong assumptions can all affect the quality of the model. The PBPK model is usually structurally complex and much more methodologically and computationally challenging compared to the classic PK model. Furthermore, PBPK model is not as mature as conventional PK model.

Despite all of the limitations of the PBPK model mentioned above, the PBPK area is developing rapidly. The knowledge and methods for building the model are continuously accumulating and improving. The PBPK model breaks the limit of conventional PK by building a model and understanding the PK process based on the real physiological and anatomical system, and by incorporating rich knowledge learned over the years about effect of drug dependent and independent factors on PK. In the drug discovery and development area, pharmaceutical companies are acquiring PBPK modeling software for predicting human $\mathrm{PK}$ based on in vitro and/or animal in vivo PK data. Also, the regulatory authority has started appreciating the benefit of the PBPK model and applying the PBPK model to address drug regulatory review questions. At last, but not least, the PBPK model will be applied with increased frequency in the toxicology and oncology areas where understanding target tissue or tumor concentration is important yet data are usually inaccessible in the human.

\subsection{Summary}

Reactivating the p53 pathway is regarded as an appealing nongenotoxic approach for treating tumors with wild-type p53. Unlike adult cancers, pediatric malignancies usually retain a high percentage of wild-type p53 status at diagnosis. Using neuroblastoma as an example, $98 \%$ of neuroblastoma tumors retain wild-type p53 at diagnosis [209, 210]. Even in relapse neuroblastoma, a majority of the tumors are still p53 wild-type [211]. Nutlin-3a is a small molecule inhibitor that targets the MDM2/MDMX-p53 interaction. It is currently under pre-clinical investigation in a variety of solid tumor and leukemia models, and has shown promising in vitro and in vivo activities. Like most other anticancer drugs, it is most likely that nutlin-3 will be used in combination. Studies have suggested synergistic effects when nutlin-3 and other chemotherapeutic drugs were co-administered, independent of p53 status, by enhancing 
the ability of anticancer drugs to activate apoptosis or by reversing P-gp-mediated drug resistance. $\mathrm{ABC}$ transporters play important roles in drug resistance and ADME processes. The effect of nutlin-3a on BCRP had not been reported before.

Understanding the pharmacokinetics of a compound is critical in preclinical drug development. An understanding of the systemic disposition of nutlin-3a, as well as the distribution to target tissue or tumor sites, will provide a rational basis for the selection of dosage regimens for preclinical models. To date, the pharmacokinetics of nutlin-3a has not been reported. Whole-body PBPK model, which is based on anatomical compartments and blood flow, is an excellent tool to describe and predict drug concentration not only in blood but also in target tissues.

\subsection{Specific Aims}

The objective of specific aim 1 was to assess the effect of nutlin-3a on cell viability, both as a single agent and in combination with one of the commonly used anti-neuroblastoma agents, topotecan.

The objective of specific aim 2 was to investigate whether nutlin-3a inhibits BCRP, thus sensitizing cells to enhanced killing by anti-cancer drugs that are BCRP substrates. Based on initial observations, we performed a series of studies to investigate the effect of nutlin-3a treatment on BCRP expression and function comprehensively [212].

The objective of specific aim 3 was to perform pharmacokinetic studies and develop a PBPK model describing the disposition of nutlin-3a in plasma and tissues, including adipose, adrenal gland, bone marrow, brain, liver, lung, intestine, muscle, retina, spleen, and vitreous fluid. The PBPK model was used to perform simulations, which - in combination with in vitro cell sensitivity data - provided rationale for choosing dosing regimens for mouse models of common childhood cancers, including retinoblastoma, neuroblastoma, rhabdomyosarcoma, and acute lymphoblastic leukemia (ALL) [213]. 


\section{CHAPTER 2. MDM2 ANTAGONIST NUTLIN-3A SYNERGISTICALLY INHIBITS NEUROBLASTOMA CELL GROWTH WITH TOPOTECAN}

\subsection{Introduction}

Neuroblastoma [214] is an embryonic malignancy of the sympathetic nervous system. It is the second most common extra-cranial solid tumor of childhood [215] and the most common cancer of infancy [216]. While other childhood cancers have experienced an improved cure rate over the past decades, high risk neuroblastoma is still one of the most difficult tumors to treat, with less than 30\% long-term survival despite aggressive multi-modal therapy including surgery, radiation, high-dose chemotherapy, and stem cell transplantation [217].

To provide a new therapy for neuroblastoma, effective molecular targets should be selected. One of the targets that we studied is p53. p53 plays a central role in regulating cell cycle arrest, senescence, and apoptosis. The p53 gene is mutated in half of all tumors, and in those tumors that retain wild-type p53, p53 function may also be suppressed by its negative regulators. As a key negative regulator of p53 [218], oncogene MDM2 effectively impairs p53 stability and activity. Strong evidence for the relationship between MDM2 and p53 has made inhibition of the MDM2-p53 interaction an attractive target for reactivating $\mathrm{p} 53$.

One crucial point of neuroblastoma is that $98 \%$ of neuroblastoma tumors retain wild-type p53 at diagnosis [209, 210]. Even in relapse neuroblastoma, a majority of the tumors are still p53 wild-type [211]. Although many mechanisms for inactivation of p53 pathway had been proposed [211,219], several studies indicate that the p53 downstream pathway is intact in neuroblastoma and can be activated in response to DNA damage [220]. These data suggest that regardless of many other unclear possible mechanisms of p53 inactivation, p53 downstream pathway is intact, which encouraged us to restore the function of p53 by directly stabilizing p53 protein using the MDM2-p53 interaction inhibitor to treat neuroblastoma.

The results of preclinical studies have shown that the MDM2 antagonist nutlin-3a is a promising agent to reactivate $\mathrm{p} 53$. Nutlin-3a has demonstrated anti-tumor efficacy in preclinical models of human osteosarcoma, retinoblastoma, and KSHV lymphoma with wild-type p53 [17, 24, 25, 39-41]. In neuroblastoma, studies have found that MDM2 antagonist nutlin-3a is effective both in vitro [36, 221, 222] and in vivo [43].

Several studies suggested that when nutlin-3a was combined with anti-cancer agents, synergistic growth inhibition was observed [31, 35, 37]. In neuroblastoma, nutlin-3 synergized (or sensitized) neuroblastoma cells to chemotherapeutic agents (R)-roscovitine [36], cisplatin [221], etoposide [221], and doxorubicin [223, 224]. So far, combining nutlin-3 with topotecan, a commonly used anti-neuroblastoma agent, has not been reported in neuroblastoma research. 
In this study, we reported that nutlin-3a synergistically inhibited neuroblastoma cell growth with topotecan. To explore a possible mechanism, we studied the effect of nutlin-3a on the expression of efflux transporter P-gp.

\subsection{Materials and Methods}

\subsubsection{Reagents}

Nutlin-3a were synthesized in the Department of Chemical Biology at St. Jude Children's Research Hospital, Memphis, TN, and were solubilized in dimethyl sulfoxide (DMSO) (ATCC, Manassas, VA) to a final concentration of $20 \mathrm{mM}$. Topotecan hydrochloride was purchased from GlaxoSmithKline (Philadelphia, PA) and solubilized to a final concentration of $20 \mathrm{mM}$ in sterile water. CellTiter $96{ }^{\circledR}$ AQueous MTS assay reagents were purchased from Promega (Madison, WI).

\subsubsection{Cell culture}

Neuroblastoma cell lines NB1691, NB1643, NBEBC1, SHSY-5Y, SKNAS, and SKNBE2C were cultured in antibiotic-free RPMI-1640 medium (Cellgro $\AA$, Manassas, VA) containing $10 \%$ fetal bovine serum (FBS) (Invitrogen, Carlsbad, CA) and 1\% L-Glutamine (Invitrogen, Carlsbad, $\mathrm{CA}$ ) at $37^{\circ} \mathrm{C}$ in $5 \% \mathrm{CO}_{2}$.

\subsubsection{Cell viability assay}

Briefly, cells were seeded in $100 \mu \mathrm{l}$ phenol red-free RPMI-1640 medium (Cellgro ${ }^{\circledR}$, Manassas, VA) in 96-well plates and allowed to attach overnight. Cells were treated with increasing concentrations of nutlin-3a alone or increasing concentrations of topotecan in combination with nutlin-3a for 6 hours. After 6 hours, topotecan was washed off and cells were incubated with or without nutlin-3a for additional 18 hours. Cell viabilities were tested by MTT assay or CellTiter 96® AQueous MTS assay following the manufacturer's protocol. $\mathrm{IC}_{50}$ values were calculated using ADAPT 5 (Biomedical Simulations Resources, Los Angeles, CA) [225].

\subsubsection{Synergy study}

Synergism, additive effects, and antagonism were assessed using the median effect method developed by Chou [226]. The combination index (CI) values at non-fixed nutlin-3a/topotecan concentration ratios were calculated using the commercially available software Calcusyn 2.1 (Biosoft, Cambridge, United Kingdom). CI values $<1.0$ indicate synergism, $\mathrm{CI}$ values $=1.0$ indicate additive effect, and CI values $>1.0$ indicate antagonism [226]. 


\subsection{5. qRT-PCR}

Cells were treated with $10 \mu \mathrm{M}$ nutlin-3a for 24 hours. Total RNA samples were extracted using Qiagen RNeasy ${ }^{\circledR}$ Plus Mini Kit (Valencia, CA) following manufacturer's instructions. RNA concentrations were quantified using Beckman DU-600 spectrophotometer (Beckman Instruments Inc., Fullerton, CA). Genomic DNA elimination, RT reaction, and cDNA synthesis reaction were performed using the $\mathrm{RT}^{2}$ First Strand Kit (SABiosciences, Frederick, MD) following the manufacturer's protocol.

Primers used for RT-PCR analysis were synthesized by the Hartwell Center at St. Jude. Children's Research Hospital. For MDR1, the forward primer sequence was CCATCATTGCAATAGCAGG, and the reverse primer sequence was TGTTCAAACTTCTGCTCCTGA. For GAPDH control, the forward primer sequence was AAGGACTCATGACCACAGTCCAT, and the reverse primer sequence was CCATCACGCCACAGTTTCC. SYBR Green qPCR Master Mix was purchased from SABiosciences (Frederick, MD). QRT-PCR reactions were performed on ABI Prism ${ }^{\circledR}$ 7700 Sequence Detection System (Applied Biosystems, Foster City, CA). The reactions were conducted under the following conditions:

$\begin{array}{lll}15 \mathrm{~min} & 95^{\circ} \mathrm{C} & 1 \mathrm{cycle} \\ 18 \mathrm{sec} & 95^{\circ} \mathrm{C} & 40 \mathrm{cycles} \\ 30 \mathrm{sec} & 52^{\circ} \mathrm{C} & \\ & & \\ 15 \mathrm{sec} & 95^{\circ} \mathrm{C} & \\ 15 \mathrm{sec} & 60^{\circ} \mathrm{C} & 1 \mathrm{cycle} \\ 15 \mathrm{sec} & 95^{\circ} \mathrm{C} & \end{array}$

\subsubsection{Western blots}

Cells were lysed in M-PER Mammalian Protein Extraction Reagent (Thermo Scientific, Rockford, IL) following manufacturer's instructions. Protein concentrations were determined using the BCA protein assay (Thermo Scientific Rockford, IL). 8-16 $\mu \mathrm{g}$ of proteins were resolved by SDS PAGE gels (4-12\% gradient Bis/Tris NuPage gels) (Invitrogen, Carlsbad, CA) with MOPS SDS running buffer (Invitrogen, Carlsbad, CA) before transferring onto $0.45 \mu \mathrm{m}$ pore size InvitrolonTM PVDF membranes (Invitrogen, Carlsbad, CA). P-gp was detected using the mouse monoclonal antibody clone C-219 (Alexis Biochemicals, San Diego, CA). $\beta$-actin (AC-15, Sigma-Aldrich, St. Louis, MO) was used as the loading control. 


\subsubsection{Statistical analysis}

All data were expressed as mean \pm standard deviation. Data were analyzed for statistical significance using Student's t-test. Differences with $\mathrm{p}<0.05$ were considered statistically significant.

\subsection{Results}

\subsubsection{Effect of single agent nutlin-3a on the cell viability of neuroblastoma cell lines}

p53 wild-type NB1691, NB1643 and p53 mutant SKNBE2C cells were incubated with increasing concentrations of nutlin-3a alone for 24 hours. $\mathrm{IC}_{50}$ values were determined by MTT assay (Figure 2-1). The $\mathrm{IC}_{50}$ values of nutlin-3a in $\mathrm{p} 53$ wild-type cells NB1691 and NB1643 were $3.99( \pm 0.12) \mu \mathrm{M}$ and $4.29( \pm 0.20) \mu \mathrm{M}$, respectively. p53 mutant SKNBE2C cells were resistant to nutlin-3a treatment, with $\mathrm{IC}_{50}$ value of 26 $( \pm 295.62) \mu \mathrm{M}$.

\subsubsection{Combination of nutlin-3a with topotecan in NB1691 cells}

NB1691 cells were incubated with increasing concentrations of topotecan in combination with DMSO control or $2 \mu \mathrm{M}$ nutlin-3a for 6 hours. After 6 hours, cells were washed with drug-free media. Media containing either DMSO control or $2 \mu \mathrm{M}$ nutlin-3a was added back to the wells for additional incubation of 18 hours. $\mathrm{IC}_{50}$ values were determined by MTT assay (Table 2-1). Without combination, the $\mathrm{IC}_{50}$ value of topotecan in NB1691 cells was $8.39( \pm 1.04) \mu \mathrm{M}$. Combination of topotecan with nutlin-3a resulted in a 4.7 -fold reduction of $\mathrm{IC}_{50}$ to $1.79( \pm 0.29) \mu \mathrm{M}$.

\subsubsection{Synergistic effect of nutlin-3a in combination with topotecan}

To study the interaction of nutlin-3a and topotecan in NB1691 cells, NB1691 cells were incubated with various concentrations of nutlin-3a and topotecan. Cells were treated with nutlin-3a and topotecan for 6 hours. After 6 hours, cells were washed with drug-free media. Media containing various concentrations of nutlin-3a was added back to the wells for additional incubation of 18 hours. $\mathrm{IC}_{50}$ values were determined by MTS assay.

The CI values at non-fixed nutlin-3a/topotecan concentration ratios were calculated using Calcusyn 2.1. The calculated CI values were listed in Table 2-2. Fractional effect is defined as the fraction of cells affected by nutlin-3a and topotecan combination. A fractional effect value of 0 indicates no inhibition, and a fractional effect value of 1 indicates $100 \%$ inhibition of cell viability. When topotecan concentrations were less than $25 \mu \mathrm{M}$, CI values were generally $<1$, indicating a synergistic effect. When 


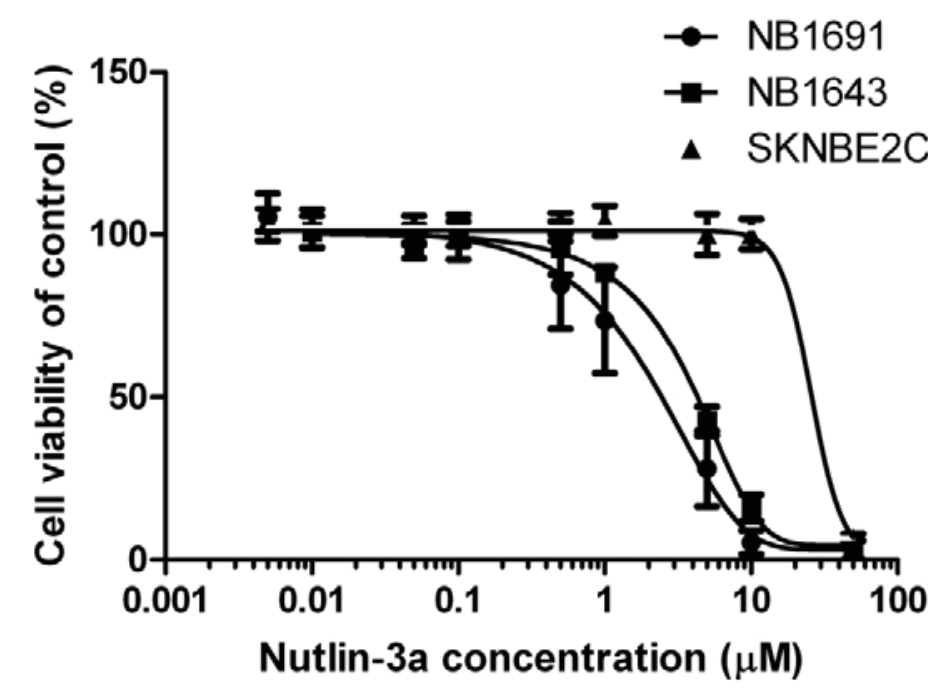

Figure 2-1. Effect of single agent nutlin-3a on the cell viability of neuroblastoma cell lines

p53 wild-type NB1691, NB1643 and p53 mutant SKNBE2C cells were incubated with increasing concentrations of nutlin-3a alone for 24 hours. $\mathrm{IC}_{50}$ values were determined by MTT assay in 5 replicates. $\mathrm{IC}_{50}$ values were calculated using ADAPT 5. Values are presented as mean $\pm \mathrm{SD}$. Data are representative of one experiment.

Table 2-1. Combination of nutlin-3a with topotecan in NB1691 cells

\begin{tabular}{cccc}
\hline Treatment & $\begin{array}{c}\mathrm{IC}_{50} \\
(\mu \mathrm{M})\end{array}$ & $\mathrm{SD}$ & $\begin{array}{c}\text { Confidence interval }(95 \%) \\
(\mu \mathrm{M})\end{array}$ \\
\hline Topotecan & 8.39 & 1.04 & {$[6.32,10.46]$} \\
Nutlin-3a +topotecan & 1.79 & 0.29 & {$[1.21,2.38]$} \\
\hline
\end{tabular}

NB1691 cells were incubated with increasing concentrations of topotecan in combination with DMSO control or $2 \mu \mathrm{M}$ nutlin-3a for 6 hours. After 6 hours, cells were washed with media. Media containing either DMSO control or $2 \mu \mathrm{M}$ nutlin-3a was added back to the wells for additional incubation of 18 hours. IC $_{50}$ values were determined by MTT assay. Combination of topotecan with nutlin-3a resulted in a 4.7-fold reduction of $\mathrm{IC}_{50}$ to from $8.39( \pm 1.04) \mu \mathrm{M}$ to $1.79( \pm 0.29) \mu \mathrm{M}$. $\mathrm{IC}_{50}$ values were calculated using ADAPT 5. Data are representative of one experiment. 
Table 2-2. Synergistic effect of nutlin-3a in combination with topotecan

\begin{tabular}{|c|c|c|c|}
\hline Topotecan $(\mu \mathrm{M})$ & Nutlin-3a $(\mu \mathrm{M})$ & $\mathrm{Fa}$ & Combination index (CI) \\
\hline 0.39 & 1.25 & 0.46 & 0.39 \\
\hline 0.39 & 2.50 & 0.46 & 0.64 \\
\hline 0.39 & 5.00 & 0.46 & 1.15 \\
\hline 0.39 & 10.00 & 0.68 & 0.71 \\
\hline 0.39 & 20.00 & 0.67 & 1.56 \\
\hline 0.78 & 1.25 & 0.47 & 0.46 \\
\hline 0.78 & 2.50 & 0.47 & 0.70 \\
\hline 0.78 & 5.00 & 0.47 & 1.17 \\
\hline 0.78 & 10.00 & 0.72 & 0.60 \\
\hline 0.78 & 20.00 & 0.74 & 1.05 \\
\hline 1.56 & 1.25 & 0.59 & 0.26 \\
\hline 1.56 & 2.50 & 0.59 & 0.40 \\
\hline 1.56 & 5.00 & 0.59 & 0.68 \\
\hline 1.56 & 10.00 & 0.75 & 0.50 \\
\hline 1.56 & 20.00 & 0.75 & 1.00 \\
\hline 3.13 & 1.25 & 0.67 & 0.19 \\
\hline 3.13 & 2.50 & 0.67 & 0.28 \\
\hline 3.13 & 5.00 & 0.67 & 0.47 \\
\hline 3.13 & 10.00 & 0.77 & 0.44 \\
\hline 3.13 & 20.00 & 0.77 & 0.88 \\
\hline 6.25 & 1.25 & 0.72 & 0.17 \\
\hline 6.25 & 2.50 & 0.72 & 0.24 \\
\hline 6.25 & 5.00 & 0.72 & 0.39 \\
\hline 6.25 & 10.00 & 0.77 & 0.47 \\
\hline 6.25 & 20.00 & 0.78 & 0.87 \\
\hline 12.50 & 1.25 & 0.68 & 0.40 \\
\hline 12.50 & 2.50 & 0.68 & 0.49 \\
\hline 12.50 & 5.00 & 0.68 & 0.67 \\
\hline 12.50 & 10.00 & 0.71 & 0.79 \\
\hline 12.50 & 20.00 & 0.73 & 1.23 \\
\hline 25.00 & 1.25 & 0.68 & 0.73 \\
\hline 25.00 & 2.50 & 0.68 & 0.82 \\
\hline 25.00 & 5.00 & 0.68 & 1.01 \\
\hline 25.00 & 10.00 & 0.64 & 1.96 \\
\hline 25.00 & 20.00 & 0.63 & 3.06 \\
\hline 50.00 & 1.25 & 0.55 & 6.39 \\
\hline 50.00 & 2.50 & 0.55 & 6.56 \\
\hline 50.00 & 5.00 & 0.55 & 6.89 \\
\hline 50.00 & 10.00 & 0.59 & 4.71 \\
\hline 50.00 & 20.00 & 0.75 & 1.46 \\
\hline
\end{tabular}


Table 2-2. (Continued)

\begin{tabular}{cccc}
\hline Topotecan $(\mu \mathrm{M})$ & Nutlin-3a $(\mu \mathrm{M})$ & $\mathrm{Fa}$ & Combination index $(\mathrm{CI})$ \\
\hline 100.00 & 1.25 & 0.63 & 4.91 \\
100.00 & 2.50 & 0.63 & 5.03 \\
100.00 & 5.00 & 0.63 & 5.26 \\
100.00 & 10.00 & 0.62 & 6.34 \\
100.00 & 20.00 & 0.64 & 5.87 \\
\hline
\end{tabular}

NB1691 cells were incubated with various concentrations of nutlin-3a and topotecan. $\mathrm{IC}_{50}$ values were determined by MTS assay. The CI values at non-fixed nutlin-3a/topotecan concentration ratios were calculated using commercially available software Calcusyn 2.1. CI values $<1.0$ indicate synergism, CI values $=1.0$ indicate additive effect and $\mathrm{CI}$ values $>1.0$ indicate antagonism. Data are representative of one experiment. 
topotecan concentrations were higher than $25 \mu \mathrm{M}$, CI values were $>1$, indicating antagonism.

\subsubsection{MDR1 gene expression change after nutlin-3a treatment}

Since wild-type p53 was reported to repress the MDR1 promoter [97-99], the synergism observed in NB1691 may be explained by the reduction of MDR 1 gene expression. To evaluate the effect of nutlin-3a on the MDR1 gene expression, we performed qRT-PCR in a panel of neuroblastoma cell lines after $10 \mu \mathrm{M}$ nutlin-3a treatment for 24 hours. Compared to control cells, a 3-fold reduction of MDR1 gene expression was observed in MDM2-amplified NB1691 cells. In contrast, a 1.54-fold increase of $M D R 1$ gene expression was observed in p53 mutant SKNBE2C cells. There were no changes in the gene expression of MDM2 non-amplified p53 wild-type cells SHSY5Y, NB1643, and NBEBC1 cells and p53 null SKNAS cells. Results in Figure 2-2 represent pooled data of three independent experiments.

\subsubsection{P-gp protein expression after nutlin-3a treatment}

In addition to evaluating $M D R 1$ expression at the message level, P-gp expression at the protein level was evaluated using western blot. Results in Figure 2-3 suggest reduced protein expression of P-gp in NB1691 and NBEBC1 cells. No increased P-gp protein expression was observed in SKNBE2C cells.

\subsection{Discussion}

Our preliminary studies showed that targeting MDM2-p53 interaction using nutlin-3a reduced cell growth in neuroblastoma cells. p53 wild-type cells were much more sensitive to nutlin-3a treatment compared to p53 mutant cells. When nutlin-3a was combined with topotecan, a synergistic effect was observed when topotecan concentrations were $<25 \mu \mathrm{M}$. When topotecan concentrations were $>25 \mu \mathrm{M}$, the nutlin-3a and topotecan combination caused antagonistic effect. This is the first study looking at nutlin-3a and topotecan interaction in neuroblastoma cell lines. Synergism has been reported in other tumor types, such as retinoblastoma [24] and intraocular melanoma [227], when nutlin-3a and topotecan were combined. Topotecan concentrations tested in those two studies did not include levels $>25 \mu \mathrm{M}$.

To investigate possible mechanism of synergy, we performed qRT-PCR and western blot and found reduced MDRl expression at both the message level and protein level in MDM2-amplified NB1691 cells. In MDM2 non-amplified NBEBC1 cells, although no MDRl gene expression change was observed, western blot data showed reduced expression of $\mathrm{P}$-gp protein. Since topotecan is a substrate of P-gp, synergism observed may be partly explained by reduced P-gp expression. However, it should be 


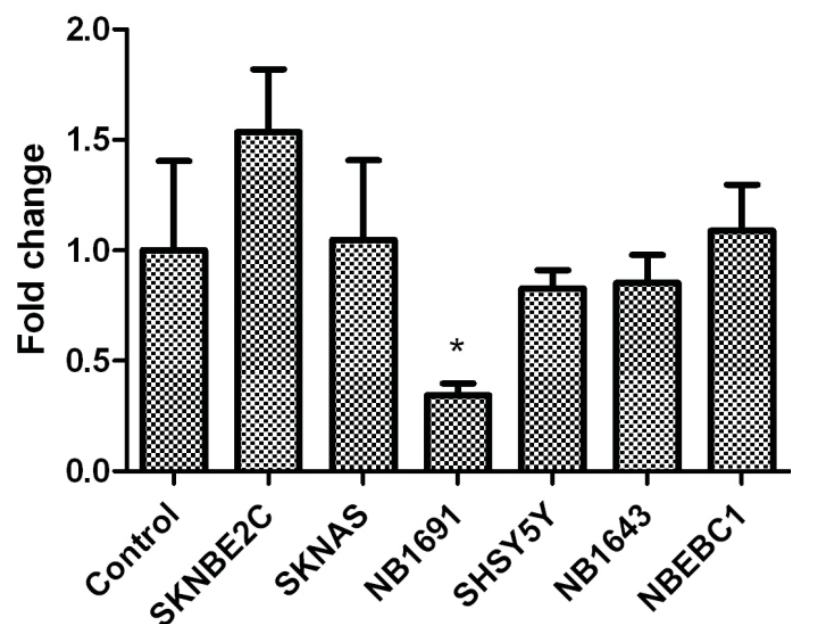

Figure 2-2. $\quad M D R 1$ gene expression change after nutlin-3a treatment

MDR1 gene expression changes after $10 \mu \mathrm{M}$ nutlin-3a treatments were evaluated using qRT-PCR in a panel of neuroblastoma cell lines. Values are presented as mean $\pm \mathrm{SD}$. Data are representative of three independent experiments with triplicates in each experiment.

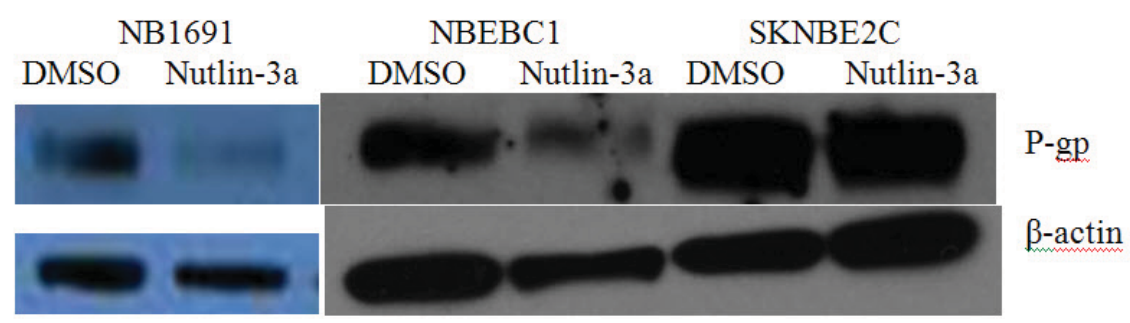

Figure 2-3. P-gp protein expression change after nutlin-3a treatment

Western blot from cell lysates collected after $24-\mathrm{hr}$ nutlin-3a treatment $(20 \mu \mathrm{M})$ probed using antibody c219 against P-gp. $\beta$-actin was used as loading control. Data are representative of one experiment. 
mentioned that this study was preliminary, and more independent experiments are needed to support the change of P-gp expression at the protein level.

As we were planning to conduct further research to study the effect of nutlin-3a on P-gp function, Michaelis et al. in the meantime reported that nutlin-3a inhibited P-gp function and reversed vincristine resistance in neuroblastoma and rhabdomyosarcoma cells independent of p53 status [228]. The mechanism of inhibition proposed in that study was competitive inhibition. In the current study, we evaluated a different mechanism of action of nutlin-3a on P-gp. Instead of competitive inhibition, nutlin-3a inhibited the expression of P-gp. 


\section{CHAPTER 3. MDM2 ANTAGONIST NUTLIN-3A REVERSES MITOXANTRONE RESISTANCE BY INHIBITING BREAST CANCER RESISTANCE PROTEIN MEDIATED DRUG TRANSPORT*}

\subsection{Introduction}

Pre-clinical investigations of the utility of nutlin-3 treatment of cancer cells have focused primarily on the consequences of p53 reactivation in cells due to disruption of the MDM2/p53 interaction. Nutlin-3 is a racemic mixture of nutlin-3a (active enantiomer) and nutlin-3b (inactive enantiomer) with nutlin-3a having 150-fold more affinity to MDM2 [17]. Indeed, single agent nutlin-3 treatment has shown anti-cancer efficacy in xenograft models of solid tumors, including osteosarcoma, prostate cancer, KSHV lymphomas, retinoblastoma, and neuroblastoma [17, 24, 25, 40, 43]. Recently, other effects of nutlin-3 treatment have been reported, including anti-angiogenic effects [214, 229, 230] and radiosensitization of cancer cells under low oxygen conditions [29]. Furthermore, nutlin-3 has been reported to sensitize cancer cells to co-treatment with selected anti-cancer drugs, independent of p53 status, by enhancing the ability of anticancer drugs to activate apoptosis [223], and also by reversing P-gp mediated MDR [228]. Understanding the mechanism behind this nutlin-3 sensitization of resistant cancer cells would significantly enhance the use of nutlin-3 in combination with other anti-cancer drugs in a broad range of tumor types.

Drug-resistance is a major obstacle in the treatment of cancer, and ATP-binding cassette $(\mathrm{ABC})$ transporters play an integral role in the development of multi-drug resistance [231]. ABC transporters utilize the energy of ATP hydrolysis to pump anti-cancer agents out of the cell, thus reducing the intracellular drug concentration. Recently, Michaelis et al. observed that nutlin-3 can interfere with the function of the ABC transporters P-pg and MRP1 [228]. Nutlin-3 treatment reversed drug resistance in neuroblastoma and rhabdomyosarcoma cells over-expressing these transporters in vitro when combined with cytotoxic drugs that are P-gp and MRP1 substrates. These data suggest that nutlin-3 functionally inhibits the action of drug efflux proteins, thereby sensitizing cells to treatment with cytotoxic agents that are substrates of these efflux proteins.

BCRP belongs to the $\mathrm{ABC}$ transporter family. Although it is possible that nutlin-3a may modulate the activity of BCRP, so far, the effect of nutlin-3a on BCRP has not been reported. The present study investigates whether nutlin-3a inhibits BCRP, thus sensitizing cells to enhanced killing by anti-cancer drugs that are BCRP substrates. Using MTS assays, we determined that nutlin-3a reverses resistance to the BCRP substrate mitoxantrone. Combination index calculations indicated synergism when nutlin-3a was

* Reprinted with permission. Zhang, F., et al., MDM2 antagonist nutlin-3a reverses mitoxantrone resistance by inhibiting breast cancer resistance protein mediated drug transport. Biochemical Pharmacology, 2011. 82(1): p. 24-34. 
used in combination with the anticancer agent mitoxantrone, a BCRP substrate, in osteosarcoma cells over-expressing BCRP. Based on these observations, we performed a series of studies to comprehensively investigate the effect of nutlin-3a treatment on BCRP expression and function. Our studies strongly suggest that nutlin-3a inhibits BCRP efflux and can reverse BCRP-related drug resistance, but is not a BCRP substrate.

\subsection{Materials and Methods}

\subsubsection{Reagents}

Nutlin-3a and nutlin-3b were synthesized in the Department of Chemical Biology at St. Jude Children's Research Hospital, Memphis, TN and were solubilized in dimethyl sulfoxide (DMSO) (ATCC, Manassas, VA) to a final concentration of $30 \mathrm{mM}$. The chemical structure of nutlin-3 has been published previously [17]. Hoechst 33342 and G-418 (Geneticin ${ }^{\circledR}$ ) were purchased from Invitrogen (Carlsbad, CA). Mitoxantrone and Ko143 were purchased from Sigma-Aldrich (St. Louis, MO). Fumitremorgin C (FTC) was purchased from Alexis Biochemicals (San Diego, CA).

\subsubsection{Cell culture}

Saos-2 (human osteosarcoma) cells stably transfected with human wild-type (Arg482) BCRP or control vector pcDNA3.1 were generously donated by Dr. John Schuetz (St. Jude Children's Research Hospital, Memphis, TN) [62, 232]. MDCK II- pCDNA3.1 and MDCK II-BCRP cells were generously donated by Dr. Mark Leggas (University of Kentucky, Lexington, KY). Cells were cultured in complete Dulbecco's Modified Eagle's Medium (DMEM) (Invitrogen, Carlsbad, CA) containing 10\% fetal bovine serum (FBS) (Invitrogen, Carlsbad, CA), 1\% L-glutamine (Invitrogen, Carlsbad, CA), and maintained with G418 (0.5 mg / ml) (Invitrogen, Carlsbad, CA). Cells were cultured in G418-free complete media at the time of seeding for individual experiments. Hank's balance salt solution (HBSS) and HEPES buffer were purchased from Invitrogen (Carlsbad, CA).

\subsubsection{Cell viability assay (MTS)}

Cells were seeded in $100 \mu \mathrm{l}$ phenol red-free medium (Invitrogen, Carlsbad, CA) in 96-well plates and allowed to attach overnight. Cells were treated with increasing concentrations of nutlin-3a alone or increasing concentrations of mitroxantrone in combination with $0 \mu \mathrm{M}, 20 \mu \mathrm{M}$ or $50 \mu \mathrm{M}$ nutlin-3a for 24 hours. Cell viabilities were tested by the CellTiter 96® AQueous MTS assay (Promega, Madison, WI) following the manufacturer's instructions. IC $_{50}$ values were calculated using ADAPT 5 (Biomedical Simulations Resources, Los Angeles, CA) [225]. 


\subsubsection{Median effect analysis}

To characterize the interaction between mitroxantrone and nutlin-3a in Saos-2-BCRP and Saos-2-pcDNA3.1 cells, data were analyzed using the median effect method developed by Chou [226]. The combination index (CI) values at non-fixed nutlin-3a/mitoxantrone concentration ratios were calculated using the commercially available software Calcusyn 2.1 (Biosoft, Cambridge, United Kingdom). CI values $<1.0$ indicate synergism, $\mathrm{CI}$ values $=1.0$ indicate additive effect and CI values $>1.0$ indicate antagonism [226].

\subsubsection{Intracellular accumulation and efflux of mitoxantrone by confocal imaging}

Vector control and BCRP expressing Saos-2 cells were seeded on $35 \mathrm{~mm}$ glass bottom dishes (MatTek Corporation, Ashland, MA) and allowed to attach for 36 hours. Cells were pre-incubated with nutlin-3a for 15 minutes in DMEM+ (DMEM with 2\% FBS, $1 \mathrm{mM}$ HEPES buffer) at $37^{\circ} \mathrm{C}$. Mitoxantrone $(1 \mu \mathrm{M})$ was added and cultures were incubated for an additional 1 hour. Cells were washed with ice cold HBSS + (HBSS with 2\% FBS, $1 \mathrm{mM}$ HEPES buffer) containing nutlin-3a and intracellular accumulation was measured using confocal imaging. An Eclipse C1si confocal, configured on an Eclispe TE2000 microscope (Nikon, Melville, NY) with a Plan Fluor $40 \times$ NA 1.3 lens was used. Excitation was from a $642 \mathrm{~nm}$ diode laser, and the emission was collected through a $675 / 50 \mathrm{~nm}$ bandpass filter.

\subsubsection{Hoechst 33342 dye accumulation and efflux studies by flow cytometry}

Hoechst 33342 dye was used as a BCRP substrate. Vector control and BCRP over-expressing Saos-2 and MDCKII cells were cultured to $60-70 \%$ confluence. Single cell suspensions were pre-incubated in DMEM + with nutlin-3a, nutlin-3b or FTC at varying concentrations for 15 minutes at $37^{\circ} \mathrm{C}$. Hoechst 33342 dye was then added to a final concentration of $5 \mu \mathrm{g} / \mathrm{ml}$ and cells were incubated at $37^{\circ} \mathrm{C}$ for 1 hour. Cells were pelleted and resuspended in ice cold HBSS+ containing nutlin-3a, nutlin-3b or FTC. Intracellular Hoechst 33342 fluorescence signals were detected by a 440/40 nm band pass filter with UV laser excitation and the data were collected and analyzed using a BD LSRII flow cytometer (BD, San Jose, CA). Data were processed as previously described [62]. Propidium iodide (PI) (Roche Applied Science, Mannheim, Germany) was added as a marker to label the non-viable cells. Only events from viable cells were used for data analysis.

\subsubsection{Intracellular accumulation and efflux of Hoechst 33342 by widefield imaging}

Vector control and BCRP expressing Saos-2 cells were seeded on $35 \mathrm{~mm}$ glass bottom dishes and allowed to attach for 36 hours. Cells were pre-incubated with nutlin-3a 
or nutlin-3b in DMEM+ for 15 minutes at $37^{\circ} \mathrm{C}$. Hoechst $33342(1 \mu \mathrm{g} / \mathrm{ml})$ was added and cultures were incubated for an additional 1 hour. Cells were washed with ice cold HBSS + containing nutlin-3a or nutlin-3b and intracellular accumulation was measured using widefield fluorescence imaging. Hoechst 33342 imaging was performed on a Nikon Eclipse TE2000 microscope with a Plan Fluor $40 \times$ NA 0.6 lens and a standard DAPI filter set.

\subsubsection{Western blots}

Total cellular protein was extracted from cell pellets and protein concentrations were determined by BCA assay (Thermo Scientific, Rockford, IL). Proteins were resolved by SDS PAGE [4-12\% gradient Bis/Tris NuPage gels (Invitrogen, Carlsbad, CA)] with MOPS SDS running buffer (Invitrogen, Carlsbad, CA) before transferring onto InvitrolonTM PVDF membranes (Invitrogen, Carlsbad, CA). BCRP was detected using the rat monoclonal BXP-53 (Alexis Biochemicals, San Diego, CA). P-gp was detected using the mouse monoclonal antibody clone C-219 (Alexis Biochemicals, San Diego, CA). Beta-actin (AC-15, Sigma-Aldrich, St. Louis, MO) was used as the loading control.

\subsubsection{Flow cytometry for BCRP}

PE conjugated mouse anti-BCRP (MAB4155P) (Millipore, Billerica, MA) antibody was used to detect protein expression by flow cytometry. Cells were treated

with $50 \mu \mathrm{M}$ nutlin-3a for 1 hour, harvested, and then $1.0 \times 10^{6}$ cells were resuspended in $100 \mu \mathrm{L}$ BD Fc BlockTM (BD Pharmingen, San Diego, CA) and incubated on ice for 30 minutes. Cells were washed once and then stained with the primary antibody (anti-BCRP $10 \mu \mathrm{g} / \mathrm{ml}$ ) at room temperature for 30 minutes. Cells were again washed and resuspended in a final volume of $0.5 \mathrm{ml}$. Cells were counterstained with 4'-6-Diamidino-2-phenylindole (DAPI) (Invitrogen, Carlsbad, CA) and analyzed using a BD LSRII flow cytometer (BD, San Jose, CA).

\subsubsection{BCRP localization}

Localization of BCRP was evaluated using confocal imaging analysis. Cells were grown on $35 \mathrm{~mm}$ glass bottom dishes and treated with $50 \mu \mathrm{M}$ nutlin-3a for 90 minutes. After incubation, cells were washed with HBSS and fixed with 4\% paraformaldehyde (Polysciences, Warrington, PA) for 15 minutes at room temperature. After 3 HBSS washes, cells were permeabilized using $0.2 \%$ Triton X-100 (Sigma-Aldrich, St. Louis, MO) diluted in HBSS for 5 minutes then washed in HBSS. After 3 HBSS washes, Image-iT ${ }^{\mathrm{TM}} \mathrm{FX}$ signal enhancer solution (Invitrogen, Carlsbad, CA) was applied and the dishes were incubated at room temperature for 30 minutes. After 3 additional HBSS washes, mouse monoclonal antibody against BCRP (BXP-21, Alexis Biochemicals, San Diego, CA) (1:1000 diluted in HBSS $+2 \%$ bovine serum albumin) was added and cells were incubated for an additional 60 minutes at room temperature. Cells were then stained 
with Alexa Fluor ${ }^{\circledR} 555$ goat anti-mouse IgG secondary detection conjugate (1:500) (Invitrogen, Carlsbad, CA) at room temperature for 90 minutes. Finally, ProLong ${ }^{\circledR}$ Gold-DAPI anti-fade reagent (Invitrogen, Carlsbad, CA) was added and wells were cover-slipped. BCRP localization was assessed using confocal imaging. A Nikon Eclipse C1si confocal configured on an Eclipse TE2000 microscope with a Plan Fluor $40 \times$ NA 1.3 lens was used. Excitation was from a $561 \mathrm{~nm}$ diode laser and emission was collected through a $605 / 75 \mathrm{~nm}$ bandpass filter.

\subsubsection{Intracellular accumulation of nutlin-3a}

MDCKII-pcDNA3.1 and MDCKII-BCRP cells were seeded into 6-well plates at a density of $0.5 \times 10^{5}$ cells/well in $2 \mathrm{ml}$ of complete DMEM without G418. When cells were $80 \sim 90 \%$ confluent, nutlin-3a was added at increasing final concentrations of $0,1,5$, 10,20 , and $50 \mu \mathrm{M}$. Cells were incubated at $37^{\circ} \mathrm{C}$ for 1 hour. Cells were then washed with ice cold phosphate buffered saline (PBS) twice, and scraped off in $1 \mathrm{ml}$ ice cold homogenization buffer (5 mM HCOONH4, pH 7.0). Cell pellets were then lysed on ice by sonicating for approximately $10 \mathrm{sec} /$ well twice with a $15 \mathrm{sec}$ interval using a 4710 series ultrasonic homogenizer (Cole-Parmer, Chicago, IL, USA). Intracellular nutlin-3a concentrations were measured using the LC-MS/MS method published previously [233]. Final intracellular nutlin-3a concentrations were normalized to total protein content as measured by BCA assay.

\subsubsection{Bi-directional transport across MDCKII monolayer cells}

MDCKII-pCDNA3.1 and MDCKII-BCRP cells were seeded at $1 \times 10^{6}$ cells per well in $0.4 \mu \mathrm{m}, 12 \mathrm{~mm}$ Transwell ${ }^{\circledR}$ permeable inserts (Corning Incorporated, Corning, NY). Transport assays were performed when cells reached consistent trans-epithelial electrical resistance [234] values (between 200-300 $\mu \Omega \mathrm{cm}^{2}$ ), indicating that the cells had formed a confluent polarized monolayer. Prior to transport assays (30 minutes), the medium in the donor and receiver chambers was removed and replaced with transport buffer (HBSS/25 mM HEPES). Buffer containing nutlin-3a was added to either the apical or the basolateral side of the monolayer with or without the BCRP-specific inhibitor Ko143. Samples were removed for the determination of initial nutlin-3a concentrations, and then at 30,60,120, and 240 minutes. $50 \mu 1$ aliquots were removed from the either the basolateral or apical compartments. The volume removed was replaced immediately with fresh transport buffer. Nutlin-3a samples were prepared and analyzed using LC-MS/MS method as described above.

The apparent permeability (Papp) is calculated using Equation 3-1 [234]:

$$
\text { Papp }=\left(\mathrm{Vr} / \mathrm{C}_{0}\right)(1 / \mathrm{S})(\mathrm{dC} / \mathrm{dt})
$$


where $\mathrm{V}_{\mathrm{r}}$ is the volume of buffer in the receiver chamber; $\mathrm{C}_{0}$ is the initial drug concentration in the donor chamber; $\mathrm{S}$ is the surface area of monolayer; $\mathrm{dC} / \mathrm{dt}$ is the linear slope of drug concentration in the receptor chamber over time.

The efflux ratio (RE) is calculated using Equation 3-2:

$$
\mathrm{RE}=\left(\mathrm{P}_{\mathrm{app}, \mathrm{B}-\mathrm{A}} / \mathrm{P}_{\mathrm{app}, \mathrm{A}-\mathrm{B}}\right)
$$

where $\mathrm{P}_{\mathrm{app}, \mathrm{B}-\mathrm{A}}$ is the apparent permeability of drug transport from basolateral to apical side and $\mathrm{P}_{\text {app,A-B }}$ is the apparent permeability of drug transport from apical to basolateral side.

The final efflux ratio (R) is calculated using Equation 3-3:

$\mathrm{R}=($ MDCK II-BCRP efflux ratio)/(MDCK II-pcDNA 3.1 efflux ratio) $\quad$ Eq. 3-3

\subsubsection{ATPase assay}

BCRP ATPase activity was determined using SB BCRP HAM PREDEASYTM ATPase kit and SB defBCRP HAM PREDEASYTM Ctrl kit following the manufacturer's instructions (XenoTech, Lenexa, KS). The assay contains two different tests that are performed simultaneously on the same plate. In the activation test, BCRP substrates stimulate baseline vanadate-sensitive ATPase activity. In the inhibition test, inhibitors or slowly transported compounds may inhibit the maximal vanadate sensitive ATPase activity. Nutlin-3a was tested in both the activation and inhibition reactions at increasing concentrations $(0.14,0.41,1.23,3.70,11.11,33.33,100$, and $150 \mu \mathrm{mol} / \mathrm{L})$ for 10 minutes following the manufacturer's instructions. The assay was performed in triplicate.

\subsubsection{Statistical analysis}

All data expressed as mean \pm standard deviation unless otherwise indicated. Data were analyzed for statistical significance using Student's t test. Differences with $p<0.05$ were considered statistically significant. Linear and nonlinear regressions were performed using Prism 5 (GraphPad Software, La Jolla, CA).

\subsection{Results}

\subsubsection{Nutlin-3a sensitizes BCRP expressing cells to mitoxantrone treatment}

Saos-2-pcDNA3.1 and Saos-2-BCRP cells were incubated with nutlin-3a alone or in combination with the anti-cancer agent mitoxantrone, a BCRP substrate. $\mathrm{IC}_{50}$ values were determined by MTS assay. BCRP over-expression did not confer resistance to 
nutlin-3a as a single agent. The $\mathrm{IC}_{50}$ value of nutlin-3a was $45.8( \pm 2.6) \mu \mathrm{M}$ for Saos-2-BCRP and $43.5( \pm 3.0) \mu \mathrm{M}$ for Saos-2-pcDNA3.1 $(\mathrm{p}>0.05)$ (Figure 3-1).

BCRP expression did confer resistance to mitoxantrone in the Saos-2 cell lines. The Saos-2-pcDNA3.1 cells exhibited sensitivity to mitoxantrone with an $\mathrm{IC}_{50}$ of 2.0 $( \pm 0.1) \mu \mathrm{M}$ while the BCRP over-expressing Saos-2 cells had a markedly increased mitoxantrone $\mathrm{IC}_{50}$ of $165.8( \pm 21.9) \mu \mathrm{M}(\mathrm{P}<0.001)$ (Figure 3-2). To determine the effect of nutlin-3a on potentially reversing this mitoxantrone resistance, Saos-2-BCRP cells were co-incubated with $20 \mu \mathrm{M}$ or $50 \mu \mathrm{M}$ nutlin-3a and increasing concentrations of mitoxantrone $(0.01-300 \mu \mathrm{M})$ for 24 hours. A dramatic reduction in the mitoxantrone $\mathrm{IC}_{50}$ from $165.8( \pm 21.9) \mu \mathrm{M}$ to $7.6( \pm 0.5) \mu \mathrm{M}(21.8$-fold, $\mathrm{p}<0.001)$ and $1.0( \pm 0.07) \mu \mathrm{M}$ (165.8-fold, $\mathrm{p}<0.001)$ was observed in the Saos-2-BCRP cells after treatment with 20 and $50 \mu \mathrm{M}$ nutlin-3a, respectively (Figure 3-2). In Saos-2-pcDNA3.1 cells, only a moderate reduction of mitoxantrone $\mathrm{IC}_{50}$ was observed, from $2.0( \pm 0.01) \mu \mathrm{M}$ to 1.4 $( \pm 0.1) \mu \mathrm{M}(1.4$-fold, $\mathrm{p}<0.001)$ and $0.6( \pm 0.4) \mu \mathrm{M}(3.3$-fold, $\mathrm{p}<0.001)$ (Figure 3-2). Using combination index analysis, we evaluated whether the combination of nutlin-3a and mitoxantrone was synergistic. Combination index $(\mathrm{CI})$ values around 1 indicate that two drugs have an additive effect. A CI $<1$ indicates synergy, and a CI $>1$ indicates antagonism [226]. The fractional effect is the ratio of the effect (growth inhibition) caused by the two compounds in combination to that of one of the compounds alone. A fractional effect value of 0 indicates no inhibition and fractional effect value of 1 indicates $100 \%$ inhibition of cell viability. In contrast to Saos-2-pcDNA3.1 cells where synergism, additivity, and antagonism can be observed on different nutlin-3a:

mitoxantrone ratios (Figure 3-3A), moderate $(++)$ to strong $(++++)$ synergism [226] was observed in Saos-2-BCRP cells with combination index (CI) values between 0.132 and 0.798 at all nutlin-3a: mitoxantrone ratios tested (Figure 3-3B). These results indicate that the synergistic effect observed in Saos-2-BCRP cells induced by the nutlin-3a/mitoxantrone combination is dependent on the presence of BCRP.

\subsubsection{Nutlin-3a inhibits BCRP-mediated transport of mitoxantrone}

To determine whether the observed reduction in the mitoxantrone $\mathrm{IC}_{50}$ of the Saos-2-BCRP cells co-treated with nutlin-3a was due to increased exposure to mitoxantrone, intracellular accumulation of mitoxantrone was measured by confocal imaging. Saos-2-pcDNA3.1 and Saos-2-BCRP cells were co-incubated with $1 \mu \mathrm{M}$ mitoxantrone and increasing concentrations of nutlin-3a ( 0 to $50 \mu \mathrm{M})$ for 1 hour. In the absence of nutlin-3a, little mitoxantrone accumulation was observed in Saos-2-BCRP cells (Figure 3-4), indicating active efflux by BCRP, whereas under the same conditions, mitoxantrone was retained in the Saos-2-pcDNA3.1 cells. Treatment with nutlin-3a resulted in a dose-dependent increase in the intracellular mitoxantrone accumulation in the Saos-2-BCRP cells, suggesting a decrease in BCRP efflux function. 


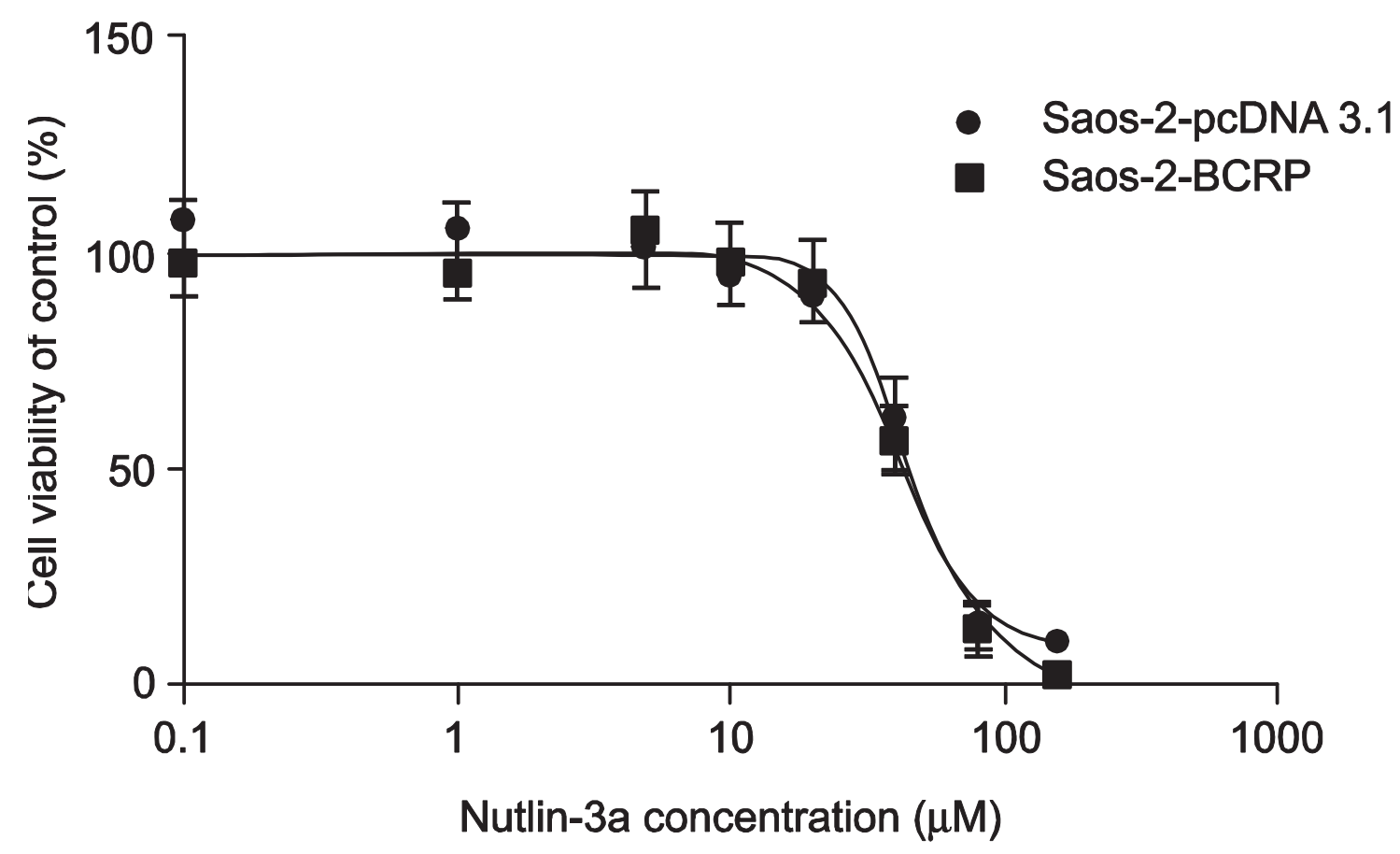

Figure 3-1. BCRP expression does not confer resistance to nutlin-3a treatment

Saos-2-BCRP and Saos-2-pcDNA3.1 cells were incubated with increasing concentrations of nutlin-3a $(0.1-150 \mu \mathrm{M})$ for 24 hours. Cell viabilities were tested by MTS assay in triplicate. $\mathrm{IC}_{50}$ values were calculated using ADAPT 5. Values are presented as mean \pm SD. Data are representative of three independent experiments. 


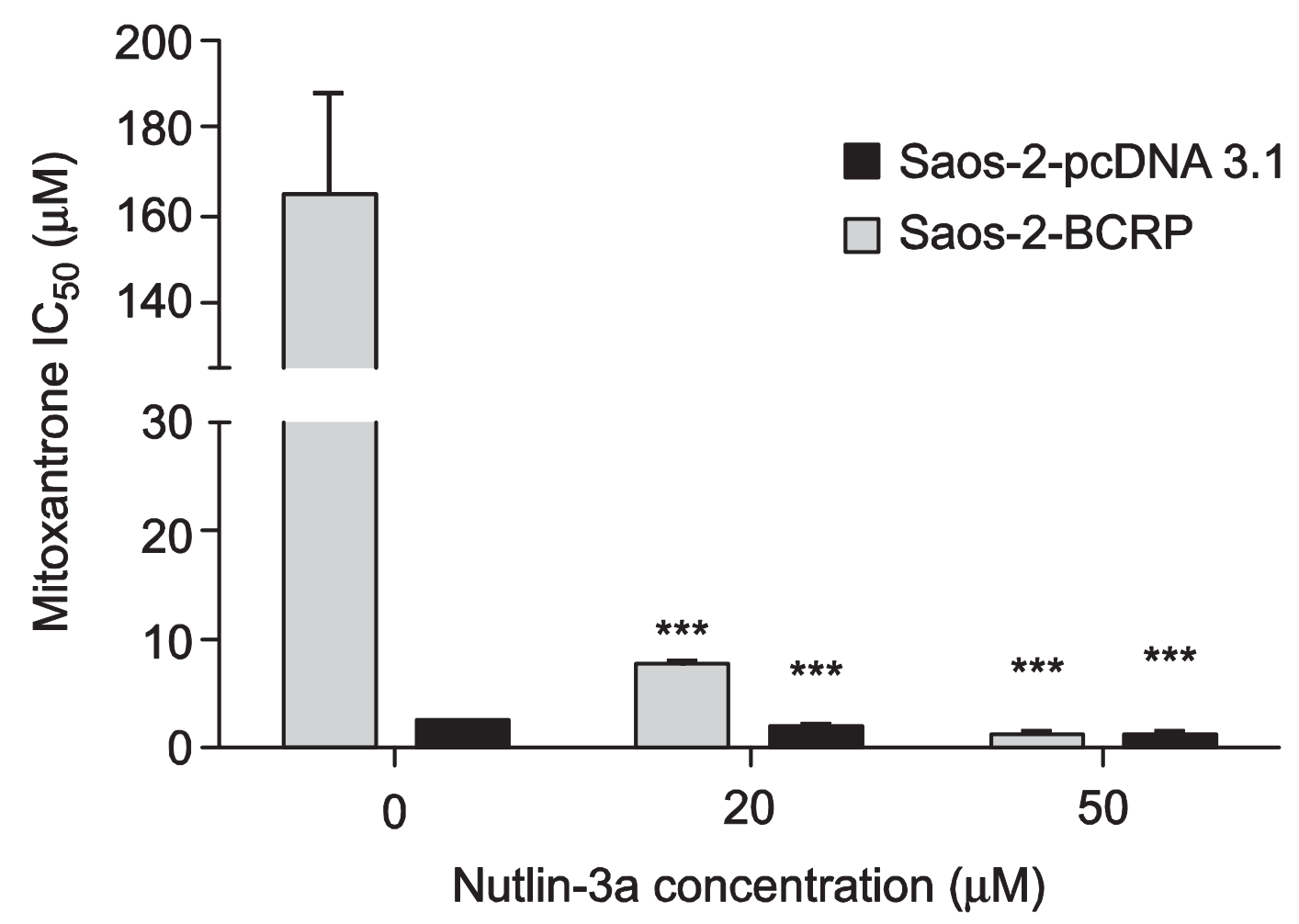

Figure 3-2. Co-treatment of cells with nutlin-3a and mitoxantrone strongly reverses BCRP mediated drug resistance to mitoxantrone

Saos-2-pcDNA3.1 and Saos-2-BCRP cells were treated with increasing concentrations of mitoxantrone $(0.01-300 \mu \mathrm{M})$ in combination with 20 and $50 \mu \mathrm{M}$ nutlin-3a for 24 hours as described in the methods. Cell viabilities were determined by MTS assay in triplicate.

ADAPT 5 was used to calculate $\mathrm{IC}_{50}$. Values are presented as mean $\pm \mathrm{SD}$. $* * * \mathrm{P}<0.001$. Data are representative of two independent experiments. 
A. Saos-2-pcDNA 3.1
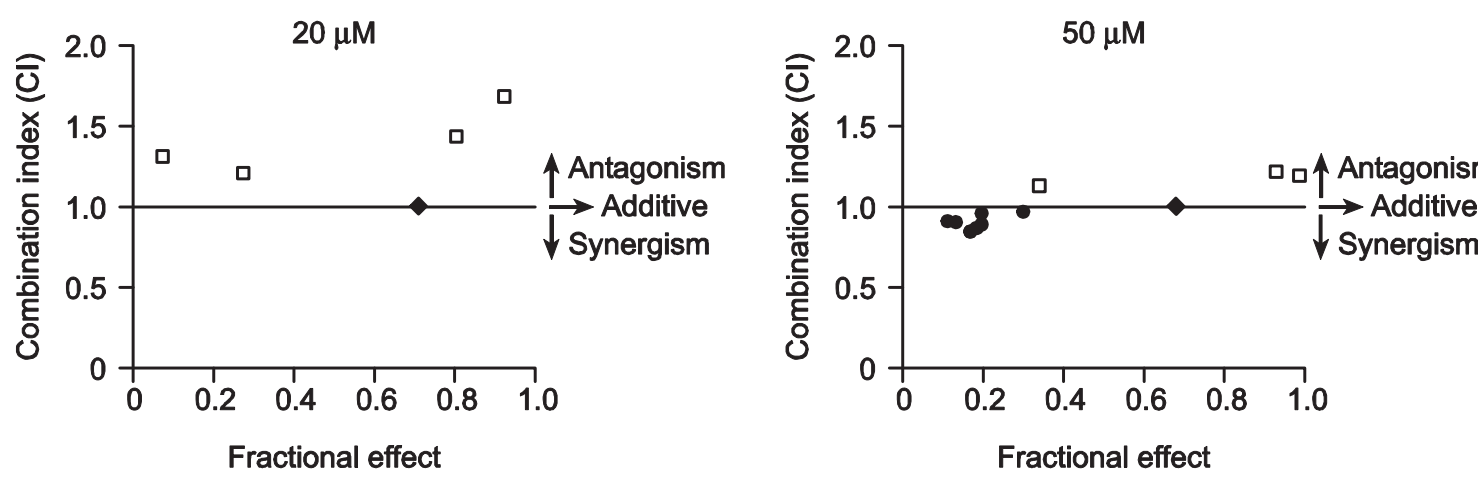

B. Saos-2-BCRP
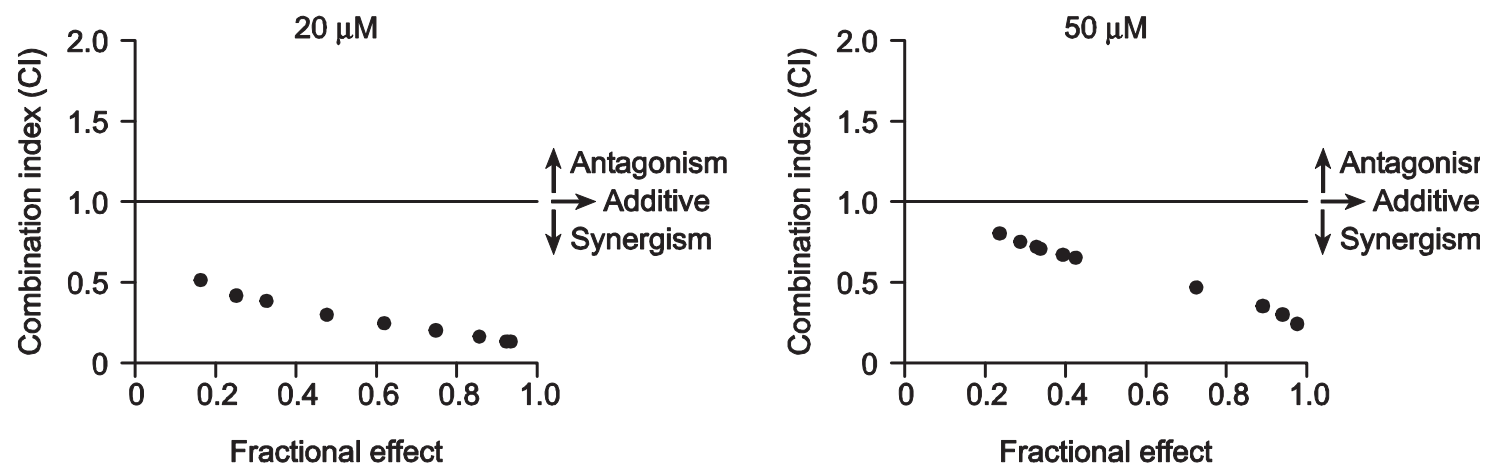

Figure 3-3. Synergistic effects of nutlin-3a in combination with mitoxantrone

The combination index (CI) values at non-fixed nutlin-3a/mitoxantrone concentration ratios were calculated using commercially available software Calcusyn 2.1 • CI values $<1.0$ indicate synergism, $\diamond \mathrm{CI}$ values $=1.0$ indicate additive effect, and $\square \mathrm{CI}$ values $>1.0$ indicate antagonism. Fractional effect is defined as the fraction of cells affected by nutlin-3a and mitoxantrone combination. A fractional effect value of 0 indicates no inhibition and a fractional effect value of 1 indicates $100 \%$ inhibition of cell viability. Data are representative of two independent experiments.

A. For Saos-2-pcDNA3.1 cells, at $20 \mu \mathrm{M}$ nutlin-3a, additive effect and antagonism were observed. At $50 \mu \mathrm{M}$ nutlin-3a, when nutlin-3a: mitoxantrone ratios were $>100: 1$, both additive and synergistic effects were observed; when nutlin-3a: mitoxantrone ratios were $<100$ :1, antagonism was observed.

B. For Saos-2-BCRP cells, moderate $(++)$ to strong $(++++)$ synergism was observed at all ratios tested. 


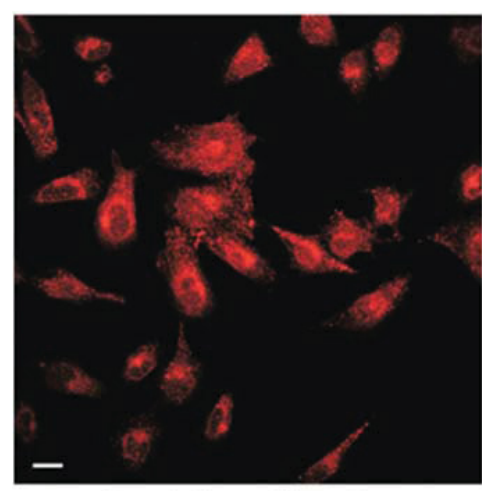

$0 \mu \mathrm{M}$ nutlin-3a

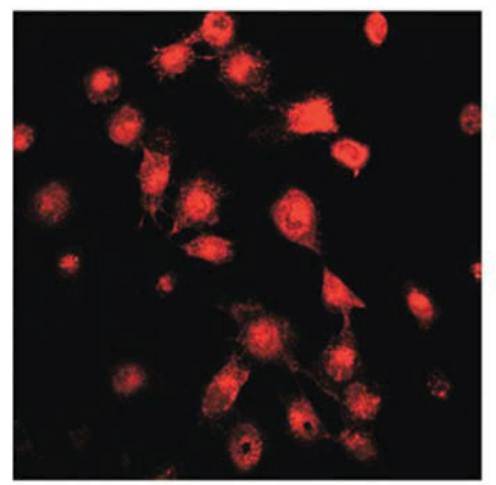

$50 \mu \mathrm{M}$ nutlin-3a

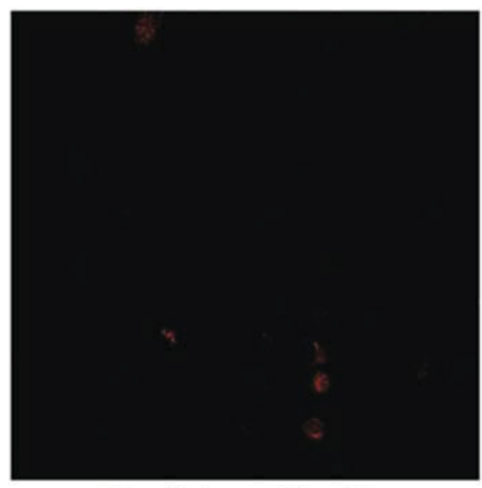

$0 \mu \mathrm{M}$ nutlin-3a

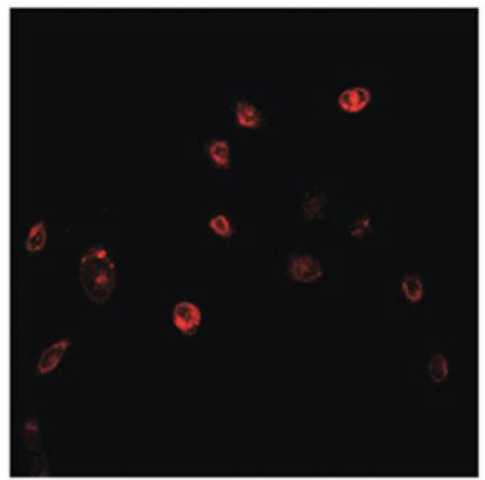

$20 \mu \mathrm{M}$ nutlin-3a

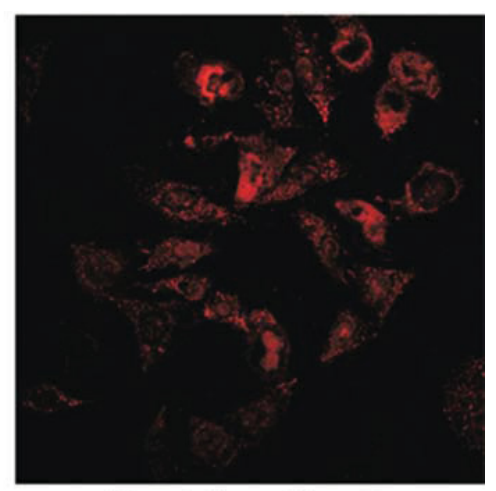

$10 \mu \mathrm{M}$ nutlin-3a

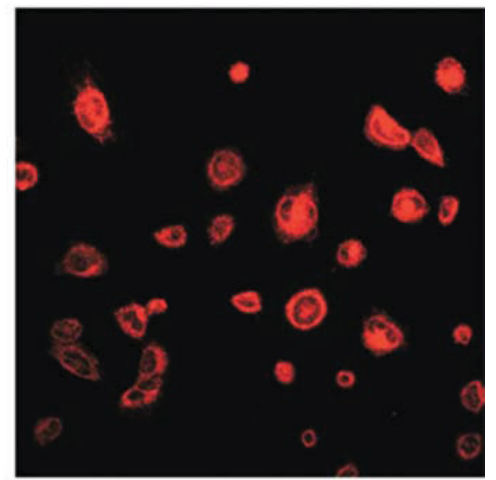

$50 \mu \mathrm{M}$ nutlin-3a

Saos-2-pcDNA 3.1

Figure 3-4. Nutlin-3 treatment strongly increases the intracellular accumulation of mitoxantrone in Saos-2-BCRP cell lines

Nutlin-3 treatment strongly increases the intracellular accumulation of mitoxantrone in Saos-2-BCRP cell lines. Confocal imaging of mitoxantrone (red) in Saos-2-pcDNA3.1 and Saos-2-BCRP cells in the presence increasing concentrations of nutlin-3a for 60 minutes suggested dose dependent restoration of intracellular mitoxantrone accumulation. Scale bar, $30 \mu \mathrm{m}$. Data are representative of three independent experiments. 


\subsubsection{Nutlin-3a inhibits BCRP-mediated transport of Hoechst 33342}

To determine whether nutlin-3a could also reduce the efflux of other BCRP substrates, the intracellular retention of another prototypical BCRP substrate, Hoechst 33342 , was measured by flow cytometry in Saos-2 cells with and without BCRP expression. As BCRP over-expressing Saos-2 cells were incubated with increasing amounts of nutlin-3a, a dose-dependent decrease in the efflux of Hoechst 33342 was observed. Co-incubation of cells with nutlin-3a and Hoechst 33342 for 1 hour resulted in an almost complete inhibition of Hoechst 33342 efflux in Saos-2-BCRP cells (Figure 3-5A). This inhibition was comparable to that seen with the BCRP-specific inhibitor FTC (Figure 3-6). Co-incubation of cells with enantiomer nutlin-3b resulted in the same reduction in Hoechst 33342 efflux (Figure 3-7). Only events from viable cells were used for data analysis, and there was no difference in the viability between BCRP over-expressing and the vector control cells treated with either nutlin-3a or nutlin-3b (Figure 3-8). To determine if the reduced Hoechst 33342 efflux was dependent on p53 status, accumulation studies were also performed in p53 wild-type MDCKII cells. The results indicated that nutlin-3a also reverses the Hoechst 33342 efflux in a p53 wild-type cell line in a dose dependent manner (Figure 3-5B). Hence, nutlin-3a inhibition of BCRP-mediated transport of Hoechst 33342 is independent of cellular p53 status. In addition to the abrogation of efflux observed with flow cytometric methods, results from fluorescence imaging also demonstrated after treatment with nutlin-3a that Hoechst 33342 intracellular accumulation was dramatically restored in the Saos-2-BCRP cell line to levels comparable to Saos-2-pcDNA3.1 control cells (Figure 3-9). Treatment of cells with the enantiomer nutlin-3b resulted in the same reduction in Hoechst 33342 efflux, demonstrating that the two enantiomers have comparable effects on BCRP function (Figure 3-9).

\subsubsection{Nutlin-3a treatment does not alter BCRP expression or localization}

To determine whether increased accumulation and sensitivity to mitoxantrone were a result of nutlin-3a inducing an alteration in BCRP protein levels, Saos-2-pcDNA3.1 and Saos-2-BCRP cells were treated at the highest nutlin-3a dose level used for functional studies ( $50 \mu \mathrm{M} ; 1 \mathrm{hr})$ and BCRP protein levels were measured. As shown in Figure 3-10, both western blot (Figure 3-10A) and flow cytometric analysis (Figure 3-10B) demonstrated that total cellular protein levels of BCRP in Saos-2-BCRP cells were not altered in the presence of nutlin-3a. Since nutlin-3 has been shown to affect P-gp function, levels of P-gp protein were also assessed by western blot. Although Saos-2 cells do not express detectable levels of P-gp [232, 235], it is possible that nutlin-3a treatment may up-regulate P-gp expression. As expected, nutlin-3a treatment did not significantly alter the expression of P-gp in either Saos-2-pcDNA3.1 or -BCRP cells (data not shown). Additional studies using confocal microscopy confirmed no obvious

alteration in the membrane translocation of BCRP protein (Figure 3-11). 


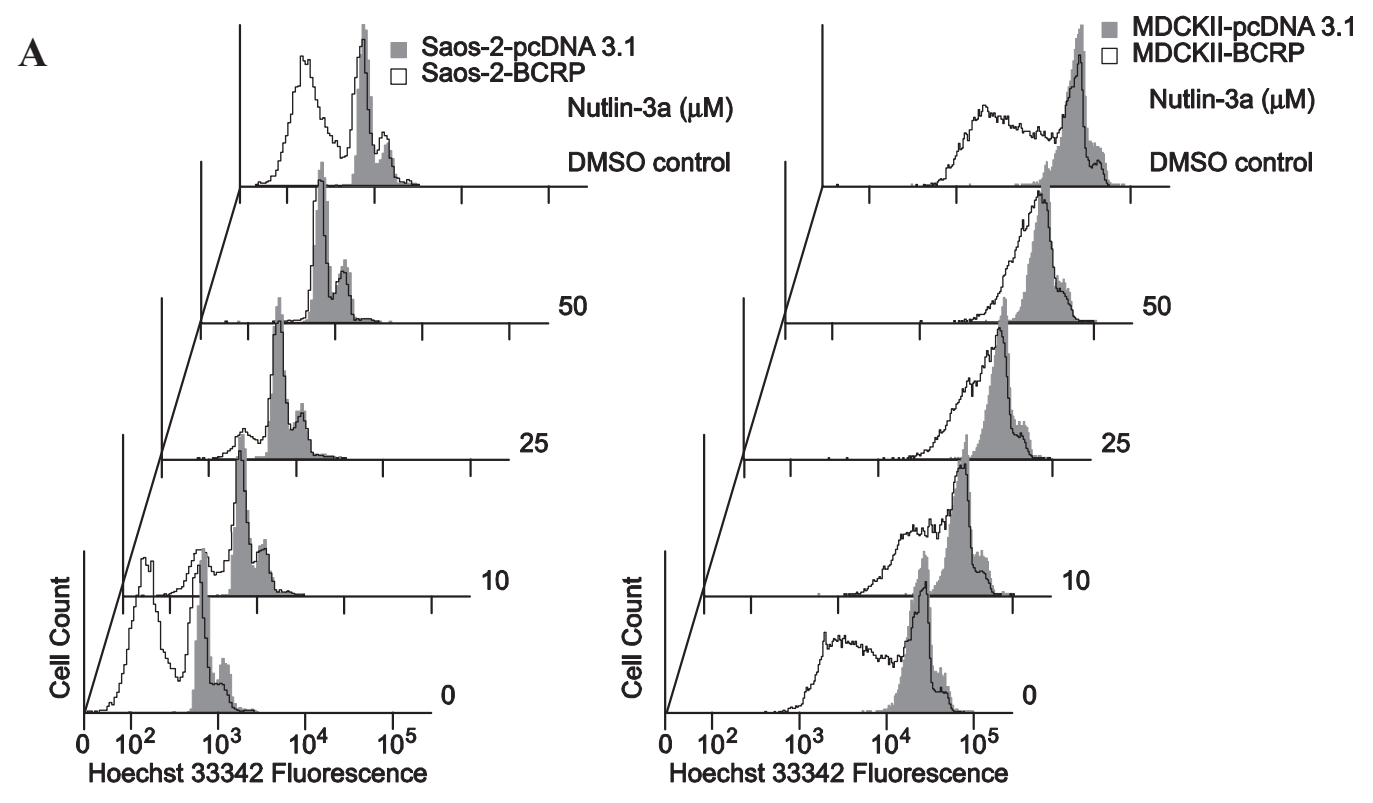

B
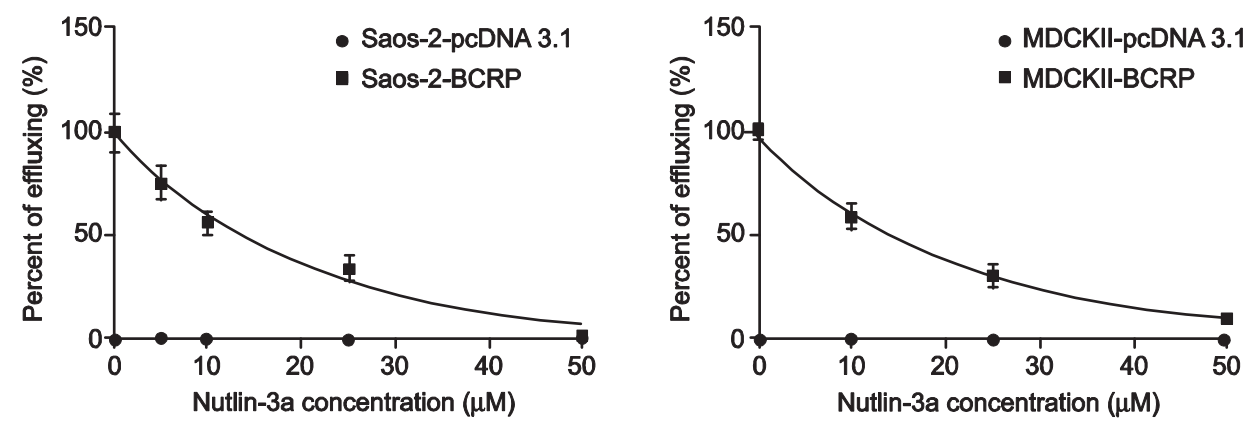

Figure 3-5. Nutlin-3a dose-dependently inhibits BCRP efflux of Hoechst 33342, independent of p53 status

p53 null Saos-2-pcDNA3.1 and Saos-2-BCRP, and p53 wild-type MDCKII-pcDNA3.1 and MDCKII-BCRP cells were incubated with increasing concentrations of nutlin-3a or vehicle control in the presence of Hoechst 33342 for 60 minutes. Data are representative of at least three independent experiments.

A. Dose-dependent reduction of Hoechst 33342 efflux after nutlin-3a treatment assayed by flow cytometry in Saos-2 and MDCKII cells.

B. Quantitative assessment of nutlin-3a effects from representative flow cytometry experiments. Values are presented as mean $\pm \mathrm{SD}$. 


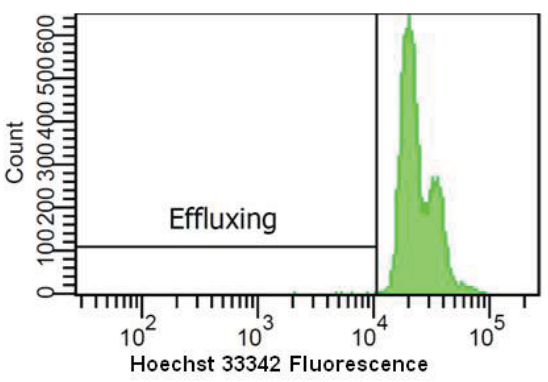

Saos-2-pcDNA3.1 DMSO

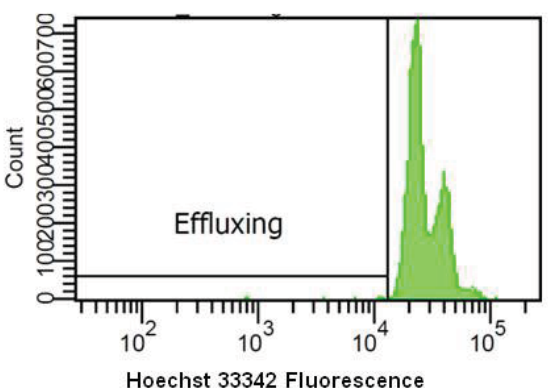

Saos-2-pcDNA3.1 FTC $12 \mu \mathrm{M}$

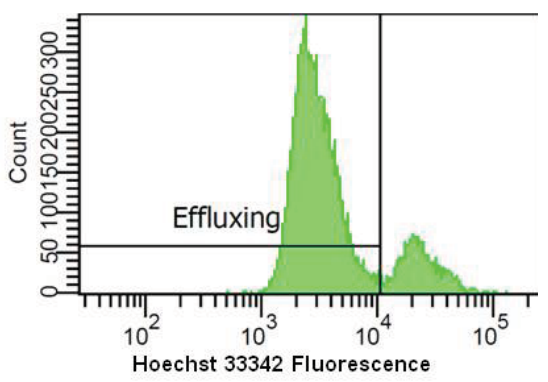

Saos-2-BCRP DMSO

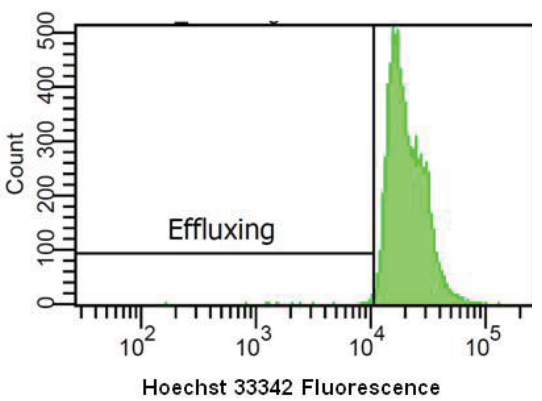

Saos-2-BCRP FTC $3 \mu \mathrm{M}$

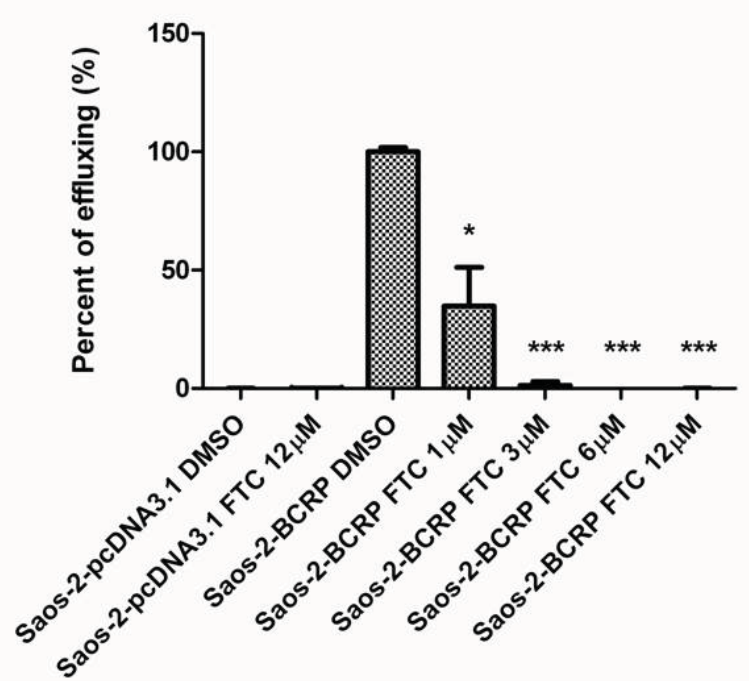

Figure 3-6. Effect of FTC on the BCRP efflux of Hoechst 33342

Saos-2-pcDNA3.1 and Saos-2-BCRP cells were incubated with DMSO or FTC in the presence of Hoechst 33342 for 60 minutes. Data are representative of one experiment performed in triplicate. Error bar, \pm SD. ${ }^{*} p<0.05, * * * p<0.0001$. 


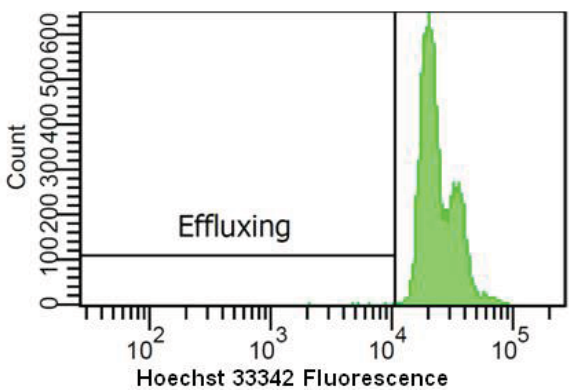

Saos-2-pcDNA3.1 DMSO

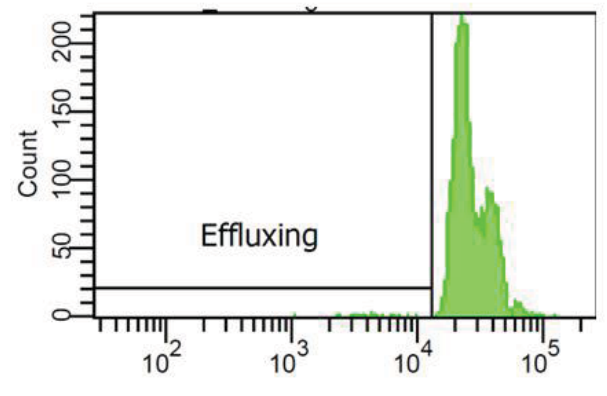

Hoechst 33342 Fluorescence

Saos-2-pcDNA3.1 nutlin-3b $50 \mu \mathrm{M}$

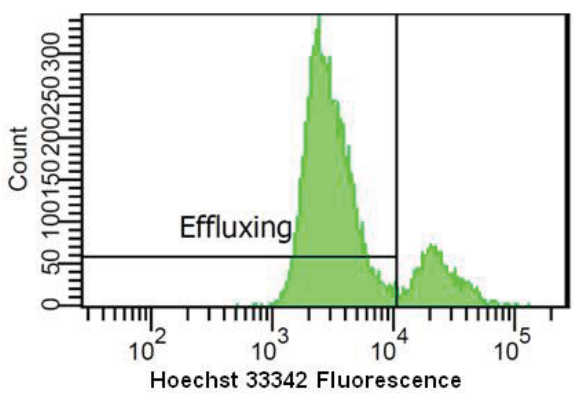

Saos-2-BCRP DMSO

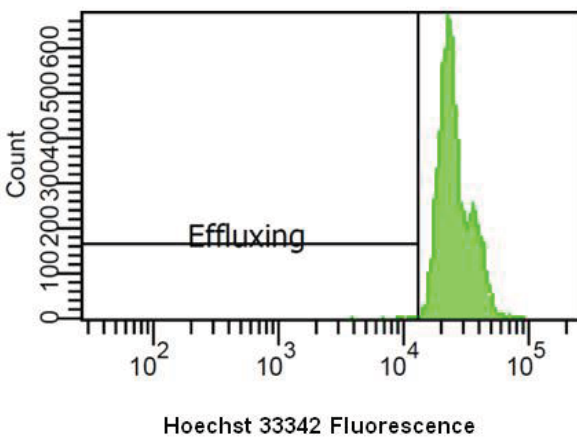

Saos-2-BCRP nutlin-3b $50 \mu \mathrm{M}$

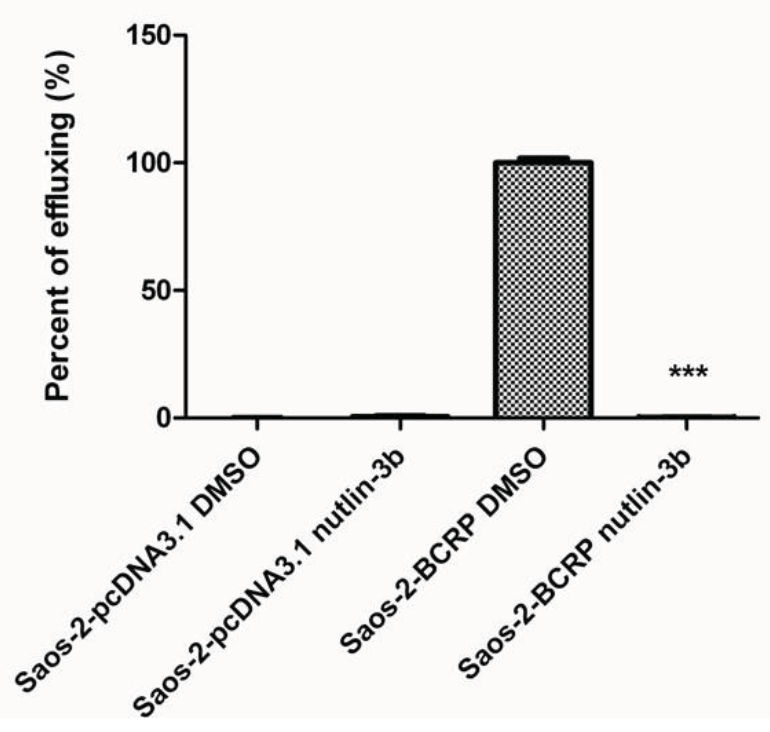

Figure 3-7. Effect of nutlin-3b on efflux of Hoechst 33342

Saos-2-pcDNA3.1 and Saos-2-BCRP cells were incubated with DMSO or nutlin-3b in the presence of Hoechst 33342 for 60 minutes. Data are representative of one experiment performed in triplicate. Error bar, \pm SD. ${ }^{* *} \mathrm{p}<0.0001$. 


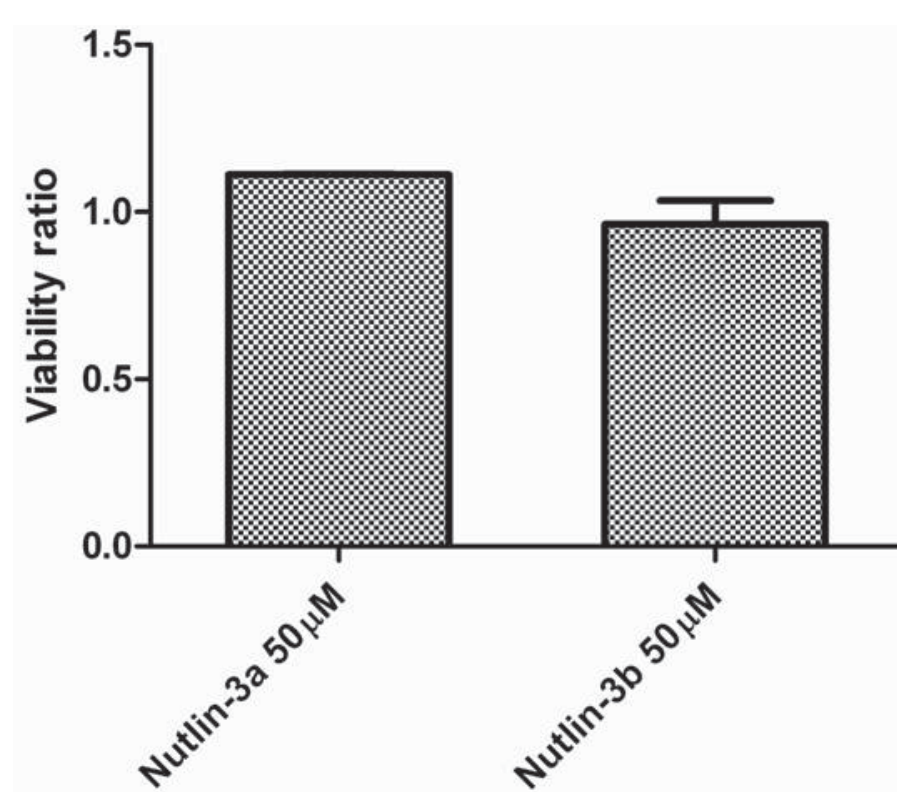

Figure 3-8. Viability ratio of Saos-2-BCRP/ Saos-2-pcDNA3.1 during the Hoechst 33342 efflux study

Saos-2-pcDNA3.1 and Saos-2-BCRP cells were incubated with $50 \mu \mathrm{M}$ nutlin-3a or nutlin-3b in the presence of Hoechst 33342 for 60 minutes. Data are representative of one experiment performed in duplicate. Error bar, \pm SD. $p>0.05$. 
Saos-2-pcDNA 3.1
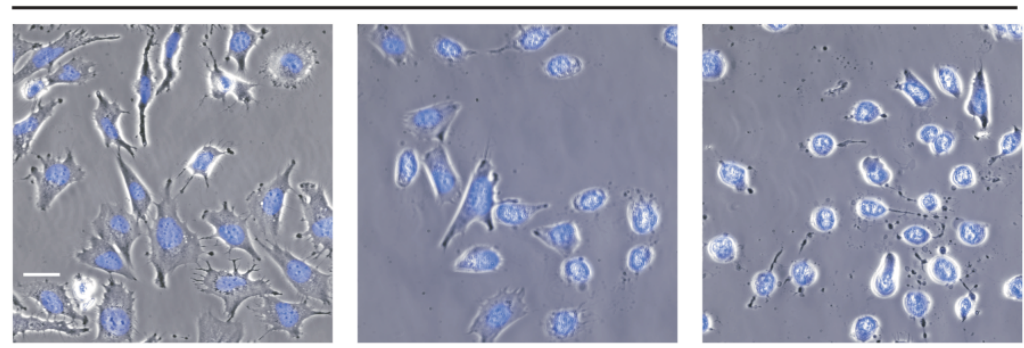

Bright-field

overlay
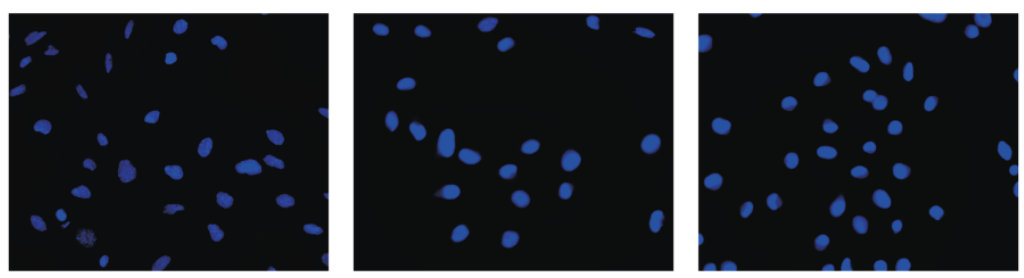

Hoechst 33342

flourescence

$0 \mu \mathrm{M}$ nutlin-3a

$50 \mu \mathrm{M}$ nutlin-3a

$50 \mu \mathrm{M}$ nutlin-3b

\section{Saos-2-BCRP}
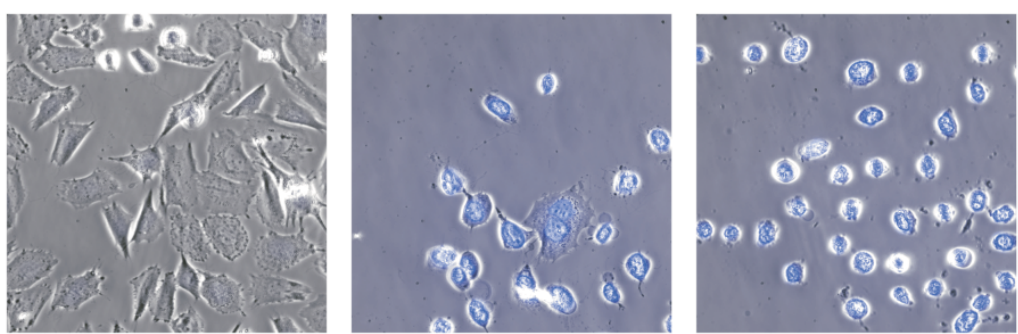

Bright-field

overlay
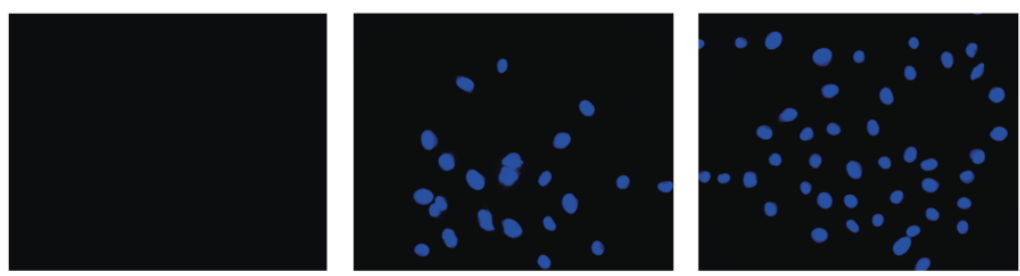

Hoechst 33342

flourescence

$0 \mu \mathrm{M}$ nutlin-3a

$50 \mu \mathrm{M}$ nutlin-3a

$50 \mu \mathrm{M}$ nutlin-3b

Figure 3-9. Nutlin-3 treatment increases the intracellular accumulation of Hoechst 33342 in Saos-2-BCRP cell lines

Wide-field fluorescence imaging of intracellular Hoechst 33342 (blue) in Saos-2-pcDNA3.1 and Saos-2-BCRP cells in the presence or absence of $50 \mu \mathrm{M}$ nutlin-3a or nutlin-3b for 60 minutes. Treatment with nutlin- $3 a$ and nutlin- $3 b$ strongly restored the accumulation of Hoechst 33342 in Saos-2-BCRP cells. Data are representative of three independent experiments. 


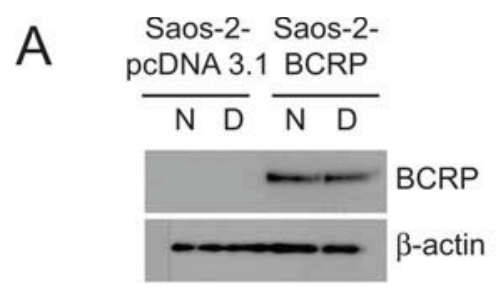

B

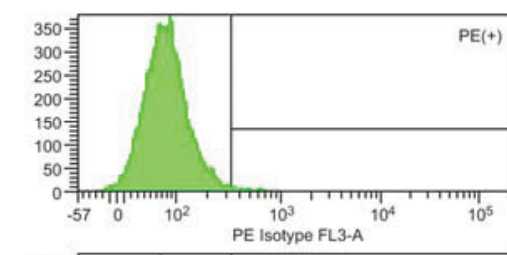

Isotype control
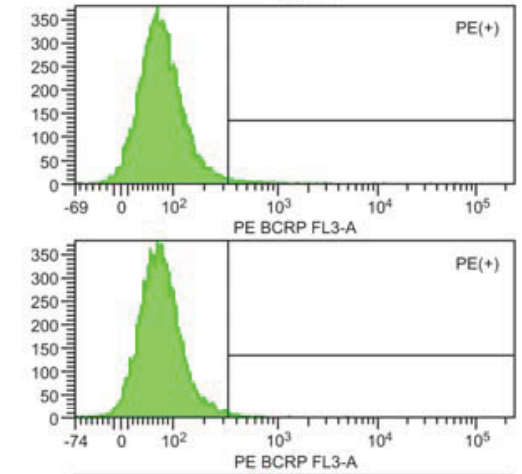

Nutlin-3a

DMSO

ले
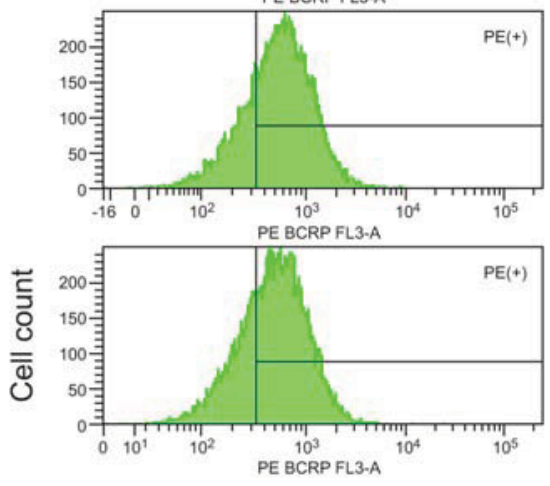

Nutlin-3a

\section{Figure 3-10. Nutlin-3a does not alter levels of BCRP protein expression}

A. Western blot analysis of the BCRP protein expression following nutlin-3a treatment (N, $50 \mu \mathrm{M}$ nutlin-3a; D, equal volume DMSO). BCRP protein was detected using the monoclonal antibody BXP-53.

B. Flow cytometric analysis of the cell-surface expression of BCRP in response to nutlin-3a treatment. Cells were stained with the PE conjugated mouse anti-BCRP antibody (MAB4155P) and subjected to analysis. DAPI was added to cells to indicate viability. Data are representative of two independent experiments. 

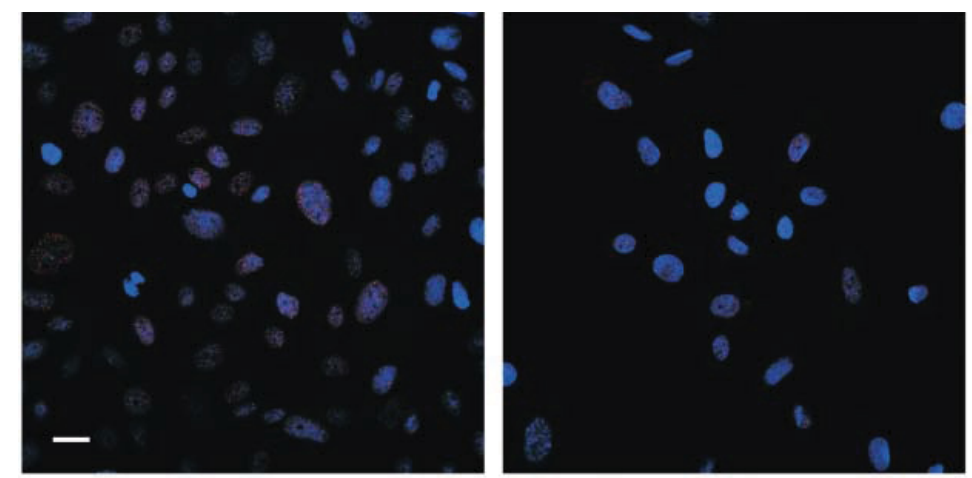

Saos-2-pcDNA 3.1
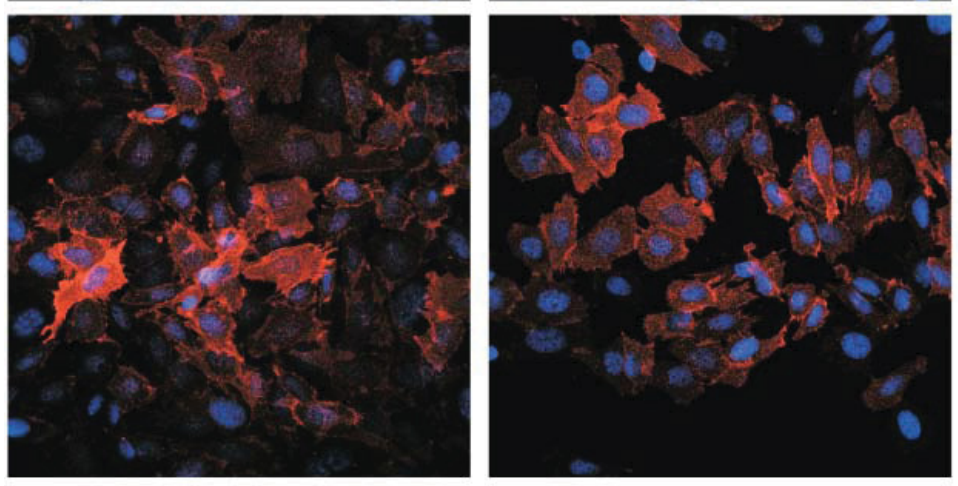

Saos-2-BCRP

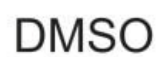

$50 \mu \mathrm{M}$ nutlin-3a

Figure 3-11. Nutlin-3a does not alter cellular localization of BCRP in Saos-2 cells

Confocal images taken of Saos-2 cells expressing BCRP treated with vehicle (DMSO) or nutlin-3a (50 $\mu \mathrm{M}, 90$ minutes). Red represents BCRP protein and blue represents nuclear staining. Scale bar, $30 \mu \mathrm{m}$. Data are representative of two independent experiments. 


\subsubsection{Nutlin-3a is not a substrate for BCRP}

To test whether nutlin-3a is a BCRP substrate and thus inhibits BCRP function by competing with other substrates for transport, we examined the intracellular accumulation of nutlin-3a in MDCKII-pcDNA 3.1 and MDCKII-BCRP cells. Cells were incubated with $0,1,5,10,20$, and $50 \mu \mathrm{M}$ nutlin- $3 \mathrm{a}$ at $37^{\circ} \mathrm{C}$ for 1 hour. No significant difference was observed in the amount of intracellular nutlin-3a between the two cell types at any concentration (up to $50 \mu \mathrm{M})(\mathrm{p}>0.05)$ (Figure 3-12). These data suggest that nutlin-3a is not a substrate of BCRP. In addition to the intracellular accumulation assay, bidirectional transport studies were conducted using BCRP over-expressing and pcDNA3.1 vector control MDCKII cells. Nutlin-3a concentrations from either the apical or basolateral compartment were measured by LC-MS/MS and concentration vs. time plots were generated (Figure 3-13). The calculated $\mathrm{R}\left(\mathrm{RE}_{\mathrm{BCRP}} / \mathrm{RE}_{\mathrm{pcDNA} 3.1}\right)$ was 0.04 , much less than the efflux ratio of 2, which is considered the cutoff for a drug to be a substrate [234]. Additionally, concentration vs. time plots of nutlin-3a in the presence and absence of the BCRP specific inhibitor Ko143 $(5 \mu \mathrm{M})$ indicated that BCRP does not transport nutlin-3a (data not shown). Using the criteria outlined in the decision tree supported by the International Transporter Consortium, nutlin-3a is not a substrate of BCRP [47].

\subsubsection{Nutlin-3a inhibits the ATPase activity of BCRP}

To investigate inhibition as a mechanism for the nutlin-3a-induced reduction in efflux of BCRP substrates, BCRP ATPase activity in response to nutlin-3a treatment was measured using a previously described ATPase activity assay [93, 94]. BCRP transporter activity was determined by assaying both activation and inhibition in the presence of a known activator of the transporter (i.e., sulfasalazine). Increasing concentrations of nutlin-3a $(0.1 \mu \mathrm{M}$ to $150 \mu \mathrm{M})$ did not stimulate ATPase activities from the baseline measurements (data not shown). In the corresponding inhibition assay, however, higher concentrations of nutlin-3a demonstrated a strong capacity to inhibit ATPase activity (Figure 3-14).

\subsection{Discussion}

This is the first study demonstrating that nutlin-3a inhibits BCRP activity. Our data suggest that resistance to mitoxantrone can be strongly reversed by nutlin-3a in BCRP over-expressing cells. Nutlin-3a treatment resulted in a dose-dependent increase in the intracellular accumulation of BCRP substrates in BCRP over-expressing cells. To understand the mechanism behind these observations, a series of studies were performed that clearly demonstrated nutlin-3a inhibited BCRP efflux independent of p53, without altering BCRP protein expression or subcellular localization. Additionally, studies examining the intracellular accumulation of nutlin-3a along with bi-directional transport across MDCKII monolayer cells supported the conclusion that nutlin-3a is not a substrate of BCRP, but does act as an inhibitor through interference with BCRP ATPase activity. 


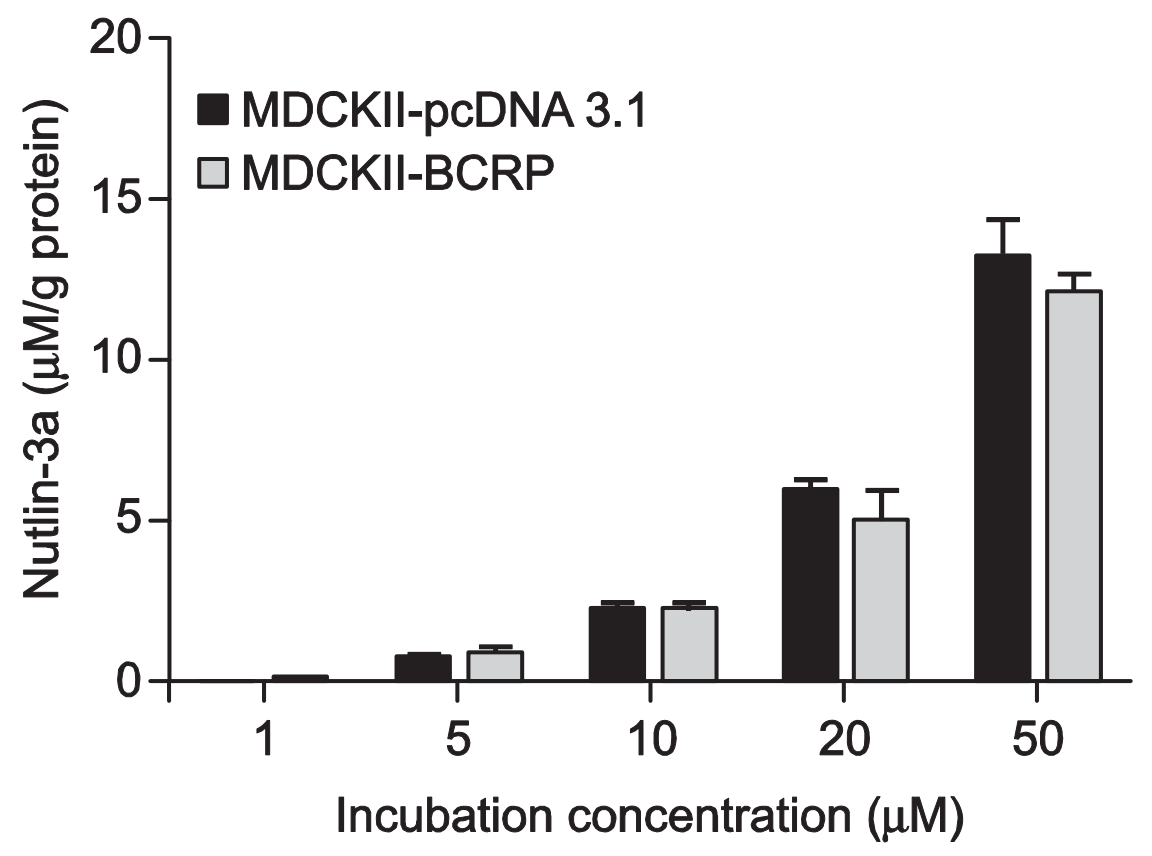

Figure 3-12. No difference in intracellular accumulation of nutlin-3a in the presence of BCRP

MDCKII-pcDNA3.1 and MDCKII-BCRP cells were incubated with 0, 1, 5, 10, 20, and $50 \mu \mathrm{M}$ nutlin-3a for 60 minutes. Intracellular nutlin-3a concentrations were determined by LC-MS/MS as previously described and normalized to total protein content. Data are presented as mean $\pm \mathrm{SD}$ of triplicates. 


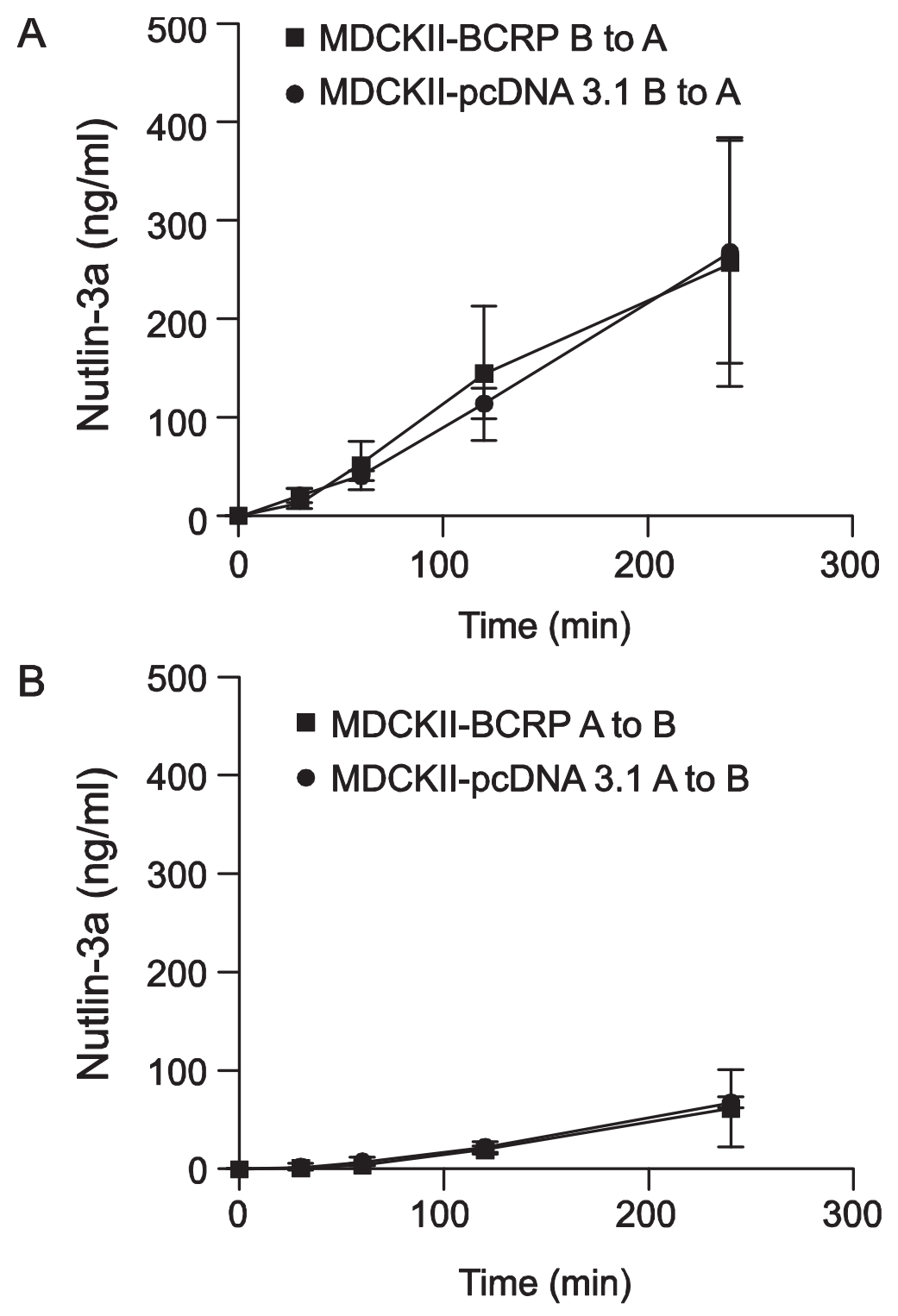

Figure 3-13. Trans-epithelial transport of nutlin-3a $(10 \mu \mathrm{M})$ in MDCKIIpcDNA3.1 and MDCKII-BCRP cells indicates nutlin-3a is not a substrate of BCRP

Nutlin-3a was administered to one compartment (basolateral or apical) at time 0. After $30,60,120$, and 240 minutes, the concentrations of nutlin-3a appearing in the opposite compartment were measured by LC-MS/MS. Data are presented as mean \pm SD of triplicates.

A. Translocation from the basolateral to the apical compartment. Data are representative of two independent experiments.

B. Translocation from the apical to the basolateral compartment. Data are representative of two independent experiments. 


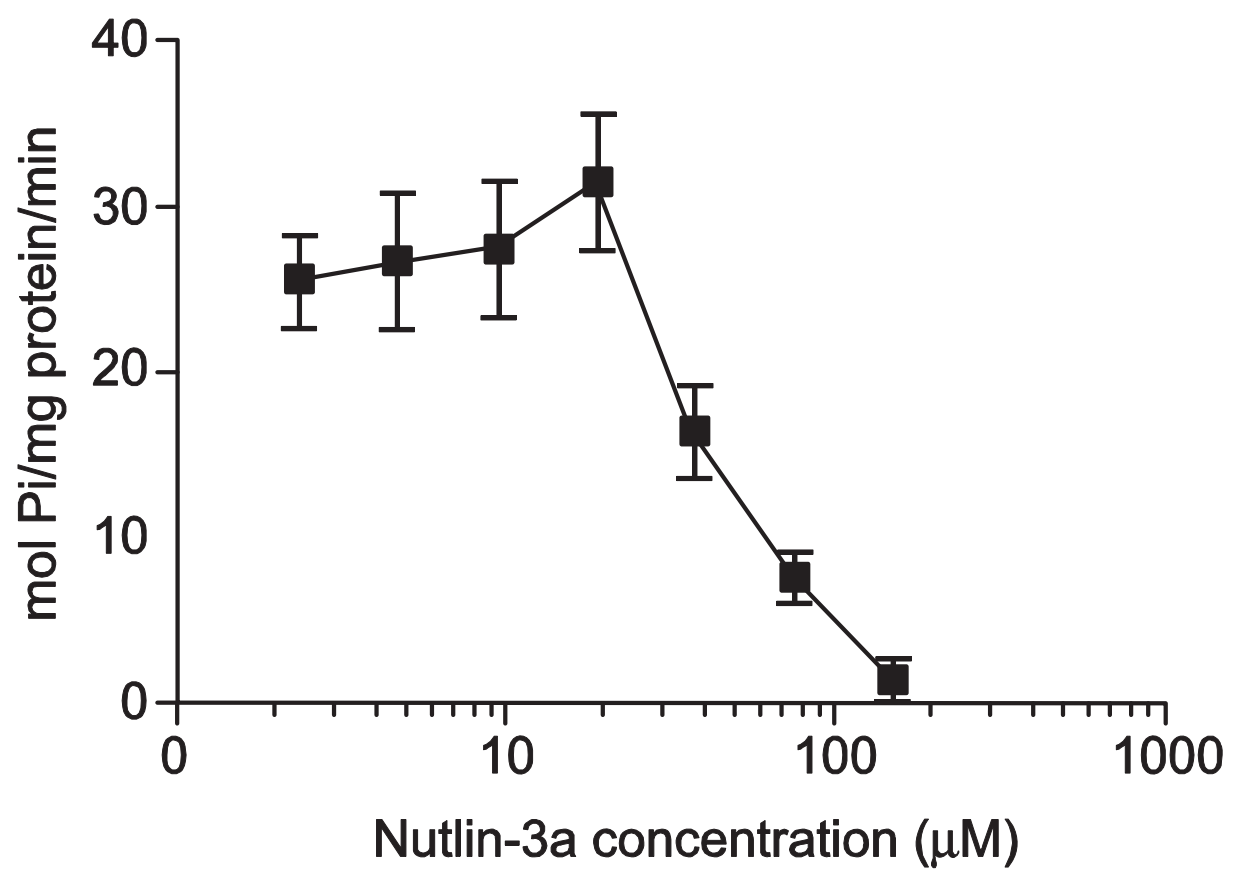

Figure 3-14. Nutlin-3a dose dependently inhibited BCRP ATPase activity

The relative vanadate-sensitive ATPase activity of Sf9 insect cell membranes over-expressing wild-type $A B C G 2$ is represented as $\mathrm{mol} \mathrm{Pi} / \mathrm{mg}$ protein $/ \mathrm{min}$ in the presence of increasing concentrations of nutlin-3a with a known BCRP substrate, sulfasalazine. Data are representative of three independent experiments. 
Multi-drug resistance is a major obstacle in the success of cancer treatment. Among the ABC family of transporters, P-gp, MRP1, and BCRP are three major members associated with multidrug resistance [53]. BCRP, the most recently discovered among these three major transporters $[53,236]$, confers resistance to many anti-cancer drugs used clinically including mitoxantrone, methotrexate, doxorubicin, daunorubicin, topotecan, and SN38 [237, 238]. Utilization of an agent such as nutlin-3a, which inhibits BCRP, in combination with an anti-cancer agent that is a BCRP substrate (such as mitoxantrone or topotecan) may potentially increase the intracellular drug levels and lead to greater anti-tumor activity. In fact, when nutlin-3 was combined with the BCRP and P-gp substrate topotecan for 5 days, an 82-fold reduction in the tumor burden of retinoblastoma was reported [24]. It is important to note however that synergistic effects may differ depending on the cell type or co-administered drug, and antagonism may be observed if the schedule of administration were to change [239, 240].

Since nutlin-3a re-activates p53 in cells co-expressing MDM2 [17, 39], the question exists of whether the reversal of $\mathrm{ABC}$ transporter activity is dependent on the p53 pathway. Our data along with the previous study by Michaelis et al. clearly demonstrate that the inhibition of $\mathrm{ABC}$ transporter efflux by nutlin-3 occurs independently of cellular p53 status [228]. Also supporting this conclusion is the observation that nutlin-3b, the non-active enantiomer, demonstrated BCRP inhibition comparable to the active enantiomer nutlin-3a.

Michaelis et al. demonstrated that nutlin-3 stimulated P-gp ATPase activity in isolated membranes and exerted a negative effect on P-gp activity by acting as a competing substrate [228]. In contrast, our studies demonstrate by multiple approaches that nutlin-3a does not act as a competitive inhibitor of BCRP. First, the amount of intracellular nutlin-3a and the nutlin-3a $\mathrm{IC}_{50}$ were unaffected by the over-expression of BCRP. Second, the calculated efflux ratios from the bidirectional transport assay were $<2$ in MDCKII cells. Lastly, nutlin-3a did not activate ATPase activity as measured by released inorganic phosphate $(\mathrm{Pi})$ in a BCRP over-expression system. On the other hand, nutlin-3a dose dependently decreased the ATPase activity in the inhibition assay.

Previous studies have implicated BCRP translocation from the plasma membrane to the cytoplasm as a mechanism by which BCRP function can be regulated [241]. We demonstrated in our studies via western blot and flow cytometry that neither total BCRP protein levels nor the subcellular localization of BCRP changed during the time period that efflux studies were conducted. These findings are important because since BCRP is involved in drug disposition and many other physiological processes in the body, using a drug that alters BCRP expression and/or localization would likely have global effects. Specifically, BCRP is expressed at the blood brain barrier, placenta, gastrointestinal tract, kidney, liver and biliary tract, and BCRP activity is important for intestinal absorption, brain penetration, renal elimination and hepatobiliary excretion of substrates.

Along with BCRP, P-gp and MRP1 also play a critical role in pharmacokinetic interactions of anti-cancer agents, affecting the absorption, distribution, metabolism, and excretion (ADME) processes. Concomitant treatment of elacridar (GF120918), an 
inhibitor of BCRP and P-gp, resulted in a 2.4 fold increase in bioavailability and systemic exposure of oral topotecan in adults with cancer [242]. Similarly, concomitant treatment with gefitinib, another inhibitor of BCRP and P-gp, increased bioavailability of oral irinotecan in mice [243] and in pediatric patients with refractory solid tumors [244]. Our lab recently demonstrated that gefitinib enhanced topotecan penetration in gliomas in mice [245]. As an inhibitor of multiple major efflux transporters including BCRP, P-gp and MRP1, nutlin-3a may have impact on pharmacokinetics, pharmacodynamics, and importantly the safety of many clinically used drugs. Therefore, it is crucial that transporter related drug-drug interactions be carefully addressed in future preclinical studies.

In conclusion, this is the first study demonstrating that nutlin-3a inhibits BCRP activity. Our data show that nutlin-3a dose dependently inhibits BCRP-mediated transport of multiple BCRP substrates and synergistically reverses the drug resistance to anticancer agent mitoxantrone. The likely mechanism of this effect is the inhibition of BCRP ATPase activity, as we have clearly demonstrated through multiple lines of investigation that nutlin-3a is not a substrate of BCRP. Thus, using nutlin-3a in combination with anti-cancer agents that are BCRP or other $\mathrm{ABC}$ transporter substrates would require additional studies to identify potentially significant drug-drug interactions due to the critical role of these transporters in drug ADME. 


\section{CHAPTER 4. WHOLE-BODY PHYSIOLOGICALLY BASED PHARMACOKINETIC MODEL FOR NUTLIN-3A IN MICE AFTER INTRAVENOUS AND ORAL ADMINISTRATION*}

\subsection{Introduction}

Nutlin-3a (2-piperazinone, 4-[[(4S,5R)-4,5-bis(4-chlorophenyl)-4,5-dihydro-2-[4 methoxy-2-(1-methylethoxy)phenyl]-1H-imidazol-1-yl]carbonyl]-) is currently undergoing preclinical investigation as a p53 reactivation agent. While many cancers and tumor types express mutated forms of p53 [246], a subset of cancers, and particularly pediatric tumors, retain wild-type p53 [211]. In these cases, cancer cells frequently employ other mechanisms to abrogate p53 function. One such mechanism is over-expression or amplification of the murine double minute (MDM2) protein. This molecule binds directly to p53 to accelerate its turnover and inhibits transcription of downstream targets, including cell cycle and apoptotic genes [8, 218]. Disruption of the MDM2-p53 interaction is proposed as a novel strategy for treatment of cancers that do not have p53 alterations [21, 247].

Nutlins are a class of small molecules that target the p53-binding pocket of MDM2 [17, 248]. Treatment of multiple types of cancer cells including leukemias [26, 41], neuroblastoma [221], rhabdomyosarcoma [249] and retinoblastoma [250] with nutlin-3a induces p53-dependent cell cycle arrest and cell death, whereas in normal cells, nutlin-3a exposure leads to cell cycle arrest without cell death [22]. Nutlin-3a has antitumor activity in a preclinical xenograft model of neuroblastoma [43] and was tested in several other preclinical models of malignancies $[17,40]$.

To date, the pharmacokinetics of nutlin-3a has not been reported. An understanding of the systemic disposition of nutlin-3a, as well as the distribution to target tissue or tumor sites, will provide a rational basis for the selection of dosage regimens for preclinical models. Additionally, since the in vitro tumor cell line sensitivities to nutlin-3a have been determined, pharmacokinetic modeling can be used to determine the dose and schedule necessary to achieve appropriate unbound nutlin-3a concentrations at the tumor site. One approach to analyze these data is the use of whole-body physiologically-based pharmacokinetic model (PBPK) models, which are based on anatomical compartments and blood flow.

Thus, we performed pharmacokinetic studies to develop a PBPK model describing the disposition of nutlin-3a in plasma and tissues, including adipose, adrenal gland, bone marrow, brain, liver, lung, intestine, muscle, retina, spleen, and vitreous fluid. The PBPK model was used to perform simulations, which in combination with in vitro cell sensitivity data provided rationale for choosing dosing regimens for mouse

* Reprinted with permission. Zhang, F., et al., Whole-body physiologically based pharmacokinetic model for nutlin-3a in mice after intravenous and oral administration. Drug Metabolism and Disposition, 2011. 39(1): p. 15-21. 
models of common childhood cancers, including retinoblastoma, neuroblastoma, rhabdomyosarcoma, and acute lymphoblastic leukemia (ALL).

\subsection{Materials and Methods}

\subsubsection{Animals}

Adult C57BL/6 mice were purchased from Charles River (Bar Harbor, Maine, USA). Mice were housed in a temperature-controlled room on a normal 12-h light/dark cycle, with free access to water and standard laboratory food. All procedures were approved by the St. Jude Institutional Animal Care and Use Committee and conducted in accordance with the NIH guidelines for the care and use of laboratory animals. The animal facility is accredited by the American Association for Accreditation of Laboratory Animal Care.

\subsubsection{Chemicals}

cis-Nutlin-3a (98\% purity, Lot No.: 08252008) was synthesized and supplied by the Department of Chemical Biology and Therapeutics at St. Jude Children's Research Hospital (Memphis, TN, USA). The oral formulation [17] used in the pharmacokinetic studies was nutlin-3a suspended in 2\% Klucel (Conservation Resources International, LLC, Springfield, VA, USA) and 0.5\% Tween-80 (Sigma-Aldrich, St. Louis, MO, USA), and the IV formulation used was nutlin-3a in 4\% ethanol, 35\% propylene glycol (Fisher Scientific, Pittsburgh, PA, USA), 10\% PEG-400 (Sigma-Aldrich), and 51\% PBS (Mediatech INC, Manassas, VA, USA). The internal standard ketoconazole was purchased from Sigma-Aldrich. Blank murine plasma was obtained from Hilltop Lab Animals, Inc. (Scottsdale, PA, USA). All other reagents were of analytical grade or higher.

\subsubsection{Blood to plasma ratio}

Pooled whole blood from healthy male C57BL/6 mice was collected into heparin tubes. Nutlin-3a was spiked into aliquots of whole blood at final concentrations of $0.1,1$, 10 , and $100 \mu \mathrm{M}$ in triplicate. Samples were mixed and incubated at $37^{\circ} \mathrm{C}$ for 30 minutes with additional mixing every 5 minutes. After incubation, $50 \mu 1$ of whole blood was removed and immediately placed on dry ice. The remainder of the sample was centrifuged at 16,000 rpm for 2 min and a $50 \mu \mathrm{l}$ plasma sample was removed and immediately placed on dry ice. All samples were stored at $-80^{\circ} \mathrm{C}$ until analysis. The blood to plasma concentration ratio was calculated using the using Equation 4-1:

$$
\text { Blood to plasma ratio }=\frac{C_{W B}}{C_{p}}
$$


where $\mathrm{C}_{\mathrm{P}}$ is the concentration in plasma, $\mathrm{C}_{\mathrm{WB}}$ is the concentration in whole blood.

\subsubsection{Nutlin-3a protein binding studies}

Equilibrium dialysis was performed in a 96-well dialysis plate with a 5-KDa cut-off membrane (Harvard Apparatus, Holliston, MA, USA). For mouse plasma and cell culture media protein binding, $200 \mu 1$ of PBS buffer was added into the wells on one side of the membrane and an equivalent volume of male C57BL/6 plasma or cell culture media (RPMI with 10\% FBS and $2 \mathrm{mM}$ L-glutamine) containing varying concentration of nutlin-3a was added into the wells on the opposite side. The plate was sealed and fixed onto a dual plate rotator (Harvard Apparatus) at $37^{\circ} \mathrm{C}$ in a humidified incubator containing $5 \% \mathrm{CO}_{2}$. Equilibrium dialysis was performed at $0.2,20$, and $100 \mu \mathrm{M}$ nutlin-3a in triplicate for $24 \mathrm{~h}$. For vitreous protein binding, $150 \mu \mathrm{l}$ rodent vitreous containing 0.5 $\mu \mathrm{M}$ nutlin-3a was added to the sample side and equal volume of PBS buffer was added to the buffer side. Equilibrium dialysis was performed in triplicate for $24 \mathrm{~h}$. The samples were analyzed using the analytical method described below. The bound concentration was considered equal to the plasma side and the free concentration equal to the PBS side $[251,252]$. Binding parameters were estimated with nonlinear regression using Equation 4-2 [253]:

$$
C_{p, b}=\frac{B_{\max } \cdot K_{A} \cdot C_{p, f}}{1+K_{A} \cdot C_{p, f}}
$$

where $\mathrm{C}_{\mathrm{p}, \mathrm{b}}$ is the bound plasma concentration, $\mathrm{B}_{\max }$ is the quantity of plasma protein binding sites, $\mathrm{K}_{\mathrm{A}}$ is the binding association constant, and $\mathrm{C}_{\mathrm{P}, \mathrm{f}}$ is the unbound plasma concentration.

\subsubsection{Drug administration and sample collection}

Two pharmacokinetic studies were conducted. For the first pharmacokinetic study, 145 adult C57BL/6 mice (128 male and 17 female) were divided into three groups: two oral dosage groups (100 and $200 \mathrm{mg} / \mathrm{kg}$ ) and one IV dosage group (10 mg/ $\mathrm{kg}$ ). Nutlin-3a was administered given as a single bolus dose by oral gavage or by i.v. tail vein injection. Each dosing group $(\mathrm{n}=5$ mice $)$ and vehicle controls had 9 collection time points $(0.5,1,2,4,8,12,24,36$, and $48 \mathrm{~h})$. At each time point, blood was collected under isoflurane anesthesia via cardiac puncture. Whole blood samples were centrifuged immediately at $12,000 \mathrm{~g}$ at $4{ }^{\circ} \mathrm{C}$ for 5 minutes to separate plasma. Simultaneously, tissue samples including brain, vitreous, retina, liver, spleen, and bone marrow were dissected. Each sample was put on dry ice immediately after collection and stored at $-80^{\circ} \mathrm{C}$ until analysis.

In the second pharmacokinetic study, 210 adult male C57BL/6 mice were used. Two oral dosages (50 and $100 \mathrm{mg} / \mathrm{kg}$ ) and two i.v. dosages (10 and $20 \mathrm{mg} / \mathrm{kg}$ ) were administered. Each dosing group $(\mathrm{n}=5$ for $10 \mathrm{mg} / \mathrm{kg} \mathrm{IV}$ and $100 \mathrm{mg} / \mathrm{kg}$ oral dosages; 
$\mathrm{n}=10$ for $20 \mathrm{mg} / \mathrm{kg} \mathrm{IV}$ and $50 \mathrm{mg} / \mathrm{kg}$ oral dosages) and vehicle control had 7 collection time points $(0.5,1,2,4,8,12$, and $24 \mathrm{~h}$ for the IV dosing; $0.5,1,2,4,8,12$, and $16 \mathrm{~h}$ for oral dosing). Serial plasma samples were collected from all mice. Tissue samples, including brain, lung, liver, spleen, kidney, adrenal gland, muscle, fat, intestine from 3 mice per time point from the $20 \mathrm{mg} / \mathrm{kg}$ IV group, were collected. Each sample was put on dry ice immediately after the sample collection and stored at $-80^{\circ} \mathrm{C}$ until analysis.

\subsubsection{Quantitative analysis of nutlin-3a}

Nutlin-3a mouse plasma and tissue samples were analyzed based on our previously published liquid chromatography electrospray ionization tandem mass spectrometry analytical method [233]. The lower limit of quantification (LLOQ) for nutlin-3a in plasma after protein precipitation was $10 \mathrm{ng} / \mathrm{ml}$ and the LLOQ for nutlin-3a after liquid-liquid extraction was $0.25 \mathrm{ng} / \mathrm{mL}$. Within-day and between-day precisions for protein precipitation and liquid-liquid extraction were $\leq 5 \%$ and accuracies ranged from 91.6 to $104.8 \%$. For each sample type (cerebellum, brain, vitreous, retina, lung, heart, liver, gall bladder, spleen, kidney, adrenal gland, muscle, fat, bone marrow, intestine, whole blood, and PBS), standard curves and controls were generated using the corresponding untreated tissue or PBS to eliminate any matrix effect. For larger tissues, sections were cut, weighed, and stored on ice for further processing. $10 \mu \mathrm{L}$ ice cold homogenization buffer $(5 \mathrm{mM} \mathrm{HCOONH} 4, \mathrm{pH}=7)$ were added per $\mathrm{mg}$ of tissue. For smaller tissue samples including vitreous, retina, adrenal gland, and gall bladder, the amount of homogenization buffer used was increased to a minimum volume of 70 100 $\mu \mathrm{L}$. Tissue samples were then sonicated on ice for 15 seconds, with 5 second intervals. The number of total sonications varied depending on the tissue types. Homogenated tissues and whole blood samples were extracted and analyzed using protein precipitation and the PBS samples were extracted using the liquid-liquid extraction method as described previously [233].

\subsubsection{Whole body PBPK model development}

We developed a whole body PBPK model for nutlin-3a based on in vitro blood cell partitioning, plasma protein binding, and pooled concentration-time data from all plasma and tissue samples collected from both pharmacokinetic studies. This PBPK model consisted of a series of mass balance differential equations describing the concentration of nutlin-3a in various tissues, which were connected by blood flow. Physiological values for mouse organ size and blood flow are presented in Table 4-1. A schematic representation of the model is shown in Figure 4-1.

Plasma concentrations were converted to whole blood concentrations based on the in vitro blood partitioning experiment. Unbound plasma concentrations were described with Equation 4-3 [253]: 
Table 4-1. List of physiological parameters

\begin{tabular}{ccccc}
\hline Tissue & Symbol & $\begin{array}{c}\text { Mass }(\% \\
\text { body weight })\end{array}$ & $\mathrm{Q}_{\text {В }}(\mathrm{ml} / \mathrm{h})$ & Reference \\
\hline Blood & BLO & 4.9 & 839 & {$[254]$} \\
Adipose & ADI & 6.8 & 58.7 & {$[254]$} \\
Adrenal gland & ADR & 0.048 & 2.52 & {$[254]$} \\
Bone marrow & MRW & 5.8 & 92.3 & {$[254]$} \\
Brain & BRA & 1.65 & 27.7 & {$[254]$} \\
Intestines & INT & 3.62 & 109 & {$[254]$} \\
Liver & LIV & 5.49 & 16.8 & {$[254]$} \\
Lung & LUN & 0.73 & 839 & {$[254]$} \\
Muscle & MUS & 38.4 & 133 & {$[254]$} \\
Retina & RET & 0.04 & 3.16 & Experimental; [255] \\
Spleen & SPL & 0.35 & 9.48 & - \\
Vitreous fluid & VIT & 0.035 & $0 * 2$ & Extal \\
Remainder & RES & 29.9 & 256.9 & \\
\hline
\end{tabular}

* Vitreous assumed to have no direct blood flow. 


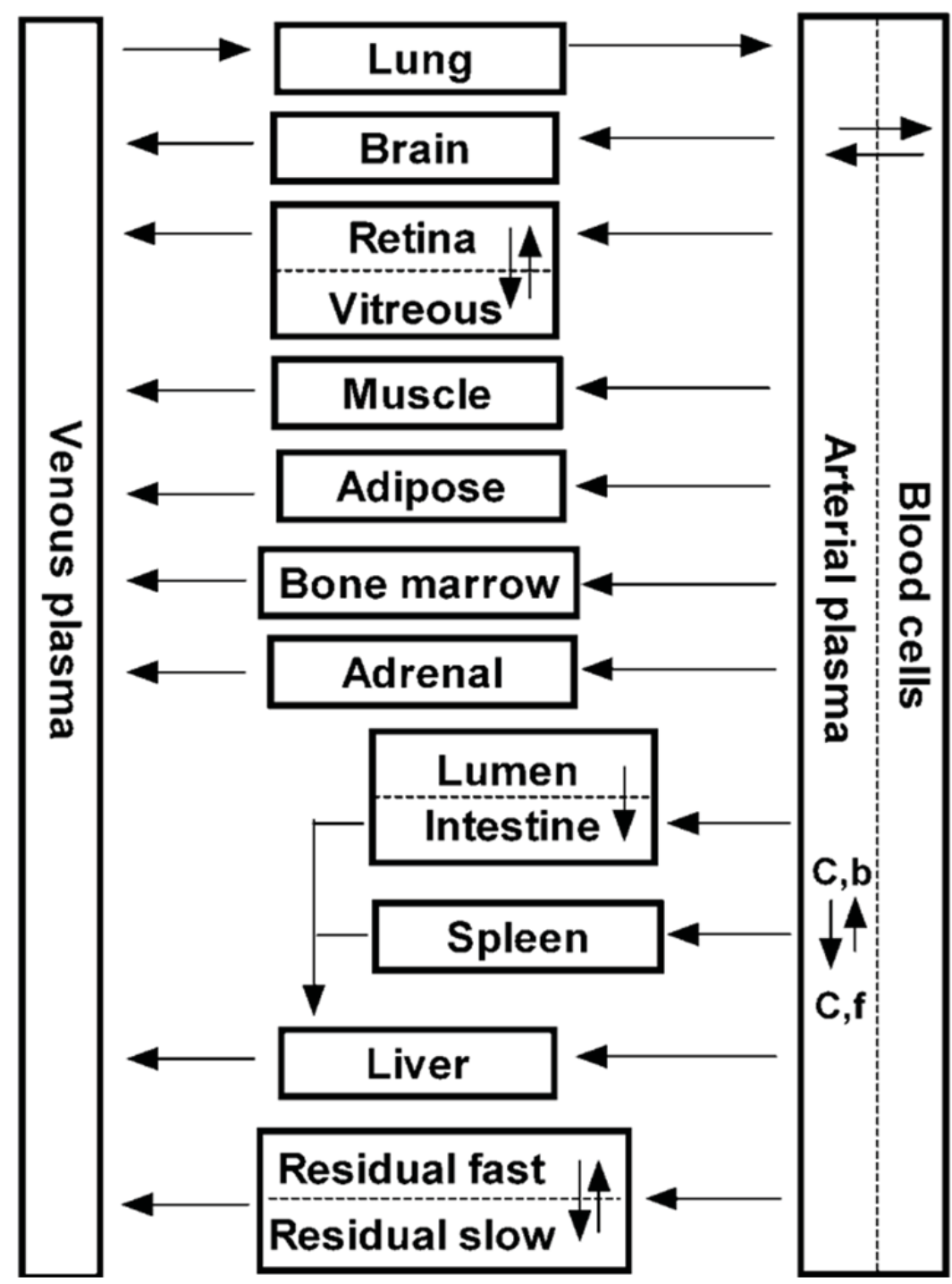

Figure 4-1. Schematic diagram of PBPK model for nutlin-3a in mice

$\mathrm{C}, \mathrm{b}=$ bound drug concentration, $\mathrm{C}, \mathrm{f}=$ free drug concentration. Arrows connecting compartments represent blood flows from literature values. 


$$
C_{p . u}=\frac{-\left(1+B_{\max }-K_{A} \cdot C_{p}\right)+\left\{\left(1+B_{\max }-K_{A} \cdot C_{p}\right)^{2}+4 \cdot K_{A} \cdot C_{p}\right\}^{1 / 2}}{2 \cdot K_{A}}
$$

where $\mathrm{C}_{\mathrm{p}, \mathrm{u}}$ is the unbound plasma concentration, $\mathrm{C}_{\mathrm{p}}$ is the total plasma concentration, and $\mathrm{B}_{\max }$ and $\mathrm{K}_{\mathrm{A}}$ were determined from in vitro plasma protein binding studies. The unbound fraction ( $\left.f_{u b}\right)$ was calculated by Equation 4-4:

$$
f_{u b}=\frac{C_{p, u}}{C_{p}}
$$

Eq. 4-4

Most organs fit well to a perfusion-limited model, and thus were described by

\section{Equation 4-5:}

$$
V_{i} \cdot \frac{d A_{i}}{d_{t}}=Q_{i} \cdot\left(C_{A R T}-\frac{C_{i}}{K_{i}}\right)
$$

where $\mathrm{V}_{\mathrm{i}}$ is the volume of organ, $\mathrm{A}_{\mathrm{i}}$ is the amount of drug in the organ, $\mathrm{C}_{\mathrm{i}}$ is the concentration in the organ, $\mathrm{K}_{\mathrm{i}}$ is the partition coefficient, and $\mathrm{C}_{\mathrm{ART}}$ is the concentration of arterial plasma.

Liver blood flow (QLIV) was the sum of the blood flow to the hepatic artery, spleen, and liver, and the concentration of blood entering the liver ( $\mathrm{C}_{\mathrm{BLO}}$, LIV $)$ was based on the arterial concentration and the venous outflow of the portal circulation (Equation 4-6). The liver contained an elimination term $\left(\mathrm{k}_{\mathrm{e}}\right)$ for metabolism, based on experiments showing that nutlin-3a is metabolized by mouse liver microsomes (K. Guy, unpublished):

$$
V_{L I V} \cdot \frac{d A_{L I V}}{d_{t}}=Q_{L I V} \cdot\left(C_{B L O, L I V}-\frac{C_{L I V}}{K_{L I V}}\right)-k_{e} \cdot C_{A R T}
$$

The intestine was modeled with a separate lumen and tissue compartment. Absorption from the lumen was assumed to be linear based on an absorption rate constant $\left(\mathrm{k}_{\mathrm{a}}\right)$ (Equation 4-7):

$$
V_{I N T} \cdot \frac{d A_{I N T}}{d_{t}}=k_{a} \cdot A_{I N T}
$$

The eye was fit to a two-compartment model consisting of the retina and vitreous. Input into the vitreous was by diffusion from the retina. The following equations were used for the retina and vitreous (Equation 4-8 and Equation 4-9):

$$
\begin{array}{cc}
V_{R E T} \cdot \frac{d A_{R E T}}{d_{t}}=Q_{R E T} \cdot\left(C_{A R T}-\frac{C_{R E T}}{K_{R E T}}\right)-P A_{V I T} \cdot\left(C_{R E T}-\frac{C_{V I T}}{K_{V I T}}\right) & \text { Eq. 4-8 } \\
V_{V I T} \cdot \frac{d A_{V I T}}{d_{t}}=P A_{V I T} \cdot\left(C_{R E T}-\frac{C_{V I T}}{K_{V I T}}\right) & \text { Eq. 4-9 }
\end{array}
$$

where PA $\mathrm{VIT}_{\text {is }}$ the permeability-surface area product. 
All tissues that were not sampled were lumped together in a residual compartment. Modeling this compartment as perfusion-limited did not adequately describe the multi-exponential profile of nutlin-3a. Therefore, the residual compartment was modeled as diffusion-limited, with the vascular space assumed to be $5 \%$ of the residual volume. The equations for the residual vascular space and tissue are the described using Equation 4-10 and Equation 4-11:

$$
\begin{aligned}
& V_{R E S, B L O} \cdot \frac{d A_{R E T}}{d_{t}}=Q_{R E S} \cdot\left(C_{A R T}-C_{R E S, B L O}\right)-P A_{R E S} \cdot\left(C_{R E S}-\frac{C_{R E S}}{K_{R E S}}\right) \text { Eq. 4-10 } \\
& V_{R E S} \cdot \frac{d A_{R E S}}{d_{t}}=P A_{R E S} \cdot\left(C_{R E S}-\frac{C_{R E S}}{K_{R E S}}\right)
\end{aligned}
$$

The input into the venous pool of blood was modeled as the sum of the output from all tissues except the lung. The volume of the venous pool was fixed to $75 \%$ of the total blood volume. The lungs received all venous input and the arterial input was the output from the lungs (Equation 4-12):

$$
V_{L U N} \cdot \frac{d A_{L U N}}{d_{t}}=Q_{B L O} \cdot\left(C_{V E N}-\frac{C_{L U N}}{K_{L U N}}\right)
$$

The arterial concentrations were based on the output from the lungs, and contained an additional saturable elimination term (Equation 4-13):

$$
V_{A R T} \cdot \frac{d A_{A R T}}{d_{t}}=Q_{B L O} \cdot\left(\frac{C_{L U N}}{K_{L U N}}-C_{A R T}\right)-\frac{V_{\max } \cdot C_{A R T}}{K_{m}+C_{A R T}}
$$

Elimination terms in both the blood compartment and liver compartment were necessary for a good model fit to the data from both oral and intravenous administration.

\subsubsection{Simulations}

After development of the PBPK model, tissue concentrations were simulated with NONMEM after multiple oral and intravenous doses at 50,100,200, and $400 \mathrm{mg} / \mathrm{kg}$ given both once daily and twice daily. The $\mathrm{AUC}_{0-24}$ at steady state was calculated with the log-linear trapezoidal method applied to the simulated data. Bioavailability was estimated using the ratio of $\mathrm{AUC}_{0-\tau}, \mathrm{IV}_{\mathrm{V}} / \mathrm{AUC}_{0-\tau}$, PO with the simulated steady state data.

\subsection{Results}

\subsubsection{Blood to plasma partitioning and plasma protein binding of nutlin-3a}

Blood to plasma partitioning showed an average blood/plasma concentration ratio of 0.70 , indicating that $30 \%$ of nutlin-3a partitions to blood cells (Figure 4-2A). Binding of nutlin-3a to mouse plasma proteins was nonlinear, with $\mathrm{f}_{\mathrm{ub}}$ ranging from 0.007 at 0.1 

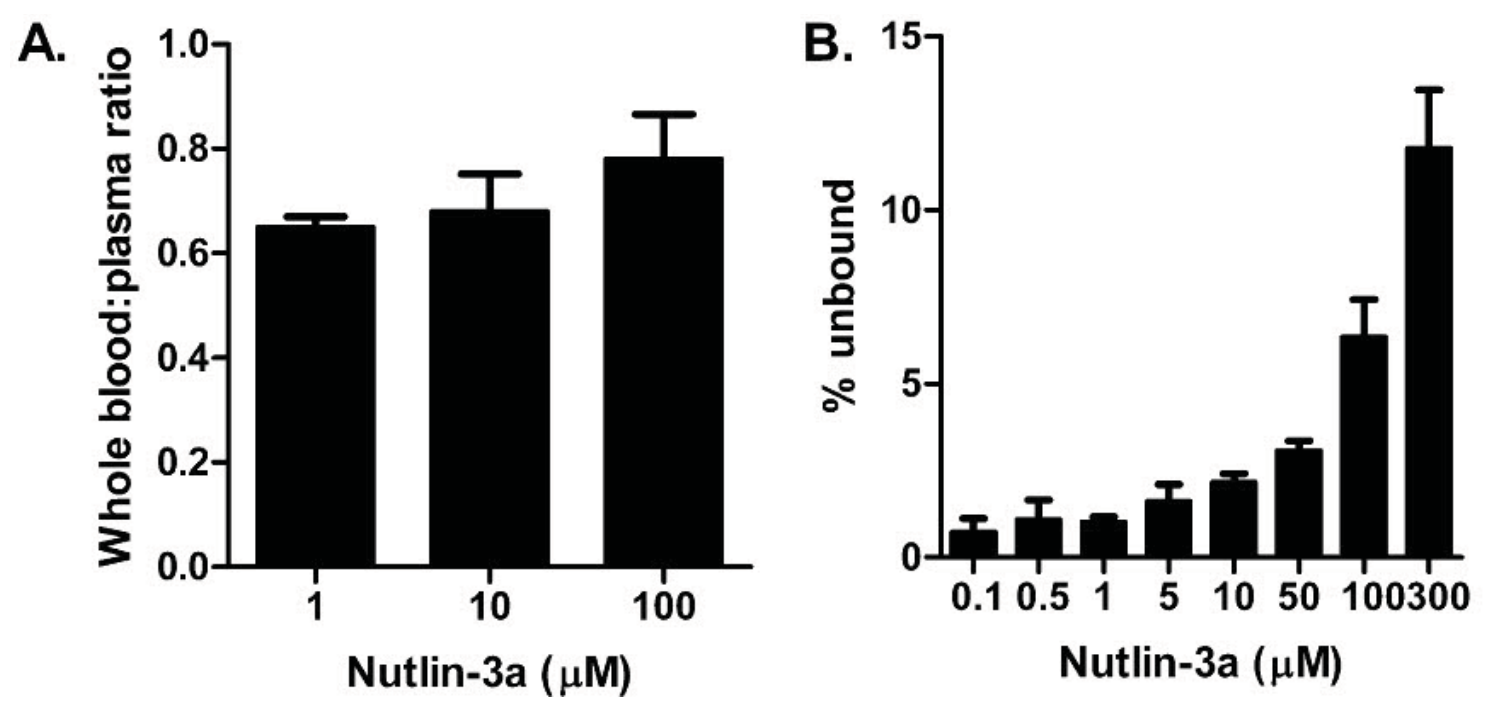

Figure 4-2. Analysis of nutlin-3a characteristics in murine blood

\begin{abstract}
A. Nutlin-3a blood cell partitioning. Nutlin-3a was spiked into murine whole blood at various concentrations, and incubated $30 \mathrm{~min}$ at $37^{\circ} \mathrm{C}$. In one aliquot, nutlin-3a was measured in whole blood, and in another aliquot nutlin-3a was measured in the plasma.

B. Nutlin-3a plasma protein binding. Nutlin-3a was spiked into murine plasma at various concentrations and incubated for $30 \mathrm{~min}$ at $37^{\circ} \mathrm{C}$. Plasma protein binding was determined by equilibrium microdialysis and is expressed as the percent of the total nutlin-3a plasma concentration that is unbound. Bars represent the mean and error bars represent the standard deviation.
\end{abstract}


$\mu \mathrm{M}$ to 0.118 at $300 \mu \mathrm{M}$ (Figure 4-2B). Nonlinear regression of unbound versus bound plasma concentrations using the Langmuir equations resulted in a Bmax of 286 and a KA of 0.085 (Figure 4-3).

\subsubsection{Nutlin-3a pharmacokinetics in mice}

Plasma and tissue concentrations of nutlin-3a were measured from 0 to $48 \mathrm{~h}$ in mice following a single i.v. dose of 10 or $20 \mathrm{mg} / \mathrm{kg}$ or a single oral dose of 50,100 , or $200 \mathrm{mg} / \mathrm{kg}$. After oral administration, nutlin-3a tissue concentrations rose rapidly to reach a maximum value at approximately $2 \mathrm{~h}$. Nutlin-3a concentrations in the intestine, liver, and spleen were higher than those in the plasma, concentrations in adipose, adrenal gland, lung, muscle, and retina were similar to plasma concentrations, and concentrations in the brain, bone marrow, and vitreous were significantly lower than in the plasma (Figure 4-4).

Rapid elimination was observed in the $10 \mathrm{mg} / \mathrm{kg}$ IV dosage group. At higher dosages, slower elimination was observed at higher concentrations, indicating saturable elimination of nutlin-3a. After $24 \mathrm{~h}$, all data were below the limit of quantitation of the assay. Models with linear elimination, Michaelis-Menten elimination, and combined linear and Michaelis-Menten elimination were fit to the data. Ultimately, the combination of both linear and Michaelis-Menten elimination had the best fit to the data. The concentration-time data of nutlin-3a in all modeled tissues are plotted against the model predicted concentrations in Figure 4-5 (data not shown for oral $50 \mathrm{mg} / \mathrm{kg}$ dosage group, since only plasma was collected). The estimated pharmacokinetic parameters are listed in Table 4-2.

Using the final model, we simulated nutlin-3a plasma concentrations after multiple doses on a once-daily and twice-daily schedule (Figure 4-6). Little to no accumulation was predicted to occur on a once daily schedule with IV or oral dosages up to $400 \mathrm{mg} / \mathrm{kg}$. For twice daily dosing, steady state was predicted to occur within three doses, but accumulation would remain minimal. The predicted accumulation $\left(\mathrm{C}_{\min }\right.$ single dose $/ \mathrm{C}_{\min }$ at steady state) was dose-dependent and at $200 \mathrm{mg} / \mathrm{kg}$ is 1.5 -fold for IV administration and 1.3-fold for oral administration. The $\mathrm{AUC}_{0-24}$ at steady state increased in an approximately dose-proportional manner and the AUC0-24 was approximately twice as high with twice-daily dosing versus once-daily dosing (Figure 4-7).

Predicted bioavailability was dose- and schedule -dependent and ranged from $75 \%$ at $25 \mathrm{mg} / \mathrm{kg}$ once-daily to $91 \%$ at $400 \mathrm{mg} / \mathrm{kg}$ once-daily. Bioavailability was predicted to be near $100 \%$ when given twice daily at dosages of $50 \mathrm{mg} / \mathrm{kg}$ or higher. 


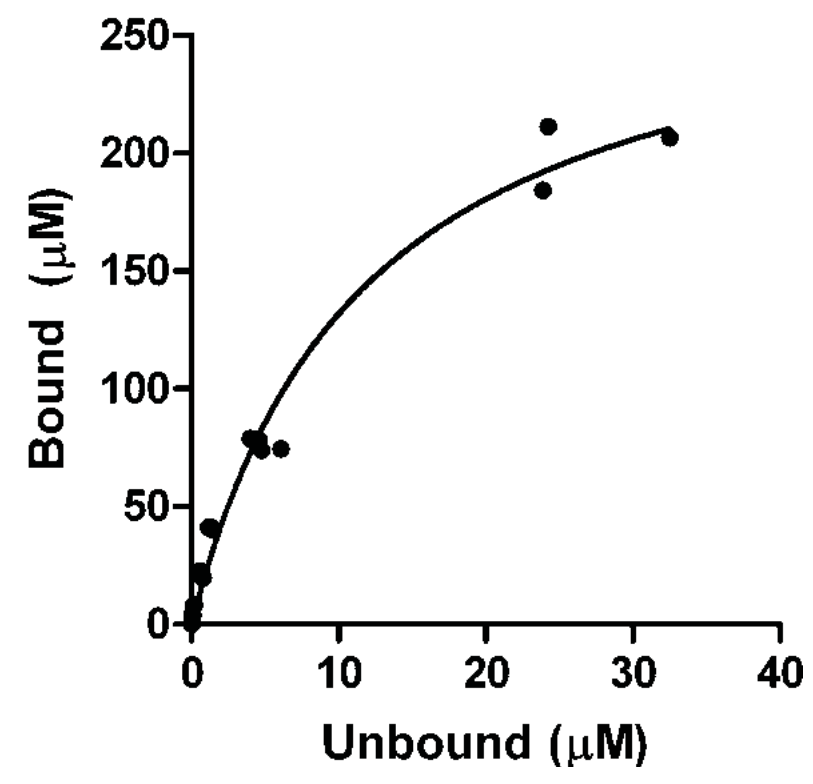

Figure 4-3. Nutlin-3a binding to murine plasma proteins

Bound and unbound nutlin-3a plasma concentrations were determined with equilibrium dialysis and nonlinear regression was performed using the Langmuir equation. 


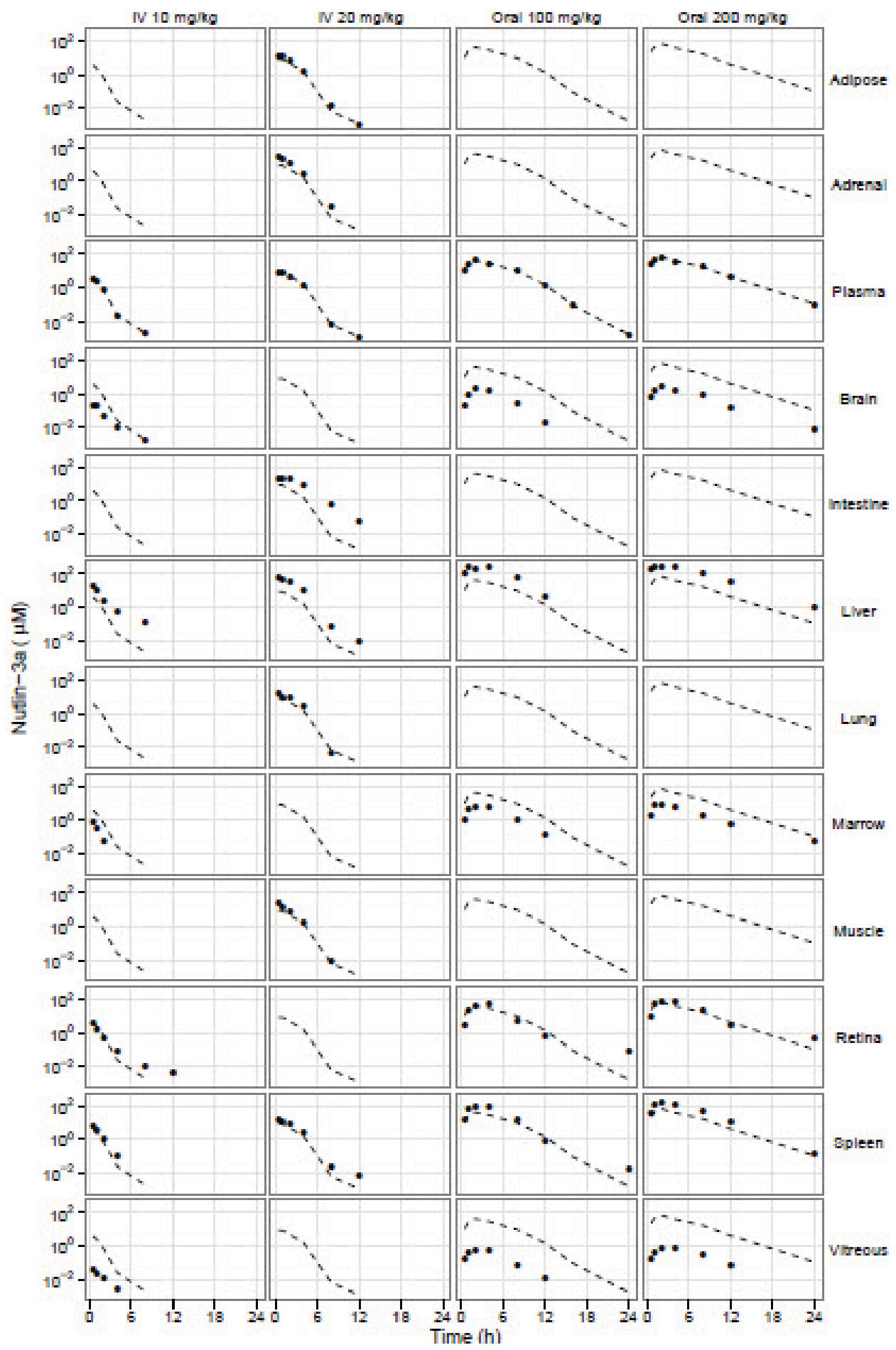

Figure 4-4. Comparison of actual plasma and tissue concentrations of nutlin-3a

The median plasma concentrations are shown in each box as the dashed line and the symbols represent the median tissue concentrations. 


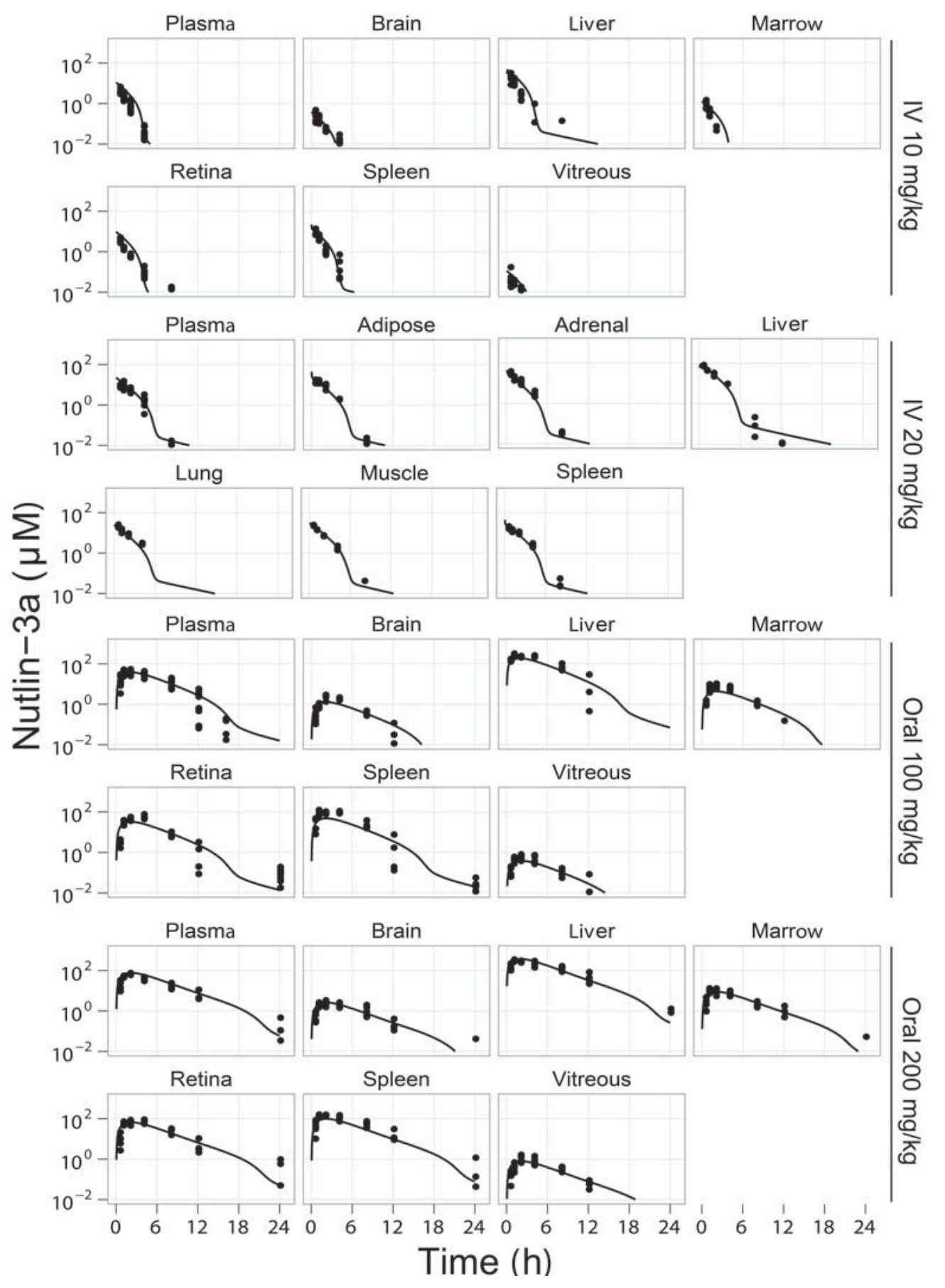

Figure 4-5. Concentration-time plots of nutlin-3a in tissues

Symbols are data points from individual mice and the lines represent the model-predicted concentrations. Data from the $50 \mathrm{mg} / \mathrm{kg}$ oral group is not shown. Data below the lower limit of quantitation is not shown. 
Table 4-2. Estimated PBPK model parameters

\begin{tabular}{|c|c|}
\hline Parameter & Value \\
\hline$k_{\mathrm{a}}$ & 0.409 \\
\hline$K_{\mathrm{e}}$ & 0.0160 \\
\hline $\mathrm{V}_{\max }$ & 0.0287 \\
\hline $\mathrm{K}_{\mathrm{m}}$ & 0.050 \\
\hline $\mathrm{K}_{\mathrm{ADI}}$ & 1.61 \\
\hline $\mathrm{K}_{\mathrm{ADR}}$ & 2.05 \\
\hline $\mathrm{K}_{\mathrm{BRA}}$ & 0.055 \\
\hline $\mathrm{K}_{\mathrm{INT}}$ & 12.2 \\
\hline $\mathrm{K}_{\mathrm{LIV}}$ & 7.54 \\
\hline $\mathrm{K}_{\text {LUN }}$ & 1.78 \\
\hline $\mathrm{K}_{\text {MUS }}$ & 2.08 \\
\hline $\mathrm{K}_{\mathrm{RET}}$ & 4.01 \\
\hline $\mathrm{K}_{\mathrm{SPL}}$ & 2.72 \\
\hline $\mathrm{K}_{\mathrm{VIT}}$ & 0.012 \\
\hline $\mathrm{PA}_{\mathrm{VIT}}$ & 0.0036 \\
\hline $\mathrm{K}_{\mathrm{RES}}$ & 4.8 \\
\hline $\mathrm{PA}_{\mathrm{RES}}$ & 0.0048 \\
\hline IIV $\mathrm{k}_{\mathrm{a}}$ & $31.2 \%$ \\
\hline IIV $k_{\mathrm{e}}$ & $6.4 \%$ \\
\hline IIV $V_{\text {max }}$ & $40.6 \%$ \\
\hline Residual error & $35.6 \%$ \\
\hline
\end{tabular}




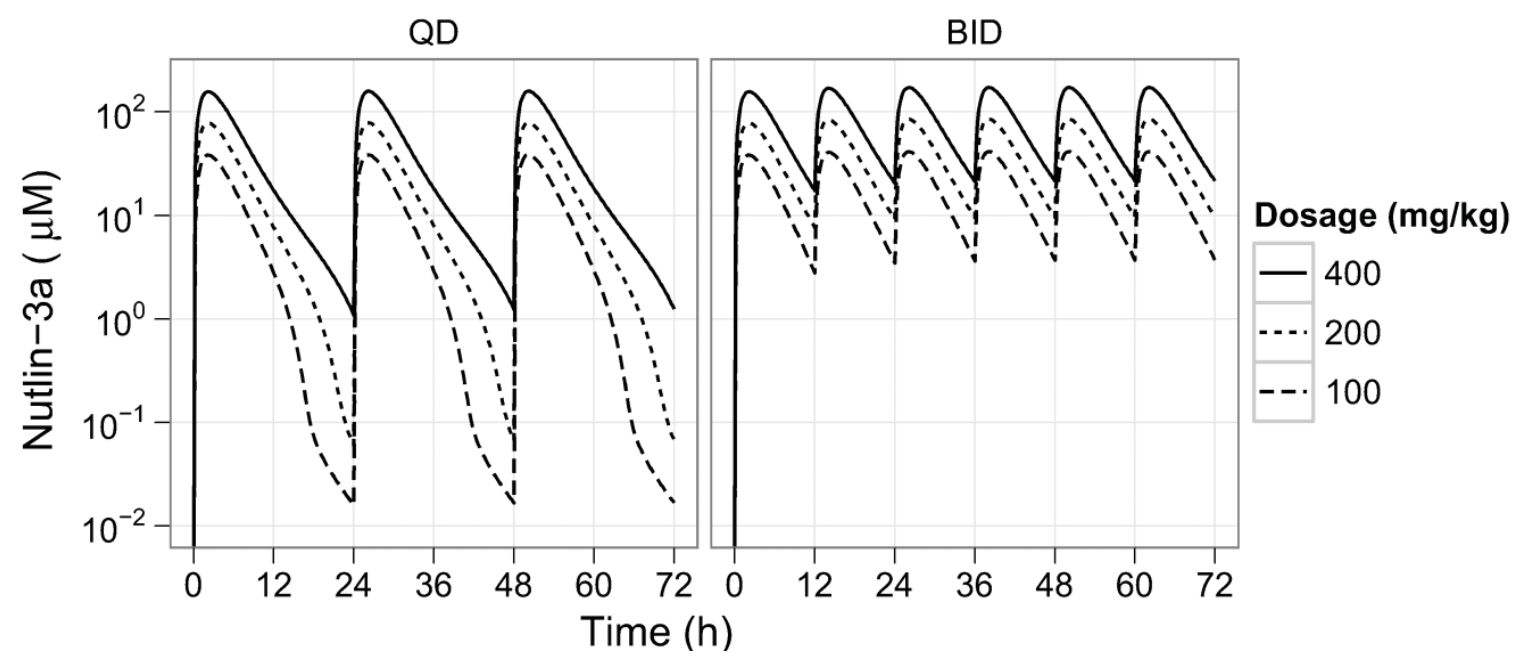

Figure 4-6. Simulated concentration-time plot of plasma nutlin-3a after multiple oral doses with once-daily (QD) and twice-daily (BID) dosing

Simulations were based on the final PBPK model. 


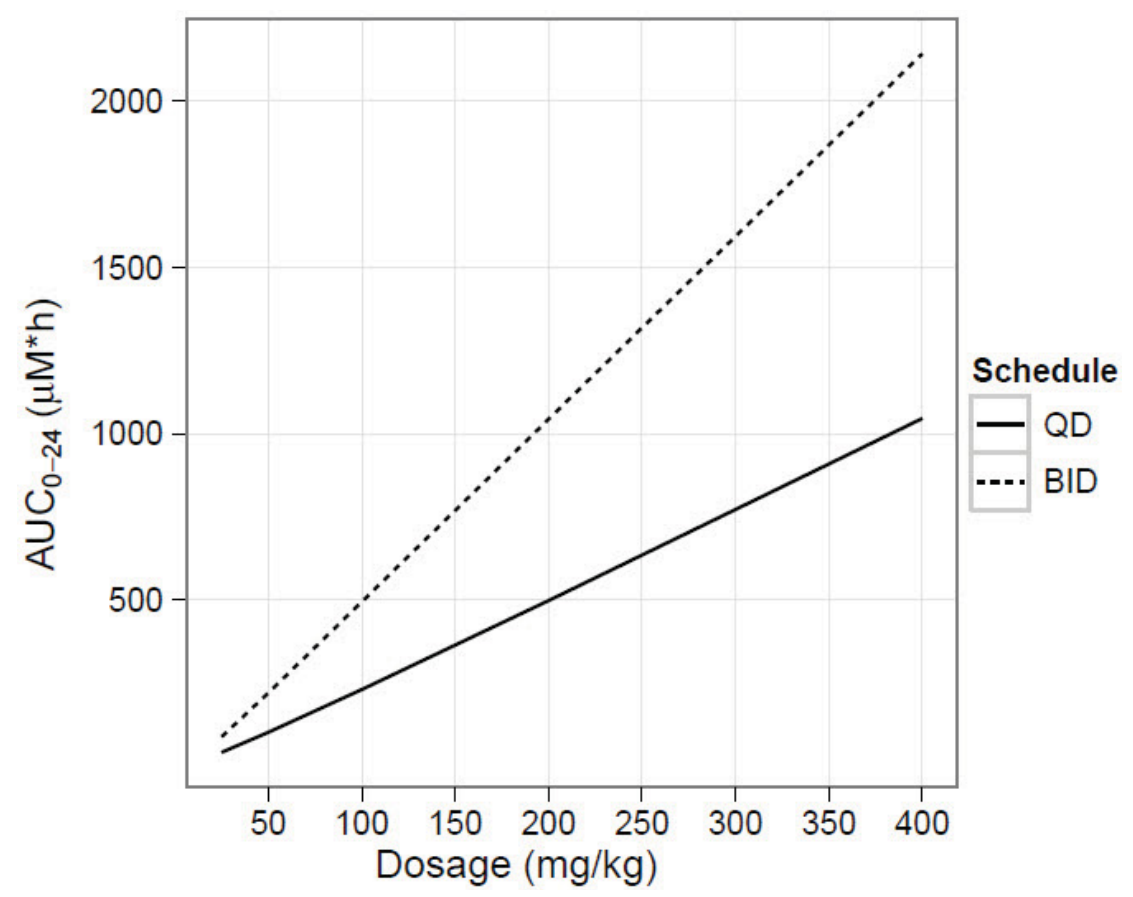

Figure 4-7. Plasma area under the concentration-time curve for $24 \mathrm{~h}$ at steady state (AUC0-24) versus nutlin-3a dosage when administered once daily (QD) and twice daily (BID)

AUCs were calculated from simulated concentration-time curves based on the final PBPK model. 


\subsubsection{Application of PBPK model to the design of nutlin-3a dosing regimens in mice}

The nutlin-3a PBPK model was used to choose dosing regimens of nutlin-3a to target (1) the retina and vitreous for models of retinoblastoma, (2) the adrenal gland for models of neuroblastoma, (3) the muscle for models of rhabdomyosarcoma, and (4) the plasma, spleen, and bone marrow for models of leukemia. The fraction of unbound nutlin-3a in tissues was assumed to be the same as the plasma unbound fraction, except for vitreous fluid that had a measured unbound fraction of $14.4 \%$. Nutlin-3a binding to cell culture media was measured and shown to be nonlinear over the range of nutlin-3a concentrations used in in vitro cell cytotoxicity assays (Figure 4-8). The media protein binding value was used to convert the published nutlin-3a $\mathrm{IC}_{50}$ values of various cell lines $[26,249,256]$ to the unbound $\mathrm{IC}_{50}$ (Table 4-3).

The simulated unbound retina and vitreous nutlin-3a concentrations were compared to the in vitro unbound $\mathrm{IC}_{50}$ for the Weri1 retinoblastoma cell line in order to choose optimal dosing regimens for mouse models of retinoblastoma (Figure 4-9). Oral dosing of nutlin-3a twice daily was predicted to achieve unbound concentrations in the retina that were consistently above the unbound $\mathrm{IC}_{50}$ at dosages of 200 or $400 \mathrm{mg} / \mathrm{kg}$ (Table 4-4). However, even at $400 \mathrm{mg} / \mathrm{kg}$ twice daily, unbound concentrations in the vitreous were predicted to be above the unbound $\mathrm{IC}_{50}$ for only $17 \%$ of the time, and at lower dosages the concentration of unbound nutlin-3a never reached the unbound $\mathrm{IC}_{50}$ level. Simulated concentration-time plots of unbound nutlin-3a after various dosing regimens are also shown in the adrenal gland for neuroblastoma (Figure 4-10), muscle for rhabdomyosarcoma (Figure 4-11) and plasma, bone marrow, and spleen for leukemia (Figure 4-12). The percent times above the unbound $\mathrm{IC}_{50}$ are also listed in (Table 4-4).

\subsection{Discussion}

Nutlin-3a is undergoing preclinical studies examining its potential efficacy for the treatment of several childhood malignancies. Nutlin-3a interrupts the p53-MDM2 protein-protein interaction, which may lead to apoptosis or cell cycle arrest. Treatment of cells with nutlin-3a leads to reversal of multi-drug resistance [228], reduced cell migration [229], reduced angiogenesis [214, 229], radiosensitization of hypoxic cancer cells [29], and inhibition of tumor adaptation to hypoxia [257]. In this study, we developed a mouse PBPK model of nutlin-3a in plasma and multiple tissues of therapeutic interest. This is the first study to provide comprehensive pharmacokinetic data of nutlin-3a in any species. The design of our study included both oral and intravenous dosing at several dosage levels. This permitted the development of a robust model that accurately describes the disposition of nutlin-3a over a wide range of concentrations. The PBPK model was used to design rationale dosing regimens for preclinical models of several malignancies based upon achieving adequate cytotoxic nutlin-3a concentrations within the target organ. This approach is superior to optimizing dosing based solely on plasma concentrations since drug penetration to different organs can vary widely. 

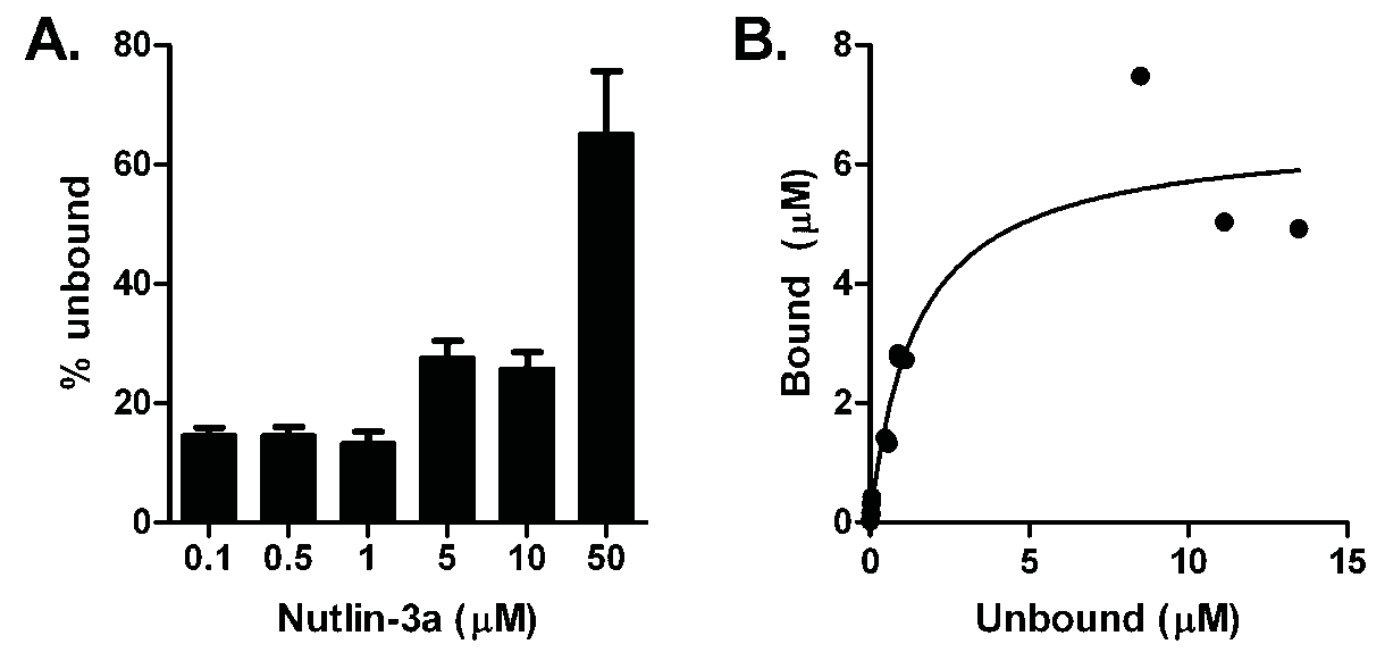

Figure 4-8. Nutlin-3a binding to cell culture media

Bound and unbound nutlin-3a plasma concentrations were determined with equilibrium dialysis.

A. The unbound fraction is shown with increasing total nutlin-3a concentrations. Bars represent the mean and error bars represent the standard deviation for one experiment performed in triplicate.

B. Nonlinear regression was used to fit a binding model (Langmuir equation) to the data.

Table 4-3. Nutlin-3a $\mathrm{IC}_{50}$ for cell survival in different cell types

\begin{tabular}{|c|c|c|c|c|}
\hline Cell type & $\begin{array}{l}\mathrm{IC}_{50} \\
(\mu \mathrm{M})\end{array}$ & $\begin{array}{c}\text { Unbound } \\
\mathrm{IC}_{50} \\
(\mu \mathrm{M})\end{array}$ & $\begin{array}{c}\text { Exposure } \\
\text { time (h) }\end{array}$ & Reference \\
\hline Weril retinoblastoma & 1.1 & 0.21 & 72 & Unpublished $^{\mathrm{a}}$ \\
\hline IMR-32 neuroblastoma & 3.02 & 0.77 & 72 & {$[221]$} \\
\hline RMS-YM rhabodmyosarcoma & $1.25^{\mathrm{b}}$ & 0.26 & 48 & [249] \\
\hline $\begin{array}{c}\text { Primary MDM2-overexpressing } \\
\text { ALL }\end{array}$ & $1.0^{\mathrm{b}}$ & 0.20 & 44 & {$[26]$} \\
\hline
\end{tabular}

a. Mike Dyer, St. Jude Children's Research Hospital, Memphis, TN.

b. Nutlin-3a $\mathrm{IC}_{50}$ assumed to be half of racemic nutlin-3 $\mathrm{IC}_{50}$. 


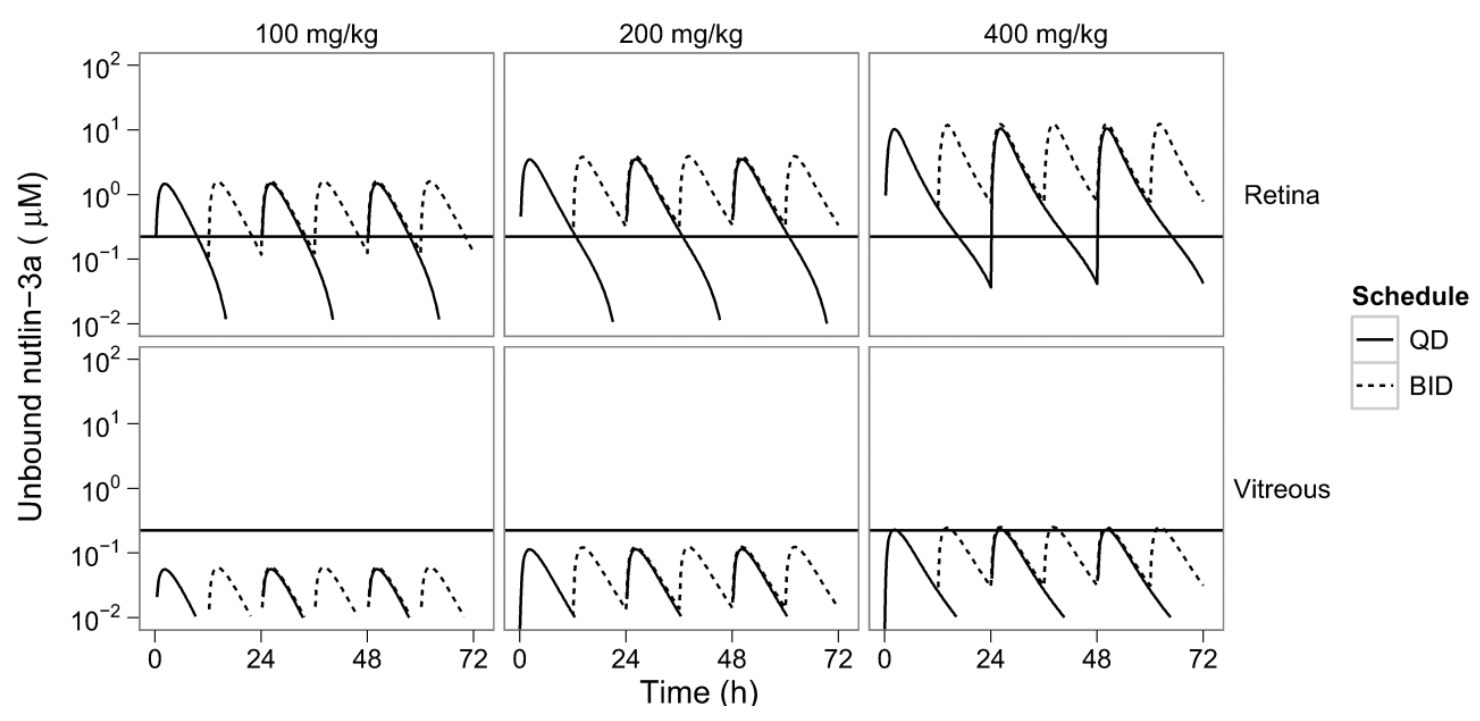

Figure 4-9. Simulated concentration-time plot of unbound nutlin-3a in the retina and vitreous after multiple oral doses given once daily (QD) or twice daily (BID)

The horizontal lines represent the unbound $\mathrm{IC}_{50}$ of nutlin-3a for Weril cells.

Table 4-4. Percent time unbound tissue concentration is above unbound $\mathrm{IC}_{50}$

\begin{tabular}{|c|c|c|c|c|c|c|c|}
\hline \multirow[t]{3}{*}{ Cell line } & \multirow[t]{3}{*}{ Tissue } & \multicolumn{6}{|c|}{ Time above $\mathrm{IC}_{50}(\%)$} \\
\hline & & \multicolumn{3}{|c|}{ QD } & \multicolumn{3}{|c|}{ BID } \\
\hline & & 100 & 200 & 400 & 100 & 200 & 400 \\
\hline \multirow[t]{2}{*}{ Weril } & Retina & 38 & 53 & 70 & 83 & 100 & 100 \\
\hline & Vitreous & 0 & 0 & 5 & 0 & 0 & 17 \\
\hline IMR32 & Adrenal & 27 & 39 & 54 & 58 & 85 & 100 \\
\hline RMS-YM & Muscle & 43 & 58 & 76 & 92 & 100 & 100 \\
\hline \multirow[t]{3}{*}{ ALL } & Plasma & 43 & 57 & 75 & 90 & 100 & 100 \\
\hline & Spleen & 46 & 61 & 80 & 90 & 100 & 100 \\
\hline & Marrow & 0 & 23 & 35 & 12 & 48 & 77 \\
\hline
\end{tabular}

QD: once daily dosing. BID: twice daily dosing. 


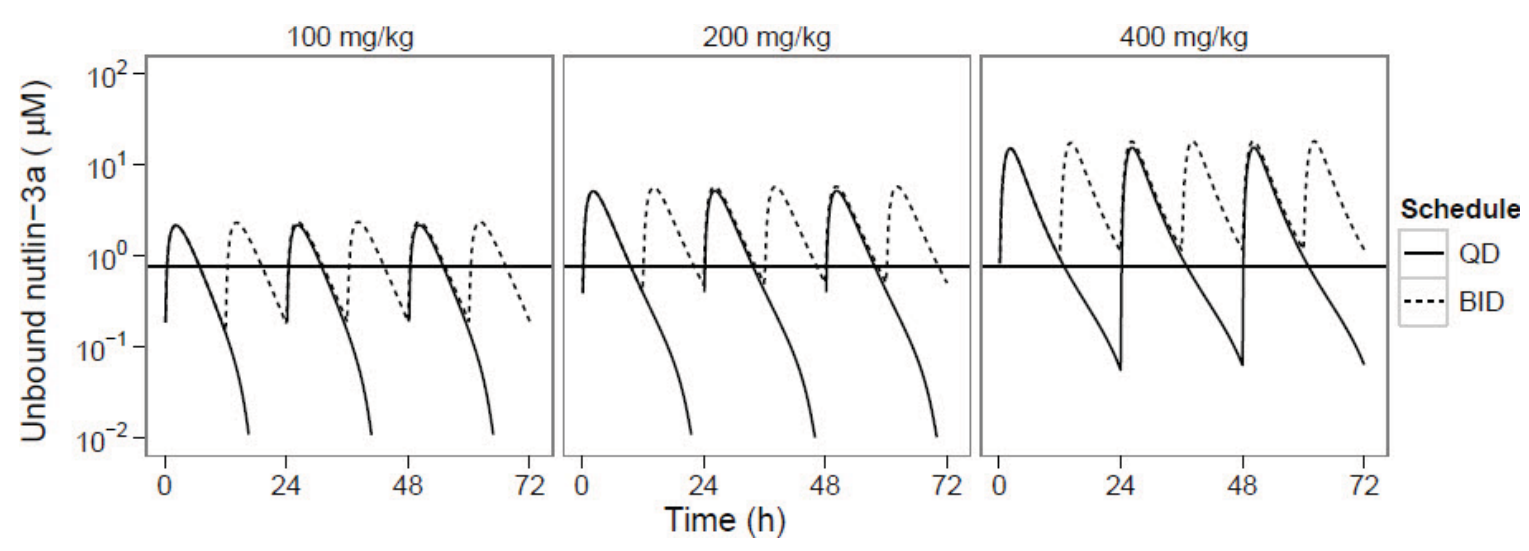

Figure 4-10. Simulated concentration-time plot of unbound nutlin-3a in the adrenal gland after multiple oral doses given once daily (QD) or twice daily (BID)

The horizontal lines represent the unbound $\mathrm{IC}_{50}$ of nutlin-3a for IMR-32 p53-wt neuroblastoma cells.

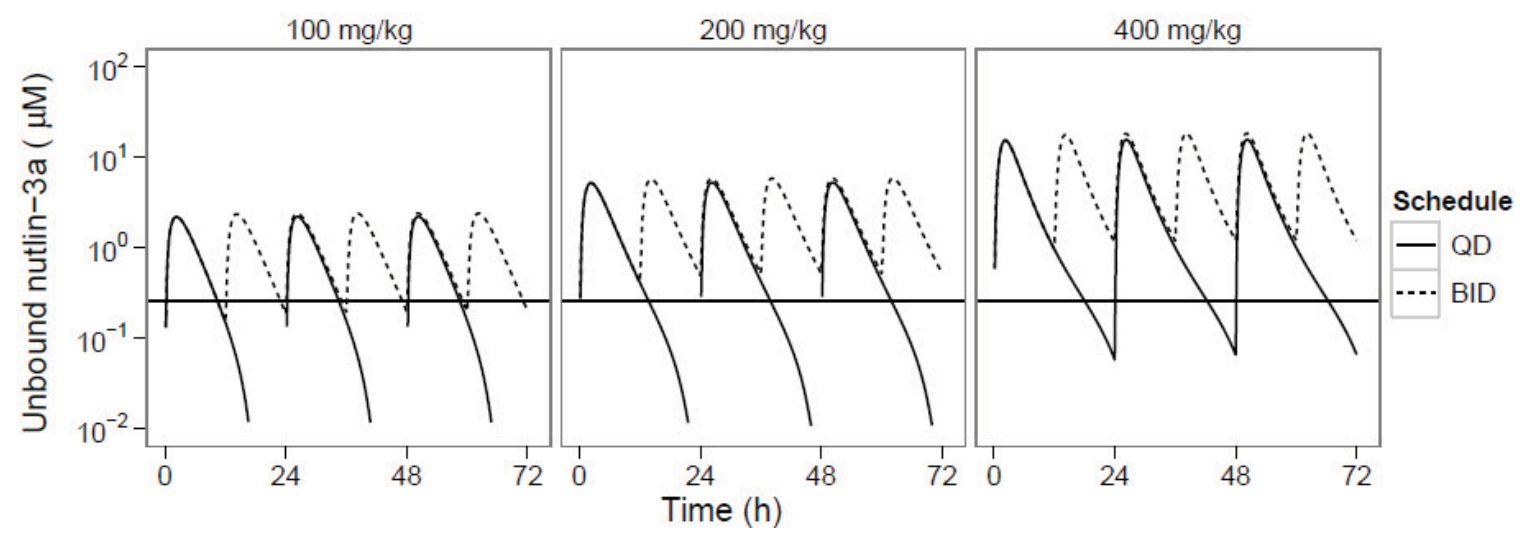

Figure 4-11. Simulated concentration-time plot of unbound nutlin-3a in the muscle after multiple oral doses given once daily (QD) or twice daily (BID)

The horizontal lines represent the unbound $\mathrm{IC}_{50}$ of nutlin-3a for RMS-YM rhabdomyosarcoma cells. 


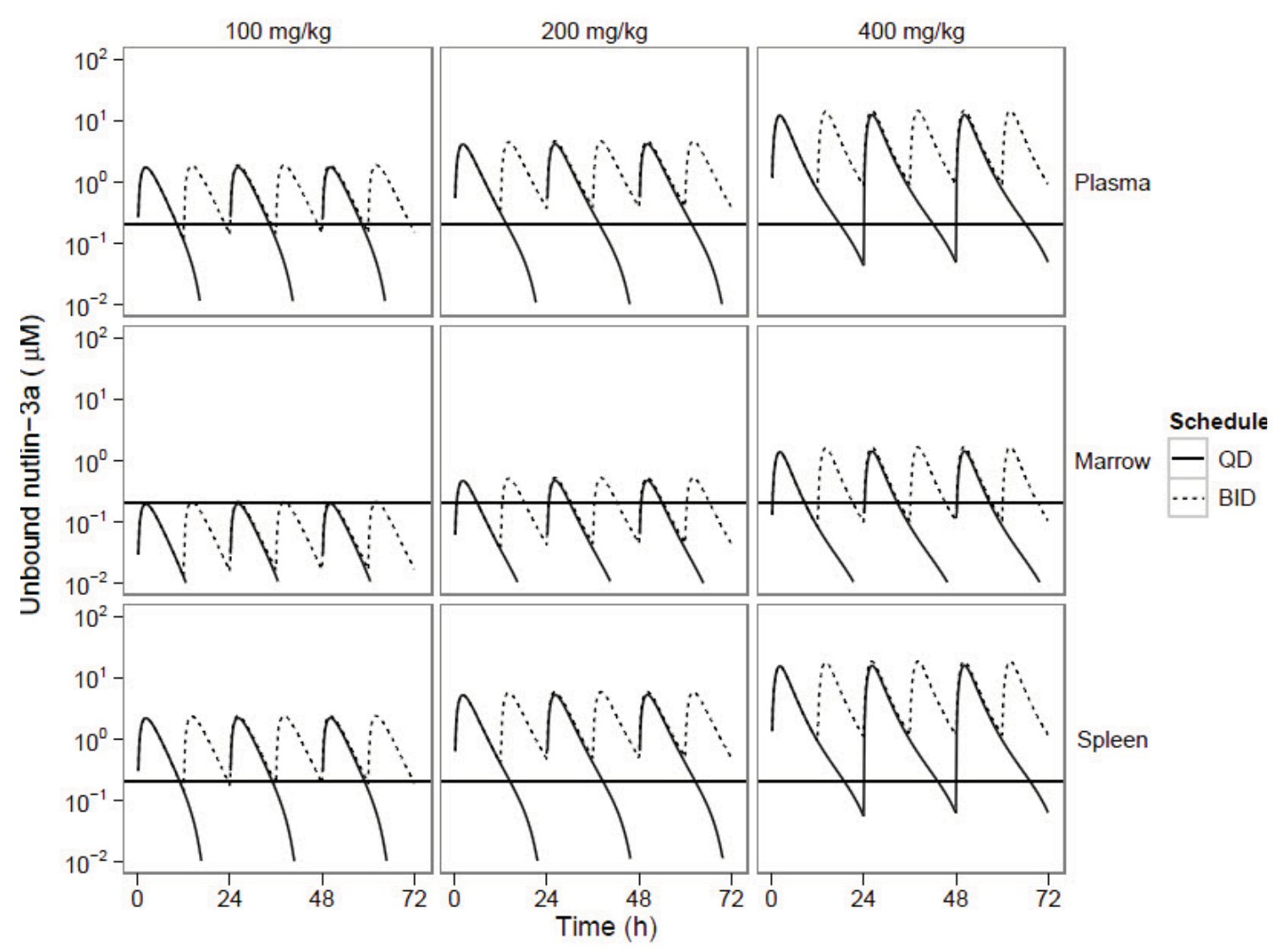

Figure 4-12. Simulated concentration-time plot of unbound nutlin-3a in the plasma, bone marrow, and spleen after multiple oral doses given once daily (QD) or twice daily (BID)

The horizontal lines represent the unbound $\mathrm{IC}_{50}$ of nutlin-3a for MDM2-overexpressing primary acute lymphoblastic leukemia cells. 
Our model describes the key pharmacokinetic properties of nutlin-3a in plasma: rapid absorption, high bioavailability, and saturable elimination that is very rapid at concentrations below $10 \mu \mathrm{M}$. Standard non-compartmental calculations of bioavailability of nutlin-3a were greater than $100 \%$ due to saturable elimination and different ranges of intravenous (10-20 mg/ $\mathrm{kg}$ ) and oral (50-100 mg/ $\mathrm{kg}$ ) dosages. Using the model to simulate the concentration-time profiles after the same dosage administered both intravenous and oral, we were able to estimate nutlin-3a oral bioavailability. Although we performed simulations after multiple doses, the model was based on data from only single doses of nutlin-3a, and therefore should be interpreted with caution.

Nutlin-3a is a substrate for the ATP binding cassette (ABC) transporter P-glycoprotein (P-gp), but at higher concentrations can also inhibit P-gp efflux activity [228]. The inhibition of P-gp function may explain why nutlin-3a is capable of rapid absorption and high bioavailability despite being a P-gp substrate. It is also possible that inhibition of P-gp function underlies saturable nutlin-3a elimination, since P-gp can excrete drugs into both the bile and urine [47]. It is unknown whether nutlin-3a may also inhibit its own metabolism at higher concentrations.

The partition coefficients showed a greater than 1000-fold difference between the tissues with the lowest and highest penetration. The liver and intestine showed the highest penetration. High penetration to the liver could be due to uptake transporters expressed at the hepatocyte membranes, which cause intracellular accumulation of nutlin-3a. The intestine had an atypical profile, possibly due to biliary excretion of nutlin-3a. The blood flow-limited model did not fit well to the intestinal concentration data, limiting the ability to accurately estimate the partition coefficient for this organ.

Retinoblastoma is a tumor of the eye for which a number of orthotopic xenograft and genetic murine models have been developed [258]. Daily subconjunctival administration of nutlin-3a for 5 days was effective in a model of retinoblastoma and when combined with topotecan, an 82-fold reduction in tumor burden with no systemic or ocular side-effects was observed [24]. Our PBPK model shows that with the nutlin-3a regimen most commonly used in preclinical studies $(200 \mathrm{mg} / \mathrm{kg}$ administered orally twice daily), unbound concentrations of nutlin-3a in the retina continuously exceeded the unbound $\mathrm{IC}_{50}$. However, due to poor penetration, the unbound $\mathrm{IC}_{50}$ was never achieved in the vitreous with this regimen and was achieved only transiently at $400 \mathrm{mg} / \mathrm{kg}$ twice daily, suggesting that subconjunctival dosing would be more appropriate for targeting retinoblastoma vitreous seeds $[259,260]$. While the PBPK model cannot predict nutlin-3a pharmacokinetics after subconjunctival administration because the ocular absorption is not known, data from a limited experiment could be combined with the PBPK model to predict exposures in various tissues after ocular administration.

At diagnosis, 98\% of neuroblastoma tumors contain wild-type p53 [209-211], and thus these patients are likely to benefit from reactivation of this pathway. Sensitivity to nutlin-3a has been shown in multiple p53 wild-type neuroblastoma cell lines [221, 228]. Treatment of a subcutaneous UKF-NB- ${ }^{3} \mathrm{rDOX}^{20}$ xenograft model with twice-daily oral nutlin-3a $(200 \mathrm{mg} / \mathrm{kg})$ only partially inhibited tumor growth [43], despite good sensitivity 
$\left(\mathrm{IC}_{50}\right.$ of $\left.5.56 \mu \mathrm{M}\right)$ of these cells in vitro [228]. Because the racemic nutlin-3 mixture was used in this experiment, the assumed equivalent dose would be $100 \mathrm{mg} / \mathrm{kg}$ nutlin-3a twice daily. If unbound plasma concentrations are evaluated (since this was not an orthotopic xenograft), unbound nutlin-3a concentrations are predicted to be continually below the established $\mathrm{IC}_{50}$ with this regimen. However, the pharmacokinetic properties of the racemic mixture are unknown, and it is possible that nutlin-3b influences the saturable elimination or plasma protein binding of nutlin-3a. For a subcutaneous or orthotopic xenograft with similar cell sensitivity, $400 \mathrm{mg} / \mathrm{kg}$ oral nutlin-3a twice daily may result in better activity, since concentrations would continuously be above the $\mathrm{IC}_{50}$. Although the literature and our unpublished observations suggest that twice daily $200 \mathrm{mg} / \mathrm{kg}$ oral dosing is well tolerated in mice [43], further toxicity studies will need to be performed to determine whether higher dosages are tolerable.

Nutlin-3a has also demonstrated cytotoxicity in rhabdomyosarcoma cell lines [228, 249], although it has not yet been tested in a preclinical model of rhabdomyosarcoma. Our model predictions show that the standard twice-daily oral 200 $\mathrm{mg} / \mathrm{kg}$ nutlin-3a regime sufficient to achieve unbound muscle nutlin-3a concentrations that were continuously above the $\mathrm{IC}_{50}$ for the RMS-YM cell line. Nutlin-3a concentrations above the $\mathrm{IC}_{50}$ for primary T-ALL cells $[26,41]$ was achieved in the plasma and spleen with a twice-daily regimen of $100 \mathrm{mg} / \mathrm{kg}$ oral nutlin-3a. However, penetration to the bone marrow was poor, and in order to target this compartment, 400 $\mathrm{mg} / \mathrm{kg}$ twice-daily is recommended, which is predicted to result in unbound nutlin-3a concentrations that are above the $\mathrm{IC}_{50} 77 \%$ percent of the time.

Although the model has a number of applications, it also has limitations. First, we did not perform experiments to determine the route of elimination of nutlin-3a. Preliminary unpublished observations indicate that nutlin-3a is metabolized by mouse liver microsomes, but a model with elimination only from the liver did not adequately fit the nutlin-3a plasma concentration-time data. Not modeling the elimination mechanistically could limit the ability to extrapolate the PBPK model to other species. Second, we performed all experiments in non-tumor bearing mice. Compared to normal tissues, the altered environment in tumors (e.g. vasculature, $\mathrm{pH}$, interstitial fluid pressure) may lead to different local disposition. Although it was not feasible to perform pharmacokinetic studies in multiple preclinical models, data obtained from studies in tumor-bearing mice could be easily incorporated into this PBPK model. Another limitation is the assumption that the unbound fraction of nutlin-3a in tissues was equivalent to the unbound fraction in plasma. We did directly measure nutlin-3a binding in vitreous fluid, which is mostly water, but has a variety of proteins [261]. We also performed simulations at dosages beyond those used to develop the model (i.e. 400 $\mathrm{mg} / \mathrm{kg}$ ). Although we were able to characterize the non-linear elimination at higher plasma concentrations, it is possible that there are unknown non-linear absorption or elimination processes occurring at this higher dosage which would make these model predictions inaccurate.

In summary, we performed extensive mouse pharmacokinetic studies of nutlin-3a and developed a PBPK model, which was utilized to design nutlin-3a dosing regimens for 
preclinical models of pediatric malignancies. Although there are limitations to extrapolating in vitro cytotoxicity data, this analysis provides a starting point for further pharmacokinetic/pharmacodynamic studies in tumor bearing mice. For models of retinoblastoma, the disposition of nutlin-3a after subconjunctival administration should be explored. 


\section{CHAPTER 5. SUMMARY AND FUTURE DIRECTIONS}

The development of more effective and less toxic anti-cancer agents is a slow, high-risk, high-cost, and often high failure process. Potential new agents are emerging continuously from high throughput screening, however, only a small percentage of candidate molecules make their way from bench to bedside. Studies have shown that the success rate for the development of oncology drugs is much lower than that for drugs in other therapeutic areas [262]. In the last few years, the total capitalized cost per approved new drug molecule has risen to $\$ 0.8-1.3$ billion [263-265]. For pediatric malignancies such as retinoblastoma, the small patient pool further hinders the drug development process because of the inability to conduct the clinical trials necessary to evaluate the different compounds. These difficulties necessitate better translational research, where preclinical studies can play an important role.

Since oncology drugs are usually administered in combination, it is important to evaluate the interaction of drugs. Combining agents that act synergistically instead of antagonistically to inhibit cancer cell growth can have a positive impact on patient outcome, taking into consideration that oncology drugs have narrow therapeutic windows. Here we first reported that nutlin-3a synergistically inhibited neuroblastoma cell growth in combination with topotecan and synergistically inhibited BCRP over-expressing osteosarcoma cell growth in combination with mitoxantrone. Preclinical studies from our lab [212] and Michaelis et al. [228] suggested that nutlin-3a inhibited the function of three major efflux transporters BCRP, P-gp, and MRP1. These studies suggested that further in vivo drug-drug interaction studies should be conducted because nutlin-3a may significantly increase the systemic concentrations of agents that are substrates of these transporters. This may cause severe toxicity in clinic thus extra cautious are needed. We still do not know if nutlin-3a also inhibits other efflux or uptake transporters. In addition, because of the interaction of transporters and drug metabolizing enzymes, it is also possible that nutlin-3a may inhibit drug metabolizing enzymes. In fact, our preliminary study suggested that nutlin-3a may be an inhibitor of CYP3A4 (Figure 5-1). Thus, additional preclinical studies are needed to address the interaction of nutlin-3a with CYP3A4 substrates in vitro and in vivo.

Reasons for attrition of oncology new chemical entities during clinical trials include incorrect hypothesis regarding the drug action on the disease, improper preclinical tumor model, improper clinical trial design, and drug toxicity. One additional explanation for this lack of effectiveness in translating laboratory science into efficient therapies is the scarce information on drug concentration within human tumor. The drug exposure at the target site, which is associated with efficacy, can be very different from the plasma exposure. Moreover, different tissues are likely to have different drug penetration characteristics. However, due to ethical and practical reasons, most current clinical trials do not measure drug concentrations directly in tumor or target tissues and instead rely on indirect assessment of drug exposure by measuring drug concentrations in surrogate compartments such as plasma. Thus, if one could develop an approach to reliably estimate human tumor or target tissue drug concentrations from preclinical data, 


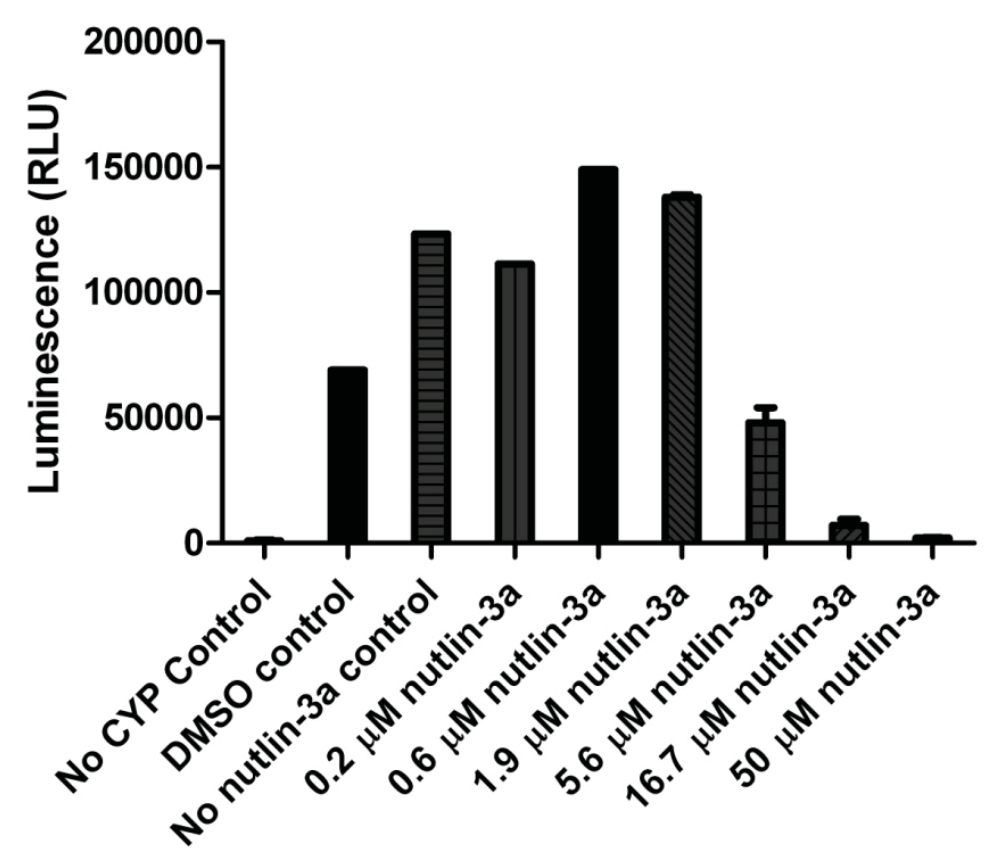

Figure 5-1. Nutlin-3a inhibits the activity of CYP3A4 in vitro

Study was performed using Promega p450glo CYP3A4/Luciferin-IPA Assay (Madison, WI) following manufacture instructions. High luminescence reading represents high CYP3A4 activity. 
the use of drugs could be greatly improved. PK modeling and simulation is an example of such an approach. Once a preclinical model describing both plasma and target tissue/tumor concentration has been established, it can be scaled-up to humans to predict if the dosage necessary for a desirable target tissue or tumor concentration is practically achievable. If the answer is no, a "No-Go" decision should be considered to avoid trial failure and thus save precious resources. When the human plasma PK parameters are generated after either Phase 0 microdosing or after the Phase I clinical trial, the human plasma PK model can be updated to give more accurate prediction of human tissue/tumor concentration. This will allow the clinician to select more "effective" dosages, which are based upon exposures necessary for tumor effect.

In this study, we conducted developed a mouse PBPK model of nutlin-3a in the plasma and multiple tissues of therapeutic interest. This is the first study to provide comprehensive pharmacokinetic data of nutlin-3a in any species. The design of our study included both oral and intravenous dosing at several dosage levels. This method permitted the development of a robust model that accurately describes the disposition of nutlin-3a in the plasma and organs over a wide range of concentrations. The PBPK model was used to suggest dosing regimens to attain putative cytotoxic nutlin-3a exposures in target tissues for retinoblastoma, neuroblastoma, rhabdomyosarcoma, and leukemia. Once nutlin-3a plasma concentration information in human is available (either by scaling or phase 0/I clinical trial), current PBPK model can be updated to predict nutlin-3a exposure in the human tissues.

In future studies, nutlin-3a concentrations in preclinical models of pediatric tumors can be further assessed. One possible tumor to study is retinoblastoma. Nutlin-3a has shown promising efficacy in preclinical studies of retinoblastoma, and will be an excellent compound to use as a model for further PK-PD studies. Subconjunctival administration of nutlin-3a in combination with subconjunctival topotecan for 5 consecutive days reduced the tumor burden by 82 -fold in rats [24]. Subconjunctival nutlin-3a in combination with systemic topotecan demonstrated significant improvement in outcome in mice bearing orthotopic retinoblastoma tumor [42]. It has been shown that the dosing route of nutlin-3a has a great impact on its ocular distribution. PK studies suggested subconjunctival administration of nutlin-3a increased the vitreous exposure by 2000-fold compared to systemic administration in non-tumor bearing mice [42]. However, the amount or extent of nutlin-3a reaching a retinoblastoma tumor in mouse models is still unknown, and it would not be feasible to obtain that data in patients. Thus, the overall goal of the proposed future study is to develop PK models of nutlin-3a disposition in retinoblastoma tumors after subconjunctival injection and systemic administration in orthotopic retinoblastoma xenograft. Both classical and PBPK model will be developed. Major challenges include determining retinoblastoma tumor blood flow, dealing with large variations of nutlin-3a concentrations in tumor, vitreous, and retina, and the structural complexity of the pharmacokinetic models. Other methodological issues to be addressed include the extremely small volume $(<8 \mu \mathrm{L}$ per sample) of tissue samples (tumor, vitreous and retina), which could be an obstacle for both sample collection and LC-MS/MS analysis. However, when developed, these pharmacokinetic models would be the first to describe drug concentration in 
retinoblastoma tumors with the ultimate goal to translate the findings to the design of clinical trials. 


\section{LIST OF REFERENCES}

1. Brown, C.J., et al., Awakening guardian angels: drugging the p53 pathway. Nature Reviews. Cancer, 2009. 9(12): p. 862-73.

2. Speidel, D., Transcription-independent 553 apoptosis: an alternative route to death. Trends in Cell Biology, 2010. 20(1): p. 14-24.

3. Moll, U.M., et al., Transcription-independent pro-apoptotic functions of p53. Current Opinion in Cell Biology, 2005. 17(6): p. 631-6.

4. Cheok, C.F., et al., Translating p53 into the clinic. Nature Reviews. Clinical Oncology, 2011. 8(1): p. 25-37.

5. Reich, N.C., et al., Two distinct mechanisms regulate the levels of a cellular tumor antigen, p53. Molecular and Cellular Biology, 1983. 3(12): p. 2143-50.

6. Tao, W., et al., Nucleocytoplasmic shuttling of oncoprotein Hdm2 is required for Hdm2-mediated degradation of p53. Proceedings of the National Academy of Sciences of the United States of America, 1999. 96(6): p. 3077-80.

7. Haupt, Y., et al., Mdm2 promotes the rapid degradation of p53. Nature, 1997. 387(6630): p. 296-9.

8. Momand, J., et al., The mdm-2 oncogene product forms a complex with the 553 protein and inhibits p53-mediated transactivation. Cell, 1992. 69(7): p. 1237-45.

9. Bond, G.L., et al., MDM2 is a central node in the 533 pathway: 12 years and counting. Current Cancer Drug Targets, 2005. 5(1): p. 3-8.

10. Marine, J.C., et al., MDMX: from bench to bedside. Journal of Cell Science, 2007. 120(Pt 3): p. 371-8.

11. Finch, R.A., et al., Mdmx is a negative regulator of p53 activity in vivo. Cancer Research, 2002. 62(11): p. 3221-5.

12. Wade, M., et al., The p53 orchestra: Mdm2 and Mdmx set the tone. Trends in Cell Biology, 2010. 20(5): p. 299-309.

13. Linares, L.K., et al., HdmX stimulates Hdm2-mediated ubiquitination and degradation of p53. Proceedings of the National Academy of Sciences of the United States of America, 2003. 100(21): p. 12009-14.

14. Gu, J., et al., Mutual dependence of $M D M 2$ and $M D M X$ in their functional inactivation of p53. The Journal of Biological Chemistry, 2002. 277(22): p. 19251-4. 
15. Chene, P., Inhibiting the p53-MDM2 interaction: an important target for cancer therapy. Nature Reviews. Cancer, 2003. 3(2): p. 102-9.

16. Shangary, S., et al., Temporal activation of p53 by a specific MDM2 inhibitor is selectively toxic to tumors and leads to complete tumor growth inhibition. Proceedings of the National Academy of Sciences of the United States of America, 2008. 105(10): p. 3933-8.

17. Vassilev, L.T., et al., In vivo activation of the 553 pathway by small-molecule antagonists of MDM2. Science, 2004. 303(5659): p. 844-8.

18. Reed, D., et al., Identification and characterization of the first small molecule inhibitor of MDMX. The Journal of Biological Chemistry, 2010. 285(14): p. 10786-96.

19. Grasberger, B.L., et al., Discovery and cocrystal structure of benzodiazepinedione HDM2 antagonists that activate p53 in cells. Journal of Medicinal Chemistry, 2005. 48(4): p. 909-12.

20. Kussie, P.H., et al., Structure of the MDM2 oncoprotein bound to the p53 tumor suppressor transactivation domain. Science, 1996. 274(5289): p. 948-53.

21. Shangary, S., et al., Small-molecule inhibitors of the MDM2-p53 protein-protein interaction to reactivate p53 function: a novel approach for cancer therapy. Annual Review of Pharmacology and Toxicology, 2009. 49: p. 223-41.

22. Vassilev, L.T., p53 Activation by small molecules: application in oncology. Journal of Medicinal Chemistry, 2005. 48(14): p. 4491-9.

23. Bottger, V., et al., Comparative study of the p53-mdm2 and p53-MDMX interfaces. Oncogene, 1999. 18(1): p. 189-99.

24. Laurie, N.A., et al., Inactivation of the p53 pathway in retinoblastoma. Nature, 2006. 444(7115): p. 61-6.

25. Tovar, C., et al., Small-molecule MDM2 antagonists reveal aberrant p53 signaling in cancer: implications for therapy. Proceedings of the National Academy of Sciences of the United States of America, 2006. 103(6): p. 1888-93.

26. Gu, L., et al., MDM2 antagonist nutlin-3 is a potent inducer of apoptosis in pediatric acute lymphoblastic leukemia cells with wild-type p53 and overexpression of MDM2. Leukemia, 2008. 22(4): p. 730-9.

27. $\mathrm{Hu}, \mathrm{B}$., et al., MDMX overexpression prevents $p 53$ activation by the MDM2 inhibitor nutlin. The Journal of Biological Chemistry, 2006. 281(44): p. 33030-5.

28. Kranz, D., et al., Nongenotoxic 553 activation protects cells against $S$-phasespecific chemotherapy. Cancer Research, 2006. 66(21): p. 10274-80. 
29. Supiot, S., et al., Nutlin-3 radiosensitizes hypoxic prostate cancer cells independent of p53. Molecular Cancer Therapeutics, 2008. 7(4): p. 993-9.

30. Carter, B.Z., et al., Simultaneous activation of p53 and inhibition of XIAP enhance the activation of apoptosis signaling pathways in AML. Blood, 2010. 115(2): p. 306-14.

31. Zhang, W., et al., Blockade of mitogen-activated protein kinase/extracellular signal-regulated kinase kinase and murine double minute synergistically induces Apoptosis in acute myeloid leukemia via BH3-only proteins Puma and Bim. Cancer Research, 2010. 70(6): p. 2424-34.

32. Coll-Mulet, L., et al., MDM2 antagonists activate p 53 and synergize with genotoxic drugs in B-cell chronic lymphocytic leukemia cells. Blood, 2006. 107(10): p. 4109-14.

33. Kojima, K., et al., Mdm2 inhibitor Nutlin-3a induces p53-mediated apoptosis by transcription-dependent and transcription-independent mechanisms and may overcome Atm-mediated resistance to fludarabine in chronic lymphocytic leukemia. Blood, 2006. 108(3): p. 993-1000.

34. Secchiero, P., et al., Functional integrity of the p53-mediated apoptotic pathway induced by the nongenotoxic agent nutlin-3 in B-cell chronic lymphocytic leukemia (B-CLL). Blood, 2006. 107(10): p. 4122-9.

35. Zauli, G., et al., Dasatinib plus Nutlin-3 shows synergistic antileukemic activity in both $p 53$ wild-type and $p 53$ mutated B chronic lymphocytic leukemias by inhibiting the Akt pathway. Clinical Cancer Research, 2011. 17(4): p. 762-70.

36. Ribas, J., et al., (R)-roscovitine (CYC202, Seliciclib) sensitizes SH-SY5Y neuroblastoma cells to nutlin-3-induced apoptosis. Experimental Cell Research, 2006. 312(12): p. 2394-400.

37. Saha, M.N., et al., MDM2 antagonist nutlin plus proteasome inhibitor velcade combination displays a synergistic anti-myeloma activity. Cancer Biology \& Therapy, 2010. 9(11): p. 936-44.

38. Ooi, M.G., et al., Interactions of the Hdm2/p53 and proteasome pathways may enhance the antitumor activity of bortezomib. Clinical Cancer Research, 2009. 15(23): p. 7153-60.

39. Vassilev, L.T., Small-molecule antagonists of p53-MDM2 binding: research tools and potential therapeutics. Cell Cycle, 2004. 3(4): p. 419-21.

40. Sarek, G., et al., P53 reactivation kills KSHV lymphomas efficiently in vitro and in vivo: new hope for treating aggressive viral lymphomas. Cell Cycle, 2007. 6(18): p. 2205-9. 
41. Kojima, K., et al., MDM2 antagonists induce p53-dependent apoptosis in AML: implications for leukemia therapy. Blood, 2005. 106(9): p. 3150-9.

42. Brennan, R.C., et al., Targeting the p53 pathway in retinoblastoma with subconjunctival nutlin-3a. Cancer Research, 2011. 71(12): p. 4205-13.

43. Van Maerken, T., et al., Antitumor activity of the selective MDM2 antagonist nutlin-3 against chemoresistant neuroblastoma with wild-type p53. Journal of the National Cancer Institute, 2009. 101(22): p. 1562-74.

44. He, L., et al., Analysis and update of the human solute carrier (SLC) gene superfamily. Human Genomics, 2009. 3(2): p. 195-206.

45. Vasiliou, V., et al., Human ATP-binding cassette (ABC) transporter family. Human Genomics, 2009. 3(3): p. 281-90.

46. Hediger, M.A., et al., The ABCs of solute carriers: physiological, pathological and therapeutic implications of human membrane transport proteins. Pflugers Archiv : European Journal of Physiology, 2004. 447(5): p. 465-8.

47. Giacomini, K.M., et al., Membrane transporters in drug development. Nature Reviews. Drug Discovery, 2010. 9(3): p. 215-36.

48. Rees, D.C., et al., ABC transporters: the power to change. Nature Reviews. Molecular Cell Biology, 2009. 10(3): p. 218-27.

49. Davidson, A.L., et al., ABC transporters: how small machines do a big job. Trends in Microbiology, 2007. 15(10): p. 448-55.

50. Gottesman, M.M., et al., Multidrug resistance in cancer: role of ATP-dependent transporters. Nature Reviews. Cancer, 2002. 2(1): p. 48-58.

51. Hollenstein, K., et al., Structure and mechanism of ABC transporter proteins. Current Opinion in Structural Biology, 2007. 17(4): p. 412-8.

52. Higgins, C.F., ABC transporters: physiology, structure and mechanism--an overview. Research in Microbiology, 2001. 152(3-4): p. 205-10.

53. Robey, R.W., et al., ABCG2: a perspective. Advanced Drug Delivery Reviews, 2009. 61(1): p. 3-13.

54. Biedler, J.L., et al., Cellular resistance to actinomycin D in Chinese hamster cells in vitro: cross-resistance, radioautographic, and cytogenetic studies. Cancer Research, 1970. 30(4): p. 1174-84.

55. Akiyama, S., et al., Isolation and genetic characterization of human KB cell lines resistant to multiple drugs. Somatic Cell and Molecular Genetics, 1985. 11(2): p. 117-26. 
56. Roninson, I.B., et al., Isolation of human mdr DNA sequences amplified in multidrug-resistant KB carcinoma cells. Proceedings of the National Academy of Sciences of the United States of America, 1986. 83(12): p. 4538-42.

57. Miyake, K., et al., Molecular cloning of cDNAs which are highly overexpressed in mitoxantrone-resistant cells: demonstration of homology to ABC transport genes. Cancer Research, 1999. 59(1): p. 8-13.

58. Sarkadi, B., et al., Human multidrug resistance $A B C B$ and $A B C G$ transporters: participation in a chemoimmunity defense system. Physiological Reviews, 2006. 86(4): p. 1179-236.

59. Marquez, B., et al., ABC multidrug transporters: target for modulation of drug pharmacokinetics and drug-drug interactions. Current Drug Targets, 2011. 12(5): p. $600-20$.

60. Sparreboom, A., et al., Limited oral bioavailability and active epithelial excretion of paclitaxel (Taxol) caused by P-glycoprotein in the intestine. Proceedings of the National Academy of Sciences of the United States of America, 1997. 94(5): p. 2031-5.

61. Bardelmeijer, H.A., et al., Low systemic exposure of oral docetaxel in mice resulting from extensive first-pass metabolism is boosted by ritonavir. Cancer Research, 2002. 62(21): p. 6158-64.

62. Leggas, M., et al., Gefitinib modulates the function of multiple ATP-binding cassette transporters in vivo. Cancer Research, 2006. 66(9): p. 4802-7.

63. Beedholm-Ebsen, R., et al., Identification of multidrug resistance protein 1 (MRP1/ABCC1) as a molecular gate for cellular export of cobalamin. Blood, 2010. 115(8): p. 1632-9.

64. Bosquillon, C., Drug transporters in the lung--do they play a role in the biopharmaceutics of inhaled drugs? Journal of Pharmaceutical Sciences, 2010. 99(5): p. 2240-55.

65. Su, L., et al., Drug transporters, the blood-testis barrier, and spermatogenesis. The Journal of Endocrinology, 2011. 208(3): p. 207-23.

66. Mannermaa, E., et al., Drug transport in corneal epithelium and blood-retina barrier: emerging role of transporters in ocular pharmacokinetics. Advanced Drug Delivery Reviews, 2006. 58(11): p. 1136-63.

67. Couture, L., et al., The ATP-binding cassette transporters and their implication in drug disposition: a special look at the heart. Pharmacological Reviews, 2006. 58(2): p. 244-58. 
68. Glavinas, H., et al., The role of ABC transporters in drug resistance, metabolism and toxicity. Current Drug Delivery, 2004. 1(1): p. 27-42.

69. Polli, J.W., et al., Rational use of in vitro P-glycoprotein assays in drug discovery. The Journal of Pharmacology and Experimental Therapeutics, 2001. 299(2): p. $620-8$.

70. Gupta, A., et al., HIV protease inhibitors are inhibitors but not substrates of the human breast cancer resistance protein (BCRP/ABCG2). The Journal of Pharmacology and Experimental Therapeutics, 2004. 310(1): p. 334-41.

71. Polli, J.W., et al., Role of P-glycoprotein on the CNS disposition of amprenavir (141W94), an HIV protease inhibitor. Pharmaceutical Research, 1999. 16(8): p. 1206-12.

72. Lee, C.A., et al., P-glycoprotein related drug interactions: clinical importance and a consideration of disease states. Expert Opinion on Drug Metabolism \& Toxicology, 2010. 6(5): p. 603-19.

73. Salphati, L., Transport-metabolism interplay. Molecular Pharmaceutics, 2009. 6(6): p. 1629-30.

74. Krishna, R., et al., Multidrug resistance (MDR) in cancer. Mechanisms, reversal using modulators of MDR and the role of MDR modulators in influencing the pharmacokinetics of anticancer drugs. European Journal of Pharmaceutical Sciences, 2000. 11(4): p. 265-83.

75. Mellor, H.R., et al., Resistance to chemotherapy in cancer: a complex and integrated cellular response. Pharmacology, 2008. 81(4): p. 275-300.

76. Baguley, B.C., Multiple drug resistance mechanisms in cancer. Molecular Biotechnology, 2010. 46(3): p. 308-16.

77. Gillet, J.P., et al., Chemotherapy-induced resistance by ATP-binding cassette transporter genes. Biochimica et Biophysica Acta, 2007. 1775(2): p. 237-62.

78. Bakos, E., et al., Portrait of multifaceted transporter, the multidrug resistanceassociated protein 1 (MRP1/ABCC1). Pflugers Archiv : European Journal of Physiology, 2007. 453(5): p. 621-41.

79. Lemos, C., et al., Drug transporters: recent advances concerning BCRP and tyrosine kinase inhibitors. British Journal of Cancer, 2008. 98(5): p. 857-62.

80. Szakács, G., et al., Multidrug resistance mediated by MDR-ABC transporters. In: Siddik, Z.H., et al., editors. Drug Resistance in Cancer Cells. New York: Springers; 2009. p. 1-20. 
81. O'Connor, R., The pharmacology of cancer resistance. Anticancer Research, 2007. 27(3A): p. 1267-72.

82. Ross, D.D., et al. Impact of breast cancer resistance protein on cancer treatment outcomes. In: Zhou, J., editor. Multi-Drug Resistance in Cancer. New York: Humana Press; 2010. p.251-90.

83. Raguz, S., et al., Resistance to chemotherapy: new treatments and novel insights into an old problem. British Journal of Cancer, 2008. 99(3): p. 387-91.

84. Ischenko, I., et al., Cancer stem cells: how can we target them? Current Medicinal Chemistry 2008. 15(30): p. 3171-84.

85. Oldham, M.L., et al., Structural insights into ABC transporter mechanism. Current Opinion in Structural Biology, 2008. 18(6): p. 726-33.

86. Aller, S.G., et al., Structure of P-glycoprotein reveals a molecular basis for polyspecific drug binding. Science, 2009. 323(5922): p. 1718-22.

87. Gutmann, D.A., et al., Understanding polyspecificity of multidrug $A B C$ transporters: closing in on the gaps in $A B C B 1$. Trends in Biochemical Sciences, 2010. 35(1): p. 36-42.

88. Hennessy, M., et al., A primer on the mechanics of P-glycoprotein the multidrug transporter. Pharmacological Research : the Official Journal of the Italian Pharmacological Society, 2007. 55(1): p. 1-15.

89. Ramachandra, M., et al., Human P-glycoprotein exhibits reduced affinity for substrates during a catalytic transition state. Biochemistry, 1998. 37(14): p. 5010-9.

90. Procko, E., et al., The mechanism of ABC transporters: general lessons from structural and functional studies of an antigenic peptide transporter. The FASEB Journal, 2009. 23(5): p. 1287-302.

91. Sauna, Z.E., et al., Evidence for a requirement for ATP hydrolysis at two distinct steps during a single turnover of the catalytic cycle of human P-glycoprotein. Proceedings of the National Academy of Sciences of the United States of America, 2000. 97(6): p. 2515-20.

92. Kage, K., et al., Dominant-negative inhibition of breast cancer resistance protein as drug efflux pump through the inhibition of $S$-S dependent homodimerization. International Journal of Cancer, 2002. 97(5): p. 626-30.

93. Sarkadi, B., et al., Expression of the human multidrug resistance cDNA in insect cells generates a high activity drug-stimulated membrane ATPase. The Journal of Biological Chemistry 1992. 267(7): p. 4854-8. 
94. Glavinas, H., et al., ABCG2 (breast cancer resistance protein/mitoxantrone resistance-associated protein) ATPase assay: a useful tool to detect drugtransporter interactions. Drug Metabolism and Disposition, 2007. 35(9): p. 153342.

95. Bush, J.A., et al., Cancer chemoresistance: the relationship between $p 53$ and multidrug transporters. International Journal of Cancer, 2002. 98(3): p. 323-30.

96. Bahr, O., et al., Modulation of MDR/MRP by wild-type and mutant $p 53$. The Journal of Clinical Investigation, 2001. 107(5): p. 643-6.

97. Chin, K.V., et al., Modulation of activity of the promoter of the human MDRI gene by Ras and p53. Science, 1992. 255(5043): p. 459-62.

98. Strauss, B.E., et al., The MDR1 downstream promoter contains sequence-specific binding sites for wild-type p53. Biochemical and Biophysical Research Communications, 1995. 217(3): p. 825-31.

99. Zastawny, R.L., et al., The core promoter region of the P-glycoprotein gene is sufficient to confer differential responsiveness to wild-type and mutant p53. Oncogene, 1993. 8(6): p. 1529-35.

100. Nguyen, K.T., et al., Transactivation of the human multidrug resistance (MDR1) gene promoter by p53 mutants. Oncology Research, 1994. 6(2): p. 71-7.

101. Sampath, J., et al., Mutant $p 53$ cooperates with ETS and selectively up-regulates human MDR1 not MRP1. The Journal of Biological Chemistry, 2001. 276(42): p. 39359-67.

102. Thottassery, J.V., et al., P53-dependent regulation of MDR1 gene expression causes selective resistance to chemotherapeutic agents. Proceedings of the National Academy of Sciences of the United States of America, 1997. 94(20): p. 11037-42.

103. Goldsmith, M.E., et al., Wild type p53 stimulates expression from the human multidrug resistance promoter in a p53-negative cell line. The Journal of Biological Chemistry, 1995. 270(4): p. 1894-8.

104. Kopnin, B.P., et al., Influence of exogenous ras and 53 on P-glycoprotein function in immortalized rodent fibroblasts. Oncology Research, 1995. 7(6): p. 299-306.

105. Nicoletti, M.I., et al., Wild-type 553 marginally induces endogenous MDR-1 $m R N A$ without causing a measurable drug resistance in human cancer cells. International Journal of Oncology, 2001. 18(2): p. 375-81. 
106. Wang, Q., et al., Transcriptional suppression of multidrug resistance-associated protein (MRP) gene expression by wild-type p53. Cancer Research, 1998. 58(24): p. 5762-9.

107. Wang, X., et al., Transcriptional suppression of breast cancer resistance protein (BCRP) by wild-type 533 through the NF-kappaB pathway in MCF-7 cells. FEBS Letters, 2010. 584(15): p. 3392-7.

108. Rengelshausen, J., et al., Contribution of increased oral bioavailability and reduced nonglomerular renal clearance of digoxin to the digoxin-clarithromycin interaction. British Journal of Clinical Pharmacology, 2003. 56(1): p. 32-8.

109. Sun, H., et al., The Caco-2 cell monolayer: usefulness and limitations. Expert Opinion on Drug Metabolism \& Toxicology, 2008. 4(4): p. 395-411.

110. Hayeshi, R., et al., Comparison of drug transporter gene expression and functionality in Caco-2 cells from 10 different laboratories. European Journal of Pharmaceutical Sciences, 2008. 35(5): p. 383-96.

111. Kuteykin-Teplyakov, K., et al., Differences in the expression of endogenous efflux transporters in MDR1-transfected versus wildtype cell lines affect P-glycoprotein mediated drug transport. British Journal of Pharmacology, 2010. 160(6): p. 145363.

112. Di, L., et al., Development of a new permeability assay using low-efflux MDCKII cells. Journal of Pharmaceutical Sciences, 2011. 100(11): p. 4974-85.

113. Goh, L.B., et al., Endogenous drug transporters in in vitro and in vivo models for the prediction of drug disposition in man. Biochemical Pharmacology, 2002. 64(11): p. 1569-78.

114. Zhang, L., et al., A regulatory viewpoint on transporter-based drug interactions. Xenobiotica, 2008. 38(7-8): p. 709-24.

115. Kawai, R., et al., Physiologically based pharmacokinetics of cyclosporine A: extension to tissue distribution kinetics in rats and scale-up to human. The Journal of Pharmacology and Experimental Therapeutics, 1998. 287(2): p. 45768.

116. Nestorov, I., Whole body pharmacokinetic models. Clinical Pharmacokinetics, 2003. 42(10): p. 883-908.

117. Nestorov, I., Whole-body physiologically based pharmacokinetic models. Expert Opinion on Drug Metabolism \& Toxicology, 2007. 3(2): p. 235-49.

118. Aarons, L., Physiologically based pharmacokinetic modelling: a sound mechanistic basis is needed. British Journal of Clinical Pharmacology, 2005. 60(6): p. 581-3. 
119. Rowland, M., et al., Physiologically-based pharmacokinetics in drug development and regulatory science. Annual Review of Pharmacology and Toxicology, 2011. 51: p. 45-73.

120. Edginton, A.N., et al., Whole body physiologically-based pharmacokinetic models: their use in clinical drug development. Expert Opinion on Drug Metabolism \& Toxicology, 2008. 4(9): p. 1143-52.

121. Poulin, P., et al., PhRMA CPCDC initiative on predictive models of human pharmacokinetics, part 1: Goals, properties of the PhRMA dataset, and comparison with literature datasets. Journal of Pharmaceutical Sciences, 2011. Accessed Sep 6, 2011, Available from: http://dx.doi.org/10.1002/jps.22554.

122. Jones, R.D., et al., PhRMA CPCDC initiative on predictive models of human pharmacokinetics, part 2: Comparative assessment of prediction methods of human volume of distribution. Journal of Pharmaceutical Sciences, 2011. Accessed Sep 6, 2011, Available from: http://dx.doi.org/10.1002/jps.22553.

123. Ring, B.J., et al., PhRMA CPCDC initiative on predictive models of human pharmacokinetics, part 3: Comparative assessement of prediction methods of human clearance. Journal of Pharmaceutical Sciences, 2011. Accessed Sep 6, 2011, Available from: http://dx.doi.org/10.1002/jps.22552.

124. Vuppugalla, R., et al., PhRMA CPCDC initiative on predictive models of human pharmacokinetics, part 4: Prediction of plasma concentration-time profiles in human from in vivo preclinical data by using the Wajima approach. Journal of Pharmaceutical Sciences, 2011. Accessed Sep 6, 2011, Available from: http:// dx.doi.org/10.1002/jps.22551.

125. Poulin, P., et al., PhRMA CPCDC initiative on predictive models of human pharmacokinetics, part 5: Prediction of plasma concentration-time profiles in human by using the physiologically-based pharmacokinetic modeling approach. Journal of Pharmaceutical Sciences, 2011. Accessed Sep 6, 2011, Available from: http://dx.doi.org/10.1002/jps.22550.

126. Bjorkman, S., Prediction of drug disposition in infants and children by means of physiologically based pharmacokinetic (PBPK) modelling: theophylline and midazolam as model drugs. British Journal of Clinical Pharmacology, 2005. 59(6): p. 691-704.

127. Johnson, T.N., et al., Prediction of the clearance of eleven drugs and associated variability in neonates, infants and children. Clinical Pharmacokinetics, 2006. 45(9): p. 931-56.

128. Kersting, G., et al., Physiologically based pharmacokinetic modelling of highand low-dose etoposide: from adults to children. Cancer Chemotherapy and Pharmacology, 2011. Accessed Sep 6, 2011, Available from: http://dx.doi.org/ 10.1007/s00280-011-1706-9. 
129. Edginton, A.N., Knowledge-driven approaches for the guidance of first-inchildren dosing. Paediatric Anaesthesia, 2011. 21(3): p. 206-13.

130. Johnson, T.N., et al., Resurgence in the use of physiologically based pharmacokinetic models in pediatric clinical pharmacology: parallel shift in incorporating the knowledge of biological elements and increased applicability to drug development and clinical practice. Paediatric Anaesthesia, 2011. 21(3): p. 291-301.

131. Khalil, F., et al., Physiologically based pharmacokinetic modeling: methodology, applications, and limitations with a focus on its role in pediatric drug development. Journal of Biomedicine \& Biotechnology, 2011. 2011: p. 907461.

132. Yang, F., et al., Population-based analysis of methadone distribution and metabolism using an age-dependent physiologically based pharmacokinetic model. Journal of Pharmacokinetics and Pharmacodynamics, 2006. 33(4): p. 485518.

133. Ginsberg, G., et al., Physiologically based pharmacokinetic (PBPK) modeling of caffeine and theophylline in neonates and adults: implications for assessing children's risks from environmental agents. Journal of Toxicology and Environmental Health. Part A, 2004. 67(4): p. 297-329.

134. Parrott, N., et al., Development of a physiologically based model for oseltamivir and simulation of pharmacokinetics in neonates and infants. Clinical Pharmacokinetics, 2011. 50(9): p. 613-23.

135. Bjorkman, S., et al., Prediction of the disposition of midazolam in surgical patients by a physiologically based pharmacokinetic model. Journal of Pharmaceutical Sciences, 2001. 90(9): p. 1226-41.

136. Li, J., et al., The effect of age on the early disposition of doxorubicin. Cancer Chemotherapy and Pharmacology, 2003. 51(5): p. 395-402.

137. Hays, S.M., et al., Development of a physiologically based pharmacokinetic model of 2-methoxyethanol and 2-methoxyacetic acid disposition in pregnant rats. Toxicology and Applied Pharmacology, 2000. 163(1): p. 67-74.

138. Gray, D.G., A physiologically based pharmacokinetic model for methyl mercury in the pregnant rat and fetus. Toxicology and Applied Pharmacology, 1995. 132(1): p. 91-102.

139. Terry, K.K., et al., Development of a physiologically based pharmacokinetic model describing 2-methoxyacetic acid disposition in the pregnant mouse. Toxicology and Applied Pharmacology, 1995. 132(1): p. 103-14. 
140. Fisher, J.W., et al., Physiologically based pharmacokinetic modeling of the lactating rat and nursing pup: a multiroute exposure model for trichloroethylene and its metabolite, trichloroacetic acid. Toxicology and Applied Pharmacology, 1990. 102(3): p. 497-513.

141. Yoon, M., et al., Lactational transfer of manganese in rats: predicting manganese tissue concentration in the dam and pups from inhalation exposure with a pharmacokinetic model. Toxicological Sciences, 2009. 112(1): p. 23-43.

142. Edginton, A.N., et al., Physiology-based simulations of a pathological condition: prediction of pharmacokinetics in patients with liver cirrhosis. Clinical Pharmacokinetics, 2008. 47(11): p. 743-52.

143. Haddad, S., et al., Extrapolating in vitro metabolic interactions to isolated perfused liver: predictions of metabolic interactions between R-bufuralol, bunitrolol, and debrisoquine. Journal of Pharmaceutical Sciences, 2010. 99(10): p. 4406-26.

144. Perdaems, N., et al., Predictions of metabolic drug-drug interactions using physiologically based modelling: Two cytochrome P450 3 A4 substrates coadministered with ketoconazole or verapamil. Clinical Pharmacokinetics, 2010. 49(4): p. 239-58.

145. Bois, F.Y., Physiologically based modelling and prediction of drug interactions. Basic \& Clinical Pharmacology \& Toxicology, 2010. 106(3): p. 154-61.

146. Rekic, D., et al., In silico prediction of efavirenz and rifampicin drug-drug interaction considering weight and CYP2B6 phenotype. British Journal of Clinical Pharmacology, 2011. 71(4): p. 536-43.

147. Peck, C.C., Quantitative clinical pharmacology is transforming drug regulation. Journal of Pharmacokinetics and Pharmacodynamics, 2010. 37(6): p. 617-28.

148. Ito, K., et al., Use of clearance concepts and modeling techniques in the prediction of metabolic drug-drug interactions. Trends in Pharmacological Sciences, 2010. 31(8): p. 351-5.

149. Zhao, P., et al., Applications of physiologically based pharmacokinetic (PBPK) modeling and simulation during regulatory review. Clinical Pharmacology and Therapeutics, 2011. 89(2): p. 259-67.

150. Rowland Yeo, K., et al., Prediction of time-dependent CYP3A4 drug-drug interactions by physiologically based pharmacokinetic modelling: impact of inactivation parameters and enzyme turnover. European Journal of Pharmaceutical Sciences, 2011. 43(3): p. 160-73. 
151. Grime, K.H., et al., Mechanism-based inhibition of cytochrome P450 enzymes: an evaluation of early decision making in vitro approaches and drug-drug interaction prediction methods. European Journal of Pharmaceutical Sciences, 2009. 36(2-3): p. 175-91.

152. Gentry, P.R., et al., An approach for the quantitative consideration of genetic polymorphism data in chemical risk assessment: examples with warfarin and parathion. Toxicological Sciences, 2002. 70(1): p. 120-39.

153. Kusama, M., et al., Prediction of the effects of genetic polymorphism on the pharmacokinetics of CYP2C9 substrates from in vitro data. Pharmaceutical Research, 2009. 26(4): p. 822-35.

154. Teeguarden, J.G., et al., A PBPK model for evaluating the impact of aldehyde dehydrogenase polymorphisms on comparative rat and human nasal tissue acetaldehyde dosimetry. Inhalation Toxicology, 2008. 20(4): p. 375-90.

155. Baxter, L.T., et al., Biodistribution of monoclonal antibodies: scale-up from mouse to human using a physiologically based pharmacokinetic model. Cancer Research, 1995. 55(20): p. 4611-22.

156. Baxter, L.T., et al., Physiologically based pharmacokinetic model for specific and nonspecific monoclonal antibodies and fragments in normal tissues and human tumor xenografts in nude mice. Cancer Research, 1994. 54(6): p. 1517-28.

157. Puchalski, T., et al., Pharmacokinetic and pharmacodynamic modeling of an antiinterleukin-6 chimeric monoclonal antibody (siltuximab) in patients with metastatic renal cell carcinoma. Clinical Cancer Research, 2010. 16(5): p. 165261.

158. Davda, J.P., et al., A physiologically based pharmacokinetic (PBPK) model to characterize and predict the disposition of monoclonal antibody CC49 and its single chain Fv constructs. International Immunopharmacology, 2008. 8(3): p. 401-13.

159. Kletting, P., et al., Improving anti-CD45 antibody radioimmunotherapy using a physiologically based pharmacokinetic model. Journal of Nuclear Medicine, 2009. 50(2): p. 296-302.

160. Urva, S.R., et al., Physiologically based pharmacokinetic model for T84.66: a monoclonal anti-CEA antibody. Journal of Pharmaceutical Sciences, 2010. 99(3): p. $1582-600$.

161. Boswell, C.A., et al., Effects of anti-VEGF on predicted antibody biodistribution: roles of vascular volume, interstitial volume, and blood flow. PLoS ONE, 2011. 6(3): p. e17874. 
162. Friedrich, S.W., et al., Antibody-directed effector cell therapy of tumors: analysis and optimization using a physiologically based pharmacokinetic model.

Neoplasia, 2002. 4(5): p. 449-63.

163. Kletting, P., et al., Radioimmunotherapy with anti-CD66 antibody: improving the biodistribution using a physiologically based pharmacokinetic model. Journal of Nuclear Medicine, 2010. 51(3): p. 484-91.

164. Fallon, M.S., et al., A physiologically-based pharmacokinetic model of drug detoxification by nanoparticles. Journal of Pharmacokinetics and Pharmacodynamics, 2004. 31(5): p. 381-400.

165. Pery, A.R., et al., Development of a physiologically based kinetic model for 99mtechnetium-labelled carbon nanoparticles inhaled by humans. Inhalation Toxicology, 2009. 21(13): p. 1099-107.

166. Yang, R.S., et al., Pharmacokinetics and physiologically-based pharmacokinetic modeling of nanoparticles. Journal of Nanoscience and Nanotechnology, 2010. 10(12): p. 8482-90.

167. Li, M., et al., Physiologically based pharmacokinetic modeling of nanoparticles. ACS Nano, 2010. 4(11): p. 6303-17.

168. Xu, L., et al., Physiologically-based pharmacokinetics and molecular pharmacodynamics of 17-(allylamino)-17-demethoxygeldanamycin and its active metabolite in tumor-bearing mice. Journal of Pharmacokinetics and Pharmacodynamics, 2003. 30(3): p. 185-219.

169. Clewell, H.J., 3rd, et al., Development of a physiologically based pharmacokinetic model of isopropanol and its metabolite acetone. Toxicological Sciences, 2001. 63(2): p. 160-72.

170. Shah, D.K., et al., Physiologically based pharmacokinetic model for topotecan in mice. Journal of Pharmacokinetics and Pharmacodynamics, 2011. 38(1): p. 12142.

171. Nestorov, I., Modelling and simulation of variability and uncertainty in toxicokinetics and pharmacokinetics. Toxicology Letters, 2001. 120(1-3): p. 41120.

172. Jones, H.M., et al., A novel strategy for physiologically based predictions of human pharmacokinetics. Clinical Pharmacokinetics, 2006. 45(5): p. 511-42.

173. Pang, K.S., et al., Physiologically-based pharmacokinetic modeling for absorption, transport, metabolism and excretion. Journal of Pharmacokinetics and Pharmacodynamics, 2010. 37(6): p. 591-615. 
174. Jones, H.M., et al., Simulation of human intravenous and oral pharmacokinetics of 21 diverse compounds using physiologically based pharmacokinetic modelling. Clinical Pharmacokinetics, 2011. 50(5): p. 331-47.

175. Wishart, D.S., Improving early drug discovery through ADME modelling: an overview. Drugs in R\&D, 2007. 8(6): p. 349-62.

176. Leahy, D.E., Integrating in vitro ADMET data through generic physiologically based pharmacokinetic models. Expert Opinion on Drug Metabolism \& Toxicology, 2006. 2(4): p. 619-28.

177. Cai, H., et al., Evaluation of an integrated in vitro-in silico PBPK (physiologically based pharmacokinetic) model to provide estimates of human bioavailability. International Journal of Pharmaceutics, 2006. 308(1-2): p. 133-9.

178. De Buck, S.S., et al., Prediction of human pharmacokinetics using physiologically based modeling: a retrospective analysis of 26 clinically tested drugs. Drug Metabolism and Disposition, 2007. 35(10): p. 1766-80.

179. De Buck, S.S., et al., The prediction of drug metabolism, tissue distribution, and bioavailability of 50 structurally diverse compounds in rat using mechanismbased absorption, distribution, and metabolism prediction tools. Drug Metabolism and Disposition, 2007. 35(4): p. 649-59.

180. Theil, F.P., et al., Utility of physiologically based pharmacokinetic models to drug development and rational drug discovery candidate selection. Toxicology Letters, 2003. 138(1-2): p. 29-49.

181. Fang, L., et al., Predictive physiologically based pharmacokinetic model for antibody-directed enzyme prodrug therapy. Drug Metabolism and Disposition, 2008. 36(6): p. 1153-65.

182. Ferl, G.Z., et al., A two-tiered physiologically based model for dually labeled single-chain Fv-Fc antibody fragments. Molecular Cancer Therapeutics, 2006. 5(6): p. 1550-8.

183. Ferl, G.Z., et al., A predictive model of therapeutic monoclonal antibody dynamics and regulation by the neonatal $F c$ receptor ( $F c R n)$. Annals of Biomedical Engineering, 2005. 33(11): p. 1640-52.

184. Qin, S., et al., An imaging-driven model for liposomal stability and circulation. Molecular Pharmaceutics, 2010. 7(1): p. 12-21.

185. Barboriak, D.P., et al., Comparison of three physiologically-based pharmacokinetic models for the prediction of contrast agent distribution measured by dynamic MR imaging. Journal of Magnetic Resonance Imaging : JMRI, 2008. 27(6): p. 1388-98. 
186. Mescam, M., et al., A physiologically based pharmacokinetic model of vascularextravascular exchanges during liver carcinogenesis: application to MRI contrast agents. Contrast Media \& Molecular Imaging, 2007. 2(5): p. 215-28.

187. Armitage, P., et al., Extracting and visualizing physiological parameters using dynamic contrast-enhanced magnetic resonance imaging of the breast. Medical Image Analysis, 2005. 9(4): p. 315-29.

188. Zhu, H., et al., Tumor pretargeting for radioimmunodetection and radioimmunotherapy. Journal of Nuclear Medicine, 1998. 39(1): p. 65-76.

189. Zhu, H., et al., Potential and limitations of radioimmunodetection and radioimmunotherapy with monoclonal antibodies. Journal of Nuclear Medicine, 1997. 38(5): p. 731-41.

190. Hudachek, S.F., et al., Customized in silico population mimics actual population in docetaxel population pharmacokinetic analysis. Journal of Pharmaceutical Sciences, 2011. 100(3): p. 1156-66.

191. Bradshaw-Pierce, E.L., et al., Pharmacokinetic-directed dosing of vandetanib and docetaxel in a mouse model of human squamous cell carcinoma. Molecular Cancer Therapeutics, 2008. 7(9): p. 3006-17.

192. Wang, S., et al., Preclinical pharmacokinetic/pharmacodynamic models of gefitinib and the design of equivalent dosing regimens in EGFR wild-type and mutant tumor models. Molecular Cancer Therapeutics, 2008. 7(2): p. 407-17.

193. Wang, S., et al., Demonstration of the equivalent pharmacokinetic/pharmacodynamic dosing strategy in a multiple-dose study of gefitinib. Molecular Cancer Therapeutics, 2009. 8(6): p. 1438-47.

194. Zhou, Q., et al., The pharmacokinetic/pharmacodynamic pipeline: translating anticancer drug pharmacology to the clinic. The AAPS Journal, 2011. 13(1): p. 111-20.

195. Edginton, A.N., et al., Defining the role of macrophages in local moxifloxacin tissue concentrations using biopsy data and whole-body physiologically based pharmacokinetic modelling. Clinical Pharmacokinetics, 2009. 48(3): p. 181-7.

196. Tsukamoto, Y., et al., A physiologically based pharmacokinetic analysis of capecitabine, a triple prodrug of 5-FU, in humans: the mechanism for tumorselective accumulation of 5-FU. Pharmaceutical Research, 2001. 18(8): p. 1190202. 
197. Tsukamoto, Y., et al., Investigation of 5-FU disposition after oral administration of capecitabine, a triple-prodrug of 5-FU, using a physiologically based pharmacokinetic model in a human cancer xenograft model: comparison of the simulated 5-FU exposures in the tumour tissue between human and xenograft model. Biopharmaceutics \& Drug Disposition, 2001. 22(1): p. 1-14.

198. Gallo, J.M., et al., Pharmacokinetic model-predicted anticancer drug concentrations in human tumors. Clinical Cancer Research, 2004. 10(23): p. 8048-58.

199. Zhou, Q., et al., Predicting human tumor drug concentrations from a preclinical pharmacokinetic model of temozolomide brain disposition. Clinical Cancer Research, 2007. 13(14): p. 4271-9.

200. Zager, M.G., et al., A delayed nonlinear PBPK model for genistein dosimetry in rats. Bulletin of Mathematical Biology, 2007. 69(1): p. 93-117.

201. Schlosser, P.M., et al., Physiologically-based pharmacokinetic modeling of genistein in rats, Part I: Model development. Risk Analysis, 2006. 26(2): p. $483-$ 500 .

202. Li, J., et al., The effect of malignant effusions on methotrexate disposition. Cancer Chemotherapy and Pharmacology, 2002. 50(5): p. 373-82.

203. Devineni, D., et al., In vivo microdialysis to characterize drug transport in brain tumors: analysis of methotrexate uptake in rat glioma-2 (RG-2)-bearing rats. Cancer Chemotherapy and Pharmacology, 1996. 38(6): p. 499-507.

204. Bradshaw-Pierce, E.L., et al., A physiologically based pharmacokinetic model of docetaxel disposition: from mouse to man. Clinical Cancer Research, 2007. 13(9): p. 2768-76.

205. Jepson, G.W., et al., A partition coefficient determination method for nonvolatile chemicals in biological tissues. Fundamental and Applied Toxicology 1994. 22(4): p. 519-24.

206. Sung, J.H., et al., A combined pharmacokinetic-pharmacodynamic (PK-PD) model for tumor growth in the rat with UFT administration. Journal of Pharmaceutical Sciences, 2009. 98(5): p. 1885-904.

207. Poulin, P., et al., Prediction of pharmacokinetics prior to in vivo studies. 1. Mechanism-based prediction of volume of distribution. Journal of Pharmaceutical Sciences, 2002. 91(1): p. 129-56.

208. Poulin, P., et al., Prediction of pharmacokinetics prior to in vivo studies. II. Generic physiologically based pharmacokinetic models of drug disposition. Journal of Pharmaceutical Sciences, 2002. 91(5): p. 1358-70. 
209. Tweddle, D.A., et al., Evidence for the development of p53 mutations after cytotoxic therapy in a neuroblastoma cell line. Cancer Research, 2001. 61(1): p. $8-13$.

210. Vogan, K., et al., Absence of 53 gene mutations in primary neuroblastomas. Cancer Research, 1993. 53(21): p. 5269-73.

211. Tweddle, D.A., et al., The p53 pathway and its inactivation in neuroblastoma. Cancer Letters, 2003. 197(1-2): p. 93-8.

212. Zhang, F., et al., MDM2 antagonist nutlin-3a reverses mitoxantrone resistance by inhibiting breast cancer resistance protein mediated drug transport. Biochemical Pharmacology, 2011. 82(1): p. 24-34.

213. Zhang, F., et al., Whole-body physiologically based pharmacokinetic model for nutlin-3a in mice after intravenous and oral administration. Drug Metabolism and Disposition, 2011.39(1): p. 15-21.

214. LaRusch, G.A., et al., Nutlin3 blocks vascular endothelial growth factor induction by preventing the interaction between hypoxia inducible factor lalpha and Hdm2. Cancer Research, 2007. 67(2): p. 450-4.

215. Park, J.R., et al., Neuroblastoma: biology, prognosis, and treatment. Pediatric Clinics of North America, 2008. 55(1): p. 97-120, x.

216. Goodman, M.T., et al., Sympathetic nervous system tumors. Accessed Sep 6, 2011, Available from: http://seer.cancer.gov/publications/childhood/ sympathetic.pdf.

217. Garaventa, A., et al., High risk neuroblastoma: a persistent therapeutic challenge. Pediatric Blood \& Cancer, 2008. 51(6): p. 722-3.

218. Momand, J., et al., MDM2--master regulator of the 553 tumor suppressor protein. Gene, 2000. 242(1-2): p. 15-29.

219. Corvi, R., et al., Non-syntenic amplification of MDM2 and MYCN in human neuroblastoma. Oncogene, 1995. 10(6): p. 1081-6.

220. Chen, L., et al., p53 is nuclear and functional in both undifferentiated and differentiated neuroblastoma. Cell Cycle, 2007. 6(21): p. 2685-96.

221. Barbieri, E., et al., MDM2 inhibition sensitizes neuroblastoma to chemotherapyinduced apoptotic cell death. Molecular Cancer Therapeutics, 2006. 5(9): p. 235865 .

222. Van Maerken, T., et al., Small-molecule MDM2 antagonists as a new therapy concept for neuroblastoma. Cancer Research, 2006. 66(19): p. 9646-55. 
223. Peirce, S.K., et al., The MDM2 antagonist nutlin-3 sensitizes p53-null neuroblastoma cells to doxorubicin via E2F1 and TAp73. International Journal of Oncology, 2009. 34(5): p. 1395-402.

224. Peirce, S.K., et al., High level MycN expression in non-MYCN amplified neuroblastoma is induced by the combination treatment nutlin-3 and doxorubicin and enhances chemosensitivity. Oncology Reports, 2009. 22(6): p. 1443-9.

225. D'Argenio, D.Z., et al., ADAPT 5 User's Guide:

Pharmacokinetic/pharmacodynamic systems analysis software 2009, Los Angeles: Biomedical Simulations Resource.

226. Chou, T.C., Theoretical basis, experimental design, and computerized simulation of synergism and antagonism in drug combination studies. Pharmacological Reviews, 2006. 58(3): p. 621-81.

227. de Lange, J., et al., Synergistic growth inhibition based on small-molecule p53 activation as treatment for intraocular melanoma. Oncogene, 2011. Accessed Sep 6, 2011, Available from: http://dx.doi.org/10.1038/onc.2011.309.

228. Michaelis, M., et al., Reversal of P-glycoprotein-mediated multidrug resistance by the murine double minute 2 antagonist nutlin-3. Cancer Research, 2009. 69(2): $\mathrm{p}$. 416-21.

229. Secchiero, P., et al., Antiangiogenic activity of the MDM2 antagonist nutlin-3. Circulation Research, 2007. 100(1): p. 61-9.

230. Binder, B.R., A novel application for murine double minute 2 antagonists: the $p 53$ tumor suppressor network also controls angiogenesis. Circulation Research, 2007. 100(1): p. 13-4.

231. Eckford, P.D. , et al., ABC efflux pump-based resistance to chemotherapy drugs. Chemical Reviews, 2009. 109(7): p. 2989-3011.

232. Wierdl, M., et al., Carboxylesterase-mediated sensitization of human tumor cells to CPT-11 cannot override ABCG2-mediated drug resistance. Molecular Pharmacology, 2003. 64(2): p. 279-88.

233. Bai, F., et al., Determination of nutlin-3a in murine plasma by liquid chromatography electrospray ionization tandem mass spectrometry (LC-ESI$M S / M S)$. Journal of Pharmaceutical and Biomedical Analysis, 2009. 51(4): p. 915-20.

234. Huang, S.-M., et al., Graft guidance for industry drug interaction studies — study design, data analysis, and implications for dosing and labeling. 2006, Accessed Sep 6, 2011, Available from: http://www.fda.gov/downloads/Drugs/ GuidanceComplianceRegulatoryInformation/Guidances/ucm072101.pdf. 
235. Matsson, P., et al., A global drug inhibition pattern for the human ATP-binding cassette transporter breast cancer resistance protein (ABCG2). The Journal of Pharmacology and Experimental Therapeutics, 2007. 323(1): p. 19-30.

236. Doyle, L.A., et al., A multidrug resistance transporter from human MCF-7 breast cancer cells. Proceedings of the National Academy of Sciences of the United States of America, 1998. 95(26): p. 15665-70.

237. Polgar, O., et al., ABCG2: structure, function and role in drug response. Expert Opinion on Drug Metabolism \& Toxicology, 2008. 4(1): p. 1-15.

238. Mao, Q., et al., Role of the breast cancer resistance protein (ABCG2) in drug transport. The AAPS Journal, 2005. 7(1): p. E118-33.

239. Shiozawa, K., et al., Preclinical studies of vorinostat (suberoylanilide hydroxamic acid) combined with cytosine arabinoside and etoposide for treatment of acute leukemias. Clinical Cancer Research, 2009. 15(5): p. 1698-707.

240. Voigt, W., et al., Schedule-dependent antagonism of gemcitabine and cisplatin in human anaplastic thyroid cancer cell lines. Clininal Cancer Research, 2000. 6(5): p. 2087-93.

241. Mogi, M., et al., Akt signaling regulates side population cell phenotype via Bcrp1 translocation. The Journal of Biological Chemistry, 2003. 278(40): p. 39068-75.

242. Kruijtzer, C.M., et al., Increased oral bioavailability of topotecan in combination with the breast cancer resistance protein and P-glycoprotein inhibitor GF120918. Journal of Clinical Oncology : Official Journal of the American Society of Clinical Oncology, 2002. 20(13): p. 2943-50.

243. Stewart, C.F., et al., Gefitinib enhances the antitumor activity and oral bioavailability of irinotecan in mice. Cancer Research, 2004. 64(20): p. 7491-9.

244. Furman, W.L., et al., Tyrosine kinase inhibitor enhances the bioavailability of oral irinotecan in pediatric patients with refractory solid tumors. Journal of Clinical Oncology : Official Journal of the American Society of Clinical Oncology, 2009. 27(27): p. 4599-604.

245. Carcaboso, A.M., et al., Tyrosine kinase inhibitor gefitinib enhances topotecan penetration of gliomas. Cancer Research, 2010. 70(11): p. 4499-508.

246. Hollstein, M., et al., P53 mutations in human cancers. Science, 1991. 253(5015): p. 49-53.

247. Shangary, S., et al., Targeting the MDM2-p53 interaction for cancer therapy. Clinical Cancer Research, 2008. 14(17): p. 5318-24. 
248. Klein, C., et al., Targeting the p53-MDM2 interaction to treat cancer. British Journal of Cancer, 2004. 91(8): p. 1415-9.

249. Miyachi, M., et al., Restoration of 553 pathway by nutlin-3 induces cell cycle arrest and apoptosis in human rhabdomyosarcoma cells. Clinical Cancer Research, 2009. 15(12): p. 4077-84.

250. Elison, J.R., et al., Small molecule inhibition of HDM2 leads to p53-mediated cell death in retinoblastoma cells. Archives of Ophthalmology, 2006. 124(9): p. 126975.

251. Wan, H., et al., High-throughput screening of protein binding by equilibrium dialysis combined with liquid chromatography and mass spectrometry. Journal of Chromatography. A, 2006. 1102(1-2): p. 125-34.

252. Kariv, I., et al., Development of a high throughput equilibrium dialysis method. Journal of Pharmaceutical Sciences, 2001. 90(5): p. 580-7.

253. $\mathrm{Xu}, \mathrm{X}$., et al., Nonlinear protein binding and enzyme heterogeneity: effects on hepatic drug removal. Journal of Pharmacokinetics and Biopharmaceutics, 1993. 21(1): p. 43-74.

254. Brown, R.P., et al., Physiological parameter values for physiologically based pharmacokinetic models. Toxicology and Industrial Health, 1997. 13(4): p. 40784.

255. Wright, W.S., et al., Attenuation of diabetes-induced retinal vasoconstriction by a thromboxane receptor antagonist. Experimental Eye Research, 2009. 88(1): p. 106-12.

256. Barbieri, A., et al., Simultaneous determination of low levels of methotrexate and cyclophosphamide in human urine by micro liquid chromatography/electrospray ionization tandem mass spectrometry. Rapid Communications in Mass Spectrometry : RCM, 2006. 20(12): p. 1889-93.

257. Lee, Y.M., et al., Nutlin-3, a Hdm2 antagonist, inhibits tumor adaptation to hypoxia by stimulating the FIH-mediated inactivation of HIF-1 \{alpha\}. Carcinogenesis, 2009. 30(10): p. 1768-75.

258. Laurie, N.A., et al., Topotecan combination chemotherapy in two new rodent models of retinoblastoma. Clinical Cancer Research, 2005. 11(20): p. 7569-78.

259. Shields, C.L., Forget-me-nots in the care of children with retinoblastoma. Seminars in Ophthalmology, 2008. 23(5): p. 324-34.

260. Amemiya, T., et al., Vitreous seeds in retinoblastoma, clinical significance and ultrastructure. Albrecht Von Graefe's Archive for Clinical and Experimental Ophthalmology, 1979. 211(3): p. 205-13. 
261. Shires, T.K., et al., Protein levels in the vitreous of rats with streptozotocininduced diabetes mellitus. Brain Research Bulletin, 1993. 30(1-2): p. 85-90.

262. Kamb, A., et al., Why is cancer drug discovery so difficult? Nature Reviews. Drug Discovery, 2007. 6(2): p. 115-20.

263. Adams, C.P., et al., Spending on new drug development. Healthcare Economist, 2010. 19(2): p. 130-41.

264. DiMasi, J.A., et al., The price of innovation: new estimates of drug development costs. Journal of Health Economics, 2003. 22(2): p. 151-85.

265. DiMasi, J.A., et al., The cost of biopharmaceutical $R \& D$ : is biotech different? Managerial and Decision Economics, 2007. 28(4-5): p. 469-79. 


\section{VITA}

Fan Zhang was born in China in 1978. In 1996, Fan Zhang enrolled in Xi'an Jiaotong University, major in Clinical Medicine. In 2001, Fan received the Bachelor of Medicine degree with honor. In 2004, she received the Master of Science degree in Microbiology from the same University. She enrolled in the Ph.D. program in the Department of Pharmaceutical Sciences at the University of Tennessee Health Science Center in 2006 and received Ph.D. degree in 2011. Her dissertation research was conducted at St. Jude Children's Research Hospital in Dr. Clinton Stewart's lab. She is a member of the Rho Chi Honor Society. 